University of Tennessee Health Science Center UTHSC Digital Commons

\title{
$5-2009$
}

\section{The Role of Inflammation in the Early Radiation Response}

Christy Marie Wilson

University of Tennessee Health Science Center

Follow this and additional works at: https://dc.uthsc.edu/dissertations

Part of the Neoplasms Commons, and the Therapeutics Commons

\section{Recommended Citation}

Wilson, Christy Marie, "The Role of Inflammation in the Early Radiation Response" (2009). Theses and Dissertations (ETD). Paper 304. http://dx.doi.org/10.21007/etd.cghs.2009.0353.

This Dissertation is brought to you for free and open access by the College of Graduate Health Sciences at UTHSC Digital Commons. It has been accepted for inclusion in Theses and Dissertations (ETD) by an authorized administrator of UTHSC Digital Commons. For more information, please contact jwelch30@uthsc.edu. 


\title{
The Role of Inflammation in the Early Radiation Response
}

\begin{abstract}
Radiation is one of the principal treatments for adults and children with brain tumors, and is one of the oldest established treatments for tumors of all types. Currently, the limiting factor for the use of radiation is the effect on normal tissue adjacent to the tumor. Toxicity, including early and late effects from radiation, limits the dose administered to the tumor and reduces the probability of cure. This work has three aims in its attempt to understand and limit early radiation damage: characterize the role of the inflammatory molecules tumor necrosis factor alpha (TNF) and intracellular adhesion molecule-1 (ICAM-1) in the radiation response; evaluate the role of a novel anti-inflammatory agent as an interventional therapy to limit the radiation-induced inflammatory response; begin to characterize the acute damage following radiation using the shift in the gene expression.
\end{abstract}

Fluorescence intravital microscopy and a mouse cranial window model were used to quantitatively measure: permeability of the blood-brain barrier, leukocyte adhesion, and changes in vessel diameter following a single dose of 20-Gy localized cranial irradiation. Immunohistochemistry and immunofluorescence staining were used to evaluate the number of activated astrocytes and the protein expression of TNF and ICAM-1. Antibodies to TNF and ICAM-1 were administered to investigate their role in the early radiation response. In addition, a novel anti-inflammatory agent, KZ-41, which is thought to work through NF-KB was evaluated as a non-specific inhibitor to the early radiation response. Microarray analysis was used to characterize the molecular shift that occurs in the brain at 2-hrs after 20-Gy radiation.

Our results show that the inflammatory molecules TNF and ICAM- 1 are involved in the early radiation response. Radiation induces an increase in permeability of the BBB and in the number of adhering leukocytes at 24- and 48-hrs post-irradiation. It also causes a decrease in the average diameter of arterioles at 48-hrs post-irradiation. Immunohistochemistry showed a significant increase in the number of activated astrocytes at 24-and 48-hrs post-irradiation, while immunofluorescence verified the expression of TNF and ICAM-1 protein following radiation. When TNF expression was inhibited via specific antibodies, all of the radiation-induced effects were abrogated. When ICAM- 1 was inhibited via specific antibodies, most of the radiation-induced effects were abrogated. Treatment with ICAM-1 mAb in conjunction with radiation, completely inhibited the radiation-induced vascular effects (BBB permeability, leukocyte adhesion, and arteriole diameter changes), but did not inhibit activated astrocytes, however it did significantly reduce them compared to radiation alone.

KZ-41 was evaluated as a potential agent for interventional therapy aimed at reducing the radiationinduced inflammatory response. Using our radiation model, KZ-41 was found to protect the vasculature in a manner similar to the specific antibodies to TNF or ICAM-1, but was unable to prevent the radiationinduced astrocytic response. Permeability of the BBB and leukocyte adhesion was inhibited, while there was no observed change in arteriole diameter at any time point. Immunofluorescence showed that treatment with KZ-41 limited the radiation-induced expression of ICAM-1 protein, but did not alter the expression of TNF protein. In addition, the number of activated astrocytes, following treatment with $\mathrm{KZ}-41$, was significantly higher than in radiation only animals.

Microarray analysis was used to investigate the shift in the genetic profile of the brain following radiation. It was seen that, 2-hrs following irradiation, there is: a paracrine response exists that is primarily initiated by chemotactic cytokines through the cytokine-cytokine receptor interaction pathway; vascular andparenchymal damage whose response can be distinguished when gene function is considered; and an alteration in genes known to be related to neurological/neurodegenerative diseases.

Our overall aim is to elucidate the role of inflammation in the acute radiation response and its effects on 
the microvasculature of the brain. Understanding this role will allow for the development of treatment strategies to limit the acute radiation damage which we believe will lead to a reduction in the long-term side effects of radiation.

\section{Document Type}

Dissertation

Degree Name

Doctor of Philosophy (PhD)

\section{Program}

Biomedical Engineering and Imaging

\section{Research Advisor}

M. Waleed Gaber, Ph.D.

\section{Keywords}

Radiation, Inflammation, Blood-Brain Barrier, Gene Expression, Tumor Necrosis Factor, Vasculature

\section{Subject Categories}

Analytical, Diagnostic and Therapeutic Techniques and Equipment | Diseases | Medicine and Health

Sciences | Neoplasms | Therapeutics 
THE ROLE OF INFLAMMATION IN THE EARLY RADIATION RESPONSE

\author{
A Dissertation \\ Presented for \\ The Graduate Studies Council \\ The University of Tennessee \\ Health Science Center
}
In Partial Fulfillment
Of the Requirements for the Degree
Doctor of Philosophy
In the Joint Graduate Program in Biomedical Engineering and Imaging
From The University of Tennessee
and
The University of Memphis

By

Christy Marie Wilson

May 2009 
Copyright (C) 2009

by

Christy Marie Wilson

All rights reserved 


\section{Acknowledgements}

I would like to thank my research advisor Dr M Waleed Gaber for all that he has done for me during my studies. He was always involved with my work and provided constant guidance and encouragement. I would also like to thank Dr Omaima Sabek for all of the help, support, and encouragement she gave me along the way. I would like to think $\mathrm{Dr}$ Thomas Merchant for all of his wonderful insight, encouragement, and advice. I would also like to acknowledge my committee members: Dr Frank DiBianca, Dr Chris Waters, and Dr Lisa Jennings for their help and suggestions. I would like to thank Ms. Janice Zawaski for everything that she did to help me in the lab over the years, as it would have been very hard without her and much less enjoyable. I would also like to thank Dr Gary Keyes, Dr Ed Schneider, and Dr Richard Peppler for all of their support and encouragement over the years. I would like to express my gratitude and thanks to my parents for all of their love and support throughout my entire education. Last but not least, I would like to thank my wonderful and loving husband for everything that he has done to help me, I couldn't have done it without him.

I would like to thank Dr Ryan Yates and Dr Duane Miller for providing the KZ-41 used in this work, and I would like to acknowledge the American Heart Association (0365250B) and the UT Alma and Hal Reagan Fellowship for the study of the causes and cures for cancer for funding this research. 


\section{Abstract}

Radiation is one of the principal treatments for adults and children with brain tumors, and is one of the oldest established treatments for tumors of all types. Currently, the limiting factor for the use of radiation is the effect on normal tissue adjacent to the tumor. Toxicity, including early and late effects from radiation, limits the dose administered to the tumor and reduces the probability of cure. This work has three aims in its attempt to understand and limit early radiation damage: characterize the role of the inflammatory molecules tumor necrosis factor alpha (TNF) and intracellular adhesion molecule-1 (ICAM-1) in the radiation response; evaluate the role of a novel anti-inflammatory agent as an interventional therapy to limit the radiation-induced inflammatory response; begin to characterize the acute damage following radiation using the shift in the gene expression.

Fluorescence intravital microscopy and a mouse cranial window model were used to quantitatively measure: permeability of the blood-brain barrier, leukocyte adhesion, and changes in vessel diameter following a single dose of 20-Gy localized cranial irradiation. Immunohistochemistry and immunofluorescence staining were used to evaluate the number of activated astrocytes and the protein expression of TNF and ICAM-1. Antibodies to TNF and ICAM-1 were administered to investigate their role in the early radiation response. In addition, a novel anti-inflammatory agent, KZ-41, which is thought to work through NF$\kappa \mathrm{B}$ was evaluated as a non-specific inhibitor to the early radiation response. Microarray analysis was used to characterize the molecular shift that occurs in the brain at 2-hrs after 20-Gy radiation.

Our results show that the inflammatory molecules TNF and ICAM-1 are involved in the early radiation response. Radiation induces an increase in permeability of the BBB and in the number of adhering leukocytes at 24- and 48-hrs post-irradiation. It also causes a decrease in the average diameter of arterioles at 48-hrs post-irradiation. Immunohistochemistry showed a significant increase in the number of activated astrocytes at 24and 48-hrs post-irradiation, while immunofluorescence verified the expression of TNF and ICAM-1 protein following radiation. When TNF expression was inhibited via specific antibodies, all of the radiation-induced effects were abrogated. When ICAM-1 was inhibited via specific antibodies, most of the radiation-induced effects were abrogated. Treatment with ICAM-1 mAb in conjunction with radiation, completely inhibited the radiation-induced vascular effects (BBB permeability, leukocyte adhesion, and arteriole diameter changes), but did not inhibit activated astrocytes, however it did significantly reduce them compared to radiation alone.

$\mathrm{KZ}-41$ was evaluated as a potential agent for interventional therapy aimed at reducing the radiation-induced inflammatory response. Using our radiation model, KZ-41 was found to protect the vasculature in a manner similar to the specific antibodies to TNF or ICAM-1, but was unable to prevent the radiation-induced astrocytic response. Permeability of the BBB and leukocyte adhesion was inhibited, while there was no observed change in arteriole diameter at any time point. Immunofluorescence showed that treatment with KZ-41 limited the radiation-induced expression of ICAM-1 protein, but did not alter the expression 
of TNF protein. In addition, the number of activated astrocytes, following treatment with $\mathrm{KZ}-41$, was significantly higher than in radiation only animals.

Microarray analysis was used to investigate the shift in the genetic profile of the brain following radiation. It was seen that, 2 -hrs following irradiation, there is: a paracrine response exists that is primarily initiated by chemotactic cytokines through the cytokinecytokine receptor interaction pathway; vascular and parenchymal damage whose response can be distinguished when gene function is considered; and an alteration in genes known to be related to neurological/neurodegenerative diseases.

Our overall aim is to elucidate the role of inflammation in the acute radiation response and its effects on the microvasculature of the brain. Understanding this role will allow for the development of treatment strategies to limit the acute radiation damage which we believe will lead to a reduction in the long-term side effects of radiation. 


\section{Table of Contents}

$\begin{array}{lll}\text { Chapter } 1 \text { Objectives } & 1\end{array}$

1.1 Hypotheses . . . . . . . . . . . . . . . . . . . . . 2

1.2 Specific aims . . . . . . . . . . . . . . . . . . 2

Chapter 2 Introduction $\quad 4$

2.1 Use of radiotherapy in the clinic to treat brain tumors . . . . . . . . . . . . 4

2.2 Side effects of radiotherapy and current treatments . . . . . . . . . . . . . 5

2.3 How radiation damages cells . . . . . . . . . . . . . . . . . . 7

2.4 Sensitivity to radiation damage . . . . . . . . . . . . . . . 9

2.5 The brain: components and response to radiotherapy . . . . . . . . . 11

2.6 Radiation-induced inflammation and current clinical treatments . . . . . . . 16

$2.7 \mathrm{KZ}-41 \ldots \ldots \ldots \ldots \ldots \ldots$

2.8 Gene expression . . . . . . . . . . . . . . . . . . . . . . . . . . . 21

Chapter 3 Materials and Methods 25

3.1 In vivo measurements . . . . . . . . . . . . . . . . . 25

3.1 .1 Animal model . . . . . . . . . . . . . . . . . . . 25

3.1 .2 Cranial window . . . . . . . . . . . . . . . . . 25

3.1 .3 Radiation treatment . . . . . . . . . . . . . . . 25

3.1 .4 Anti-inflammatory treatments . . . . . . . . . . . . . 27

3.1 .5 Recombinant TNF . . . . . . . . . . . . . . . . . . . . . . 27

3.1.6 Intravital microscopy and BBB permeability . . . . . . . . . . . . 27

3.1 .7 Leukocyte-endothelial interactions . . . . . . . . . . . . . . . . . 30

3.1 .8 Vessel diameter measurements . . . . . . . . . . . . . . . . . . . 30

3.1 .9 Statistics . . . . . . . . . . . . . . . . . . . . . 31

3.2 Histological measurements . . . . . . . . . . . . . . . . . . 33

3.2 .1 Tissue collection . . . . . . . . . . . . . . . . . . . . 33

3.2 .2 Immunohistological staining . . . . . . . . . . . . . . 33

3.2.3 Immunofluorescence staining . . . . . . . . . . . . . . 33

3.2 .4 Statistics . . . . . . . . . . . . . . . . . 33 
3.3 Microarray experiments $\ldots \ldots \ldots \ldots \ldots \ldots \ldots \ldots \ldots \ldots$

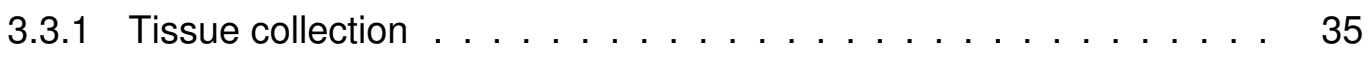

3.3.2 RNA preparation . . . . . . . . . . . . . . . 35

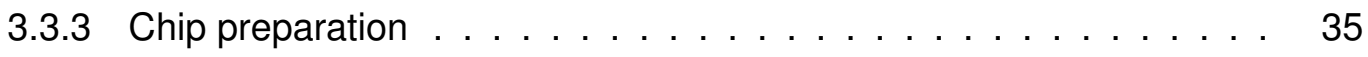

3.3.4 Chip analysis . . . . . . . . . . . . . . . . . . . 35

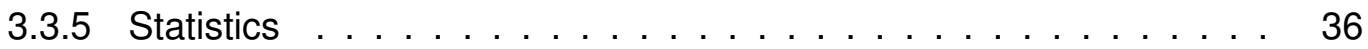

3.4 Serum cytokines $\ldots \ldots \ldots \ldots \ldots \ldots \ldots \ldots \ldots \ldots$

Chapter 4 Mechanism of Radiation-Induced Inflammation 37

4.1 Radiation-induced changes in the permeability of the BBB $\ldots \ldots$. . . . 37

4.2 Radiation-induced leukocyte adhesion in the brain . . . . . . . . . . . . 39

4.3 Changes in pial vessel diameters following cranial irradiation . . . . . . . 39

4.4 Correlation between BBB permeability and leukocyte adhesion . . . . . . 39

4.5 Immunohistochemistry . . . . . . . . . . . . . . . . . 43

4.6 Immunofluorescence . . . . . . . . . . . . . . . . . . 50

4.7 Effect of TNF on cerebral vasculature . . . . . . . . . . . . . 50

4.8 Discussion . . . . . . . . . . . . . . . . . 50

Chapter 5 KZ-41: An Interventional Therapy 58

5.1 Effect of KZ-41 on radiation-induced changes to the microvasculature . . . 59

5.2 Effect of KZ-41 on the radiation-induced astrocytic response . . . . . . . . 59

5.3 Inhibition of protein expression using KZ-41 . . . . . . . . . . . 66

5.4 Discussion ............................ 66

Chapter 6 Early Markers of Radiation-Induced Damage $\quad 70$

6.1 Microarray analysis . . . . . . . . . . . . . . . . . . 72

6.2 Paracrine response . . . . . . . . . . . . . . . . . . . 72

6.3 Vascular versus parenchymal . . . . . . . . . . . . . . . . . . 78

6.4 Early signs of neurostructural changes . . . . . . . . . . . . . . 78

6.5 Genes of interest . . . . . . . . . . . . . . . . . . . . . . . 83

6.6 Cytokine analysis . . . . . . . . . . . . . . . . . . 85

6.7 Discussion . . . . . . . . . . . . . . . . . . . . . . 85

$\begin{array}{lll}\text { Chapter } 7 & \text { Discussion } & 87\end{array}$

7.1 Radiation-induced vascular changes . . . . . . . . . . . . . . 87 
7.2 Histology . . . . . . . . . . . . . . . . . . 89

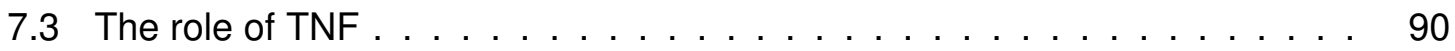

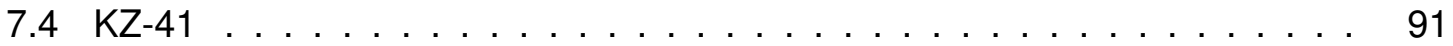

7.5 Early markers of radiation-induced damage $\ldots \ldots \ldots \ldots . \ldots . \ldots 92$

7.5 .1 Gene expression . . . . . . . . . . . . . . . . . . . . . 92

7.5 .2 Serum cytokines . . . . . . . . . . . . . . . . 93

Chapter 8 Conclusions and Future Work 94

8.1 Conclusions . . . . . . . . . . . . . . . . . . . . . . 94

8.2 Future Work . . . . . . . . . . . . . . . . . . . . . . . . . . 94

$\begin{array}{ll}\text { List of References } & 97\end{array}$

Appendix A Cranial Window Surgery Protocol 112

$\begin{array}{ll}\text { Appendix B Immunofluorescence Staining Protocol } & 114\end{array}$

$\begin{array}{ll}\text { Vita } & 115\end{array}$ 


\section{List of Tables}

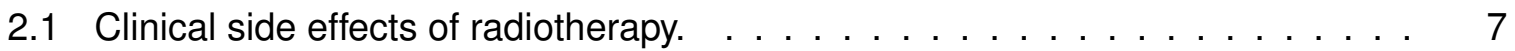

2.2 Classification of cell and tissue radiosensitivity. . . . . . . . . . . . . . . . 10

2.3 Radiobiologic side effects of radiotherapy. . . . . . . . . . . . . . . . . . . . . 14

6.1 Significant genes by function/pathway. . . . . . . . . . . . . . . . 73

6.2 Paracrine response genes. . . . . . . . . . . . . . . . . 76

6.3 Paracrine response pathways. . . . . . . . . . . . . . . . . 77

6.4 Genes related to the response of the vasculature. . . . . . . . . . . . . . 79

6.5 Genes related to the response of the parenchyma. . . . . . . . . . . . . . . . 80

6.6 Pathway classification of the genes related to the vascular response. . . . . . . 81

6.7 Pathway classification of the genes related to the parenchymal response. . . . 82

6.8 Modulated genes related to development and neurodegenerative diseases. . . 84

6.9 Serum cytokine results. . . . . . . . . . . . . . . . . . . 86

A.1 Artificial cerebral spinal fluid. . . . . . . . . . . . . . . . . . . . 113 


\section{List of Figures}

2.1 Radiation dose map. . . . . . . . . . . . . . . . . . . . . . 6

2.2 Late effects of radiation. . . . . . . . . . . . . . . . . . . . 6

2.3 Direct versus indirect radiation. . . . . . . . . . . . . . . . . 8

2.4 Cartoon depiction of the normal and damaged BBB. . . . . . . . . . . . . 13

2.5 Pathophysiology of radiation-induced damage. . . . . . . . . . . . . . . . 17

2.6 Radiation-induced cellular response. . . . . . . . . . . . . . . . . . . . . 18

2.7 Effect of $\mathrm{KZ}-41$ on vascular injury. . . . . . . . . . . . . . . . . . . . . 20

$2.8 \mathrm{KZ}-41$ inhibits leukopenia. . . . . . . . . . . . . . . . . . . . . . . 22

$2.9 \mathrm{KZ}-41$ prevents weight loss following total body irradiation. . . . . . . . . . . . 22

3.1 Picture of a mouse after placement of the cranial window. . . . . . . . . . . 26

3.2 Irradiation set-up. . . . . . . . . . . . . . . . . . . . 26

3.3 A picture of the intravital microscopy set-up. . . . . . . . . . . . . . . . 28

3.4 FITC-dextran 4.4kDa injection. . . . . . . . . . . . . . . . . . . . . 29

3.5 Permeability measurement. . . . . . . . . . . . . . . . . . . . 31

3.6 Vessel diameter measurement. . . . . . . . . . . . . . . . . . . . . . . 32

3.7 Regions used for histological analysis. . . . . . . . . . . . . . . . . . . . 34

4.1 Vascular permeability of the BBB following irradiation. . . . . . . . . . 38

4.2 Leukocyte adhesion following irradiation. . . . . . . . . . . . . . . . . 40

4.3 Representative images of radiation-induced leukocyte adhesion. . . . . . . . . 41

4.4 Radiation-induced changes in vessel diameter. . . . . . . . . . . . . . . . 42

4.5 Correlation between BBB permeability and leukocyte adhesion. . . . . . . . . . 44

4.6 Activated astrocyte counts in the cerebral cortex. . . . . . . . . . . . . . . . 47

4.7 GFAP staining for activated astrocytes. . . . . . . . . . . . . . . . . . . . . 48

4.8 Activated astrocyte counts in the dentate gyrus region. . . . . . . . . . . . . . 49

4.9 Immunofluorescence staining. . . . . . . . . . . . . . . . . . . . . . . 51

4.10 TNF induces changes in vessel diameter. . . . . . . . . . . . . . . . . . . 54

4.11 TNF induces leukocyte adhesion. . . . . . . . . . . . . . . . . . 55

4.12 TNF induces astrogliosis. . . . . . . . . . . . . . . . . . . . 56 
5.1 Vascular permeability of the BBB following radiotherapy following KZ-41 . . . 60

5.2 Leukocyte adhesion following radiotherapy can be inhibited by KZ-41 . . . . . 61

5.3 Representative images of radiation-induced leukocyte adhesion. . . . . . . . 62

5.4 Radiation-induced changes in vessel diameter. . . . . . . . . . . . . . . 63

5.5 Activated astrocyte counts in the cerebral cortex is exacerbated with KZ-41. . . 64

5.6 GFAP staining for activated astrocytes. . . . . . . . . . . . . . . . . . 65

5.7 Activated astrocyte counts in the dentate gyrus region following radiation and KZ-41. . . . . . . . . . . . . . . . . . . . . . 67

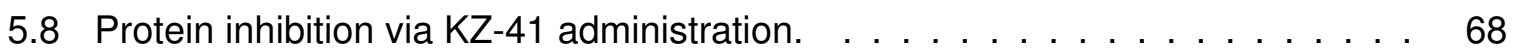

6.1 Schematic of the acute molecular responses following radiation. . . . . . . 71

6.2 Use of cytokine levels as a clinical biomarker. . . . . . . . . . . . . . . . . 86 


\section{List of Abbreviations}

$\begin{array}{ll}\text { Anti-ICAM-1 } & \text { Antibody to ICAM-1 } \\ \text { Anti-TNF } & \text { Antibody to TNF } \\ \text { APP } & \text { Amyloid Precursor Protein } \\ \text { BBB } & \text { Blood-Brain Barrier } \\ \text { CNS } & \text { Central Nervous System } \\ \text { DNA } & \text { Deoxyribonucleic Acid } \\ \text { FITC } & \text { Fluorescein Isothiocyanate } \\ \text { G-CSF } & \text { Granulocyte Colony-Stimulating Factor } \\ \text { GFAP } & \text { Glial Fibrillary Acidic Protein } \\ \text { Gy } & \text { Gray } \\ \text { HIF-1 } \alpha & \text { Hypoxia-Inducible Factor 1, Alpha Subunit } \\ \text { i.m. } & \text { Intramuscular Injection } \\ \text { i.p. } & \text { Intraperitoneal Injection } \\ \text { i.v. } & \text { Intravenous Injection } \\ \text { I-CAM-1 } & \text { Intracellular Adhesion Molecule-1 } \\ \text { IgG } & \text { Immunoglobulin G } \\ \text { IL-10 } & \text { Interleukin-10 } \\ \text { IL-6 } & \text { Interleukin-6 } \\ \text { kDa } & \text { Kilodalton } \\ \text { KEGG } & \text { Kyoto Encyclopedia of Genes and Genomes } \\ \text { mAb } & \text { Monoclonal Antibody } \\ \text { mRNA } & \text { Messenger Ribonucleic Acid } \\ \text { NF- } \kappa \text { B } & \text { Nuclear Factor- } \kappa \text { B } \\ \text { RNA } & \text { Ribonucleic Acid } \\ \text { ROI } & \text { Region of Interest } \\ \text { RT } & \text { Radiation Therapy } \\ \text { rTNF } & \text { Recombinant Tumor Necrosis Factor Alpha } \\ \text { TNF } & \text { Tumor Necrosis Factor Alpha } \\ \text { VEGF } & \text { Vascular Endothelial Growth Factor } \\ & \end{array}$




\section{Chapter 1}

\section{Objectives}

Radiation is one of the principal treatments for adults and children with brain tumors, and is one of the oldest established treatments for tumors of all types. Currently, the limiting factor for the use of radiation is the effect on normal tissue adjacent to the tumor. Toxicity, including early and late effects from radiation, limits the dose administered to the tumor and reduces the probability of cure. In the brain, toxicity arises from a combination of vascular and parenchymal damage and varies in incidence and severity relative to dose and volume. According to the Brain Tumor Progress Review Group, in order to overcome this normal tissue toxicity, scientists should aim to "delineate the molecular, cellular, and physiological processes leading to radiation-induced toxicity" [1]. Our overall hypothesis is that the early radiation-induced damage to the microvascular network in the brain is primarily responsible for the late effects observed in the normal tissue, and that by limiting the early damage, the late effects can be reduced or prevented. In addition, we hypothesize that the early damage to the microvasculature is due to the acute inflammatory response that is initiated following radiation and that understanding the role of inflammation is key to determining a way to reduce the side effects of radiation therapy and limit normal tissue damage.

Previously, our lab has shown that, in the brain and cremaster muscle, radiation increases vascular permeability and leukocyte rolling and adhesion. Changes in vascular permeability in the brain have been observed in a single dose (20-Gy) and fractionated dose (2-Gy/day for 4 weeks; 40-Gy total) radiation regimen. Our lab has also shown that there is an increase in the inflammatory molecules tumor necrosis factor-alpha (TNF) and intracellular adhesion molecule-1 (ICAM-1), at the mRNA level, two hours after a single dose and after the final fraction in the fractionated regimen. Others have confirmed the increase in ICAM-1 expression following cranial radiation, and have also shown an increase in interleukin-1 $\beta$. We have also shown that the expression of ICAM-1 can be abrogated following treatment with antibodies to ICAM-1, and that by abrogating ICAM-1 expression, permeability and leukocyte adhesion can be inhibited. This work demonstrated a relationship between the acute changes in the microvasculature and the inflammatory response that is initiated following radiation. The current work aims to further elucidate the role of the inflammatory response in the initiation and propagation of the acute microvasculature changes by evaluating the role of TNF. This work also evaluates a novel anti-inflammatory agent for use as a potential interventional therapy. In addition, this work evaluates the acute damage following radiation by characterizing the gene profile using microarray analysis. 


\subsection{Hypotheses}

1. Brain irradiation causes an acute response which if unchecked creates a chronic inflammatory microenvironment which amplifies radiation sequela. Treatment with anti-TNF or anti-ICAM-1 agents during the early phase arrests this inflammatory response and reduces radiation toxicity of the normal brain tissue.

2. $\mathrm{KZ}-41$, a derivative of quinic acid that shows anti-inflammatory effects through the NF- $\kappa$ B/ICAM-1 pathway, can be used to inhibit the inflammation-mediated acute radiation effects.

3. The gene profile of the acute damage following radiation can give an insight into the inflammatory, vascular, and parenchymal pathways that could be targeted for interventional therapy to abrogate radiation-induced late effects.

\subsection{Specific aims}

1. Characterize the acute damage to the blood-brain barrier, following a single dose of 20-Gy localized cranial irradiation using a mouse cranial window model and histological methods and evaluate the effect of treatment with anti-TNF or anti-ICAM-1 in conjunction with irradiation to abrogate inflammation-induced radiation damage.

Specific questions being investigated:

- What is the role of TNF and ICAM-1 in the acute changes observed following radiation?

- Does inhibiting TNF affect the levels of ICAM-1 and visa versa?

- Is there a correlation between the increase in vascular permeability and leukocyte adhesion? Does blocking either receptor, TNF or ICAM-1, disrupt this correlation?

2. Evaluate the effectiveness of $\mathrm{KZ}-41$ as an interventional therapy for acute radiation damage to the blood-brain barrier following a single dose of 20-Gy localized cranial irradiation and compare to the response observed from the direct inhibition of the inflammatory molecules TNF and ICAM-1 via antibodies.

Specific questions being investigated:

- Is KZ-41 able to inhibit the radiation-induced vascular changes known to be effected by TNF and ICAM-1?

- Does KZ-41 affect the levels of TNF or ICAM-1 following localized cranial radiation?

- If KZ-41 works through the ICAM-1 pathway only, does it decouple the vascular response from the astrocytic response? 
3. Investigate the acute inflammatory, vascular, parenchymal, and neurostructural changes in the brain following radiation damage by characterizing the gene response using $\mathrm{mi}-$ croarray gene expression analysis.

Specific questions being investigated:

- Is there a paracrine response that occurs?

- Can we distinguish between parenchymal and vascular damage?

- Are there early signs of neurostructural damage? 


\section{Chapter 2}

\section{Introduction}

\subsection{Use of radiotherapy in the clinic to treat brain tumors}

Each year approximately 50,000 people are diagnosed with a primary brain tumor and another 180,000 people develop brain metastases. The outcome of a patient diagnosed with a brain tumor depends on a variety of factors including: the location of the tumor, the type of tumor, age at time of diagnosis, and overall health [2]. The most common sequence of treatment for a brain tumor consists of surgical resection followed by adjuvant radiation therapy and chemotherapy when indicated. Gross removal of the tumor is often the best treatment; however, due to the intricate nature and complexity of the brain, surgery may not be feasible or is likely to be incomplete, necessitating the use of radiation therapy, chemotherapy or a combination of the two. Chemotherapy is used to treat brain tumors, but often fails due to a lack of sensitivity of the tumor to a given agent or because of the barriers (blood-brain barrier and blood-tumor barrier) in the brain, that limit therapeutic concentrations of a chemotherapeutic agent from reaching the tumor. Radiation is the most effective non-surgical therapy for brain tumors and is often the most effective adjuvant therapy after surgery. The effectiveness of radiation therapy is offset by its deleterious effects on normal tissues. Patients treated with radiotherapy risk neurologic, endocrine, and cognitive dysfunction resulting from damage to the microvasculature and parenchyma $[3,4]$. In recent years, there have been significant advances in the use of radiation as a treatment including hardware and software, aimed at improving tumor control and minimizing side effects. The advances include conformal, intensity-modulated and proton beam radiation therapy. All of these methods rely on 3D treatment planning concepts that have the goal of conforming dose to the tumor and minimizing dose to normal tissue. Hardware advances include the use of multi-leaf collimators to tailor the shape of the radiation field to a target volume, which has dramatically reduced the dose received by the normal tissue. The main advance in software has been the ability to incorporate the data from multiple imaging modalities (CT, MRI, and PET) into a single treatment planning system to aid in pretreatment evaluation and tumor delineation. All of these advances in the field of radiation therapy have raised the hope of more effective treatment; however, side effects continue to limit the value of radiotherapy in some patients.

There are two classes of brain tumors: primary and metastatic. Primary brain tumors are tumors that arise from the cells of the central nervous system (CNS), predominately from the glial cells (56\% in ages $0-14$ years, and $36 \%$ in all ages) [5]. Tumors of glial origin include glioblastomas, astrocytomas, oligodednrogliomas and ependymomas. Metastatic tumors are those that begin as cancer in one place and then spread to another. The most common types of metastatic cancers that spread to the brain are breast cancer and lung cancer. Currently, in children with primary CNS tumors diagnosed between the ages of 0 
and 14 there is a 5 year survival rate of $66 \%$. Of these $66 \%$, it has been reported that up to $86 \%$ will survive out to 15 years post-diagnosis [6]. With these advances in our ability to effectively treat tumors, it is becoming more and more important to identify the necessary procedures to limit the side effects of radiation treatment.

In the clinic, there are a variety of ways that radiation is used for treatment. The most common type of radiation treatment is external beam irradiation, where the radiation source is outside the body and must penetrate the body to reach its target. Other types of radiation therapy include: internal radiation or brachytherapy where a radioactive pellet is implanted at a specific site to treat a local area; systemic radiation where a radioactive source is injected into the veins and travels throughout the body delivering radiation; and interoperative radiation where the target is surgically exposed and then administered radiation using an external beam. For brain tumors, external beam radiation is used. However for the radiation to reach the tumor; it must pass through surrounding normal (or non-tumor) tissue, Figure 2.1. When the radiation passes through the normal tissue it damages the tissue, which can lead to side effects. The aim of the current work is to identify a way to minimize the damage to normal tissues while not compromising the effectiveness of radiation as a treatment for brain tumors.

\subsection{Side effects of radiotherapy and current treatments}

Radiation-induced CNS injury is usually divided into three categories: acute (within days), early (1-6 months post treatment), and late [7]. Late radiation damage has been identified through changes in neuropsychometric, neuroendocrine function, or vascular events, Figure 2.2, that may result in ischemia[8]. Brain tumor patients treated with cranial radiation have been reported to suffer from late-term side effects such as decreases in motor/sensory coordination, altered hypothalamic hormone levels, delayed growth and development, IQ deficits, memory loss, behavioral problems, and lower academic achievement [4], Table 2.1. These late-term effects are common in tissues with limited potential for self-renewal, such as in the CNS [3]. The observation of late radiation damage is often made in adults and children months or years after radiotherapy; however, we now know that the onset of microvascular changes after radiotherapy is immediate $[9,10]$. This chronological division of radiation injury does not necessarily reflect a mechanistic disparity $[11,12,13]$. A central hypothesis to our work is to understand the mechanisms involved in early radiation damage to better understand the development of the late damage. In this work, the role of the inflammatory response in the acute radiation damage is characterized.

During and after radiation, brain tumor patients are often treated with an array of medicines in an effort to relieve the acute side effects of radiotherapy, (Table 2.1), including steroids, diuretics to reduce brain swelling and pressure, anti-convulsants to reduce seizures, and pain medication. The main such treatment are corticosteroids such as dexamethasone. Dexamethasone is a glucocorticoid steroidal hormone that has antiinflammatory and immunosuppressant effects. It is administered to reduce edema and brain swelling; however it can produce side effects such as fungal infections, muscle weakness, fluid retention, and weight gain via increased appetite. All of the drugs administered are used to treat the physical side effects of radiation, and make the patient more comfortable. They are not intended to protect the irradiated tissue from damage, although 


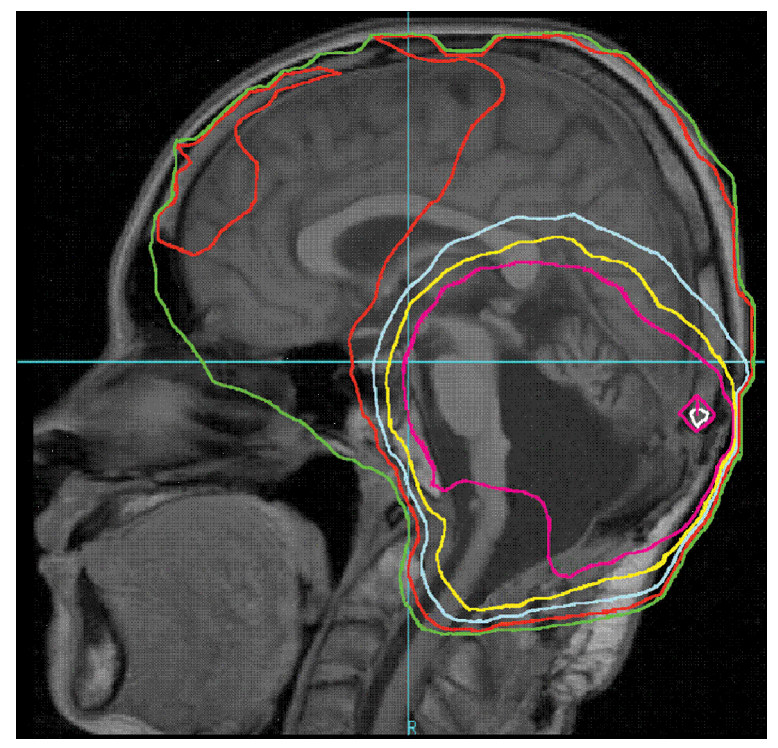

Figure 2.1: Radiation dose map.

Treatment plans are designed with the goal of maximizing radiation to the tumor volume, avoiding sensitive structures, and minimizing the dose to the non-tumor tissue. This image shows the treatment plan of composite whole brain and posterior fossa irradiation for a medulloblastoma. The colored boundaries are progressively lower doses of radiotherapy, with magenta $=55.8 \mathrm{~Gy}$; light blue $=36.0 \mathrm{~Gy}$; and green = 36.0 Gy. Image from Mulhern et al. "Late neurocognitive sequelae in survivors of brain tumours in childhood", Lancet Oncol, 5: 7, 2004, Copyright (c) 2004 Elsevier, with permission from the publisher.
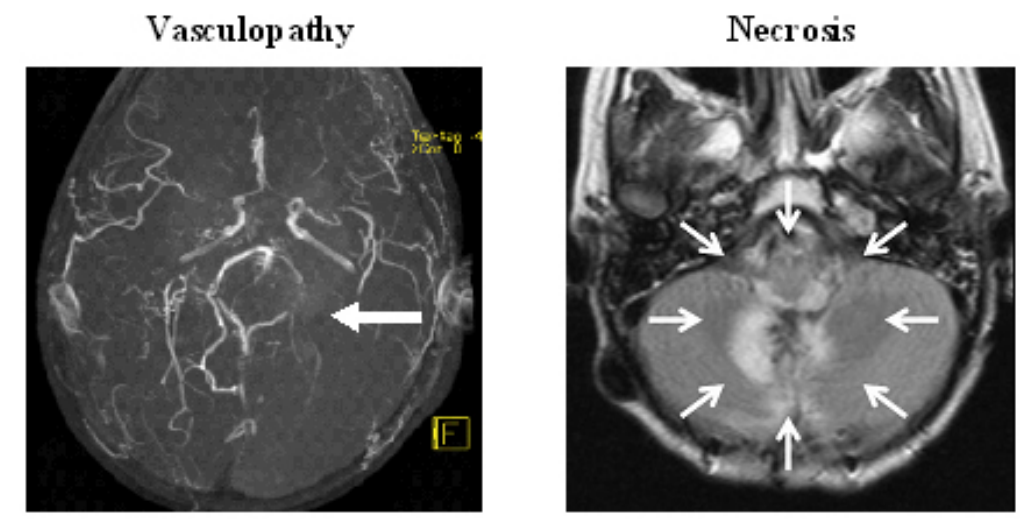

Figure 2.2: Late effects of radiation.

Using MR imaging, clinical late term effects can be observed. (Left) example of left posterior cerebral radiation-induced vasculopathy in a patient with low-grade glioma after radiation therapy and (Right) an example of brainstem and cerebellar radiation-induced necrosis in a patient with ependymoma (white arrows). Image courtesy of Dr TE Merchant, St Jude Children's Research Hospital 
Table 2.1: Clinical side effects of radiotherapy.

\begin{tabular}{cc}
\hline Acute & Long-term \\
\hline Nausea/vomiting & Neurologic: \\
Edema & Hearing loss \\
Fatigue & Loss of vision \\
Immune suppression & Motor/sensory coordination \\
Loss of appetite & Endocrine: \\
Hair loss & Growth and development \\
Dermatitis & Hypothalamic hormone levels \\
& Cognitive: \\
& IQ deficits \\
& Memory loss \\
& Attention disorders \\
& Behavior \\
& Academic achievement \\
\hline
\end{tabular}

treatment with dexamethasone has been shown to reduce permeability of the blood-brain barrier (BBB) $[14,15]$ and inhibit radiation-induced up-regulation of ICAM-1, P-selectin, and E-selectin [16].

\subsection{How radiation damages cells}

X-rays are the primary type of ionizing radiation used in the clinic. The x-rays are produced extranuclearly by accelerating electrons to a high energy and then stopping them abruptly in a target, most commonly of tungsten or gold [17]. For radiotherapy, linear accelerators are commonly used to produce the x-rays at megavoltage energies. The energy range used in clinical therapy is usually from 6-MV to 20-MV, with the higher energies used to obtain deeper penetration into the tissue.

The $x$-rays used in radiotherapy have enough energy to ionize atoms along their path through the tissue. As the x-rays traverse through the body, they interact with atoms along their path causing the ejection of electrons through one of three processes: photoelectric effect, Compton scattering or pair production. When the electrons are ejected, they form free radicals, primarily hydroxyl radicals, which can then damage the cells by causing single strand or double strand breaks in the DNA $[18,19]$. Single strand breaks can often be repaired when DNA is synthesized during cell replication. Double strand breaks, however, cannot be easily repaired and often result in cell death.

Following radiation, there are two types of damage that can occur, direct and indirect, Figure 2.3. At the cellular level, direct radiation damage is when the DNA of a cell is damaged directly by the penetrating radiation beams, most likely from the ionization of an oxygen molecule. Indirect damage is when a different molecule, usually water, is ionized creating free radicals that are able to damage the DNA of a cell [17]. At the system level, 


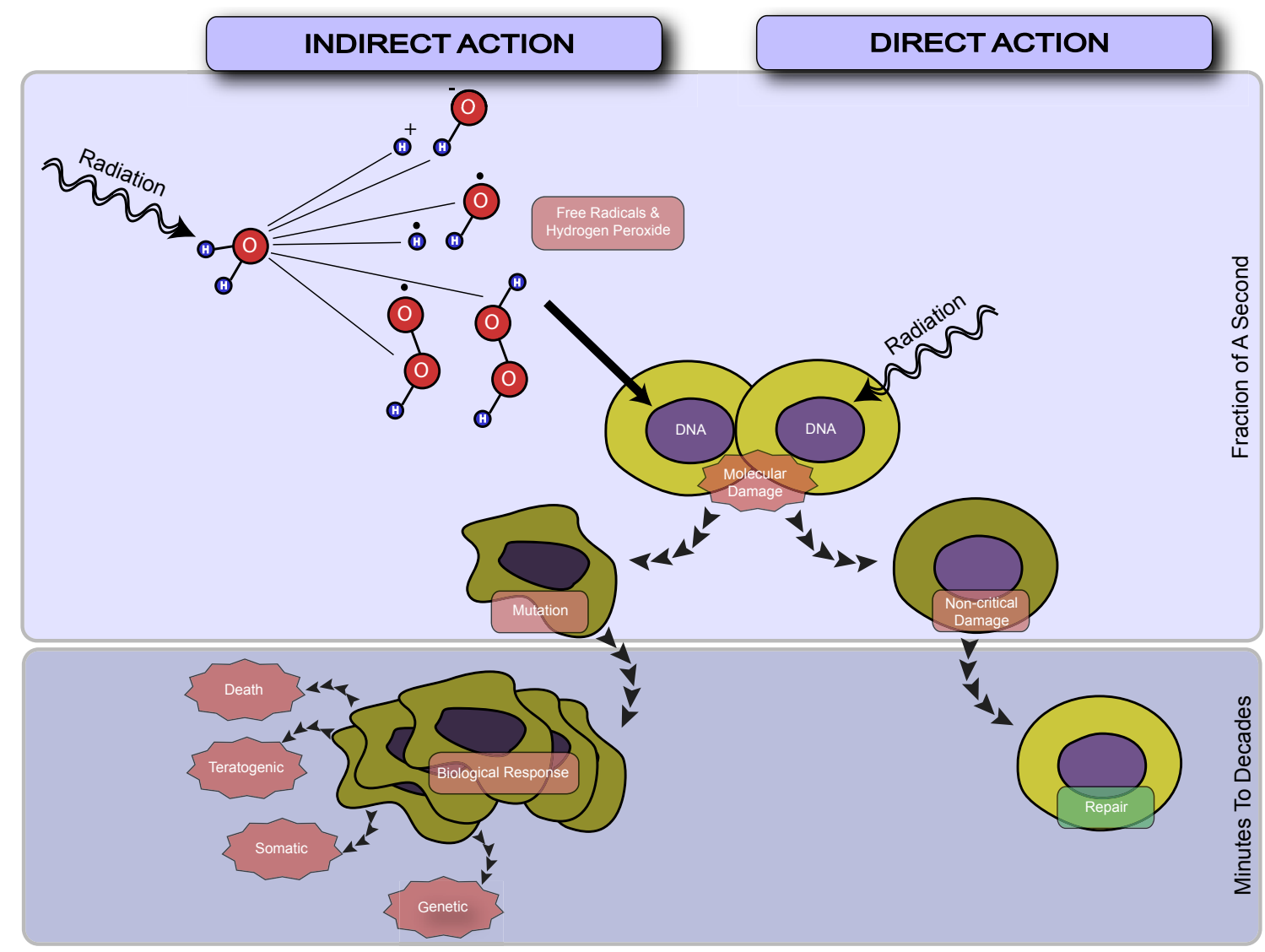

Figure 2.3: Direct versus indirect radiation.

Schematic of how radiation works. radiation enters the body, and primarily interacts with water molecules forming free radicals that then interacts with cells causing DNA damage, however there can also be direct damage to the DNA by the radiation. Once the DNA is damaged, the cell can either repair itself, or it will mutate and induce a biological response. This biological response can be death, or a genetic, somatic, or teratogenic effect. Note the time frame over which the damage can occur. 
direct damage is inflicted on a given cell by the ionizing radiation, while indirect damage is when a nearby cell is damaged initiating a sequlea of events that includes an inflammatory response and subsequently leads to damage of surrounding cells. Direct damage from ionizing radiation, is very difficult to prevent, but steps can be taken to minimize the indirect damage.

Organs are comprised of both vasculature and tissue; however, in the field of radiation research there is an ongoing controversy as to which, vasculature or issue, is the more significant source of radiation damage. Otsuka et al [20] used neutron radiation of mercapto-undecahydro-dodecaborane $(\mathrm{BSH})$ to selectively irradiate the vasculature of the brain, and then looked at neuronal precursor cell populations. They showed that when only the vasculature was irradiated there was less neuronal precursor cell loss indicating that neuron precursor cells are damaged more by direct radiation damage, than by indirect damage from the vasculature. Schuller et al [21] also showed that following selective radiation, administered similarly to Otsuka et al [20], there was no change in crypt stem cell loss compared to whole body radiation, indicating that it is direct not indirect damage. In contrast, Lyubimova et al [22] used the radioprotector Gammaphos, that does not cross the BBB, to show that when the vasculature is protected during radiation, the amount of necrosis, and endothelial cell loss, was significantly reduced. This indicates that the vasculature plays a primary role in radiation damage in the brain. Others have also shown that damage to the vascular endothelium is the primary event leading to late development of white matter necrosis [23]. In addition, there is also evidence that the extent to which vascular damage plays a role may depend on the location or organ being irradiated [24]. So although there is an ongoing controversy as to what role radiation-induced damage to the microvasculature network has on observed effects overall, there is agreement that it does play a part and as such, should be investigated.

\subsection{Sensitivity to radiation damage}

The sensitivity of an organ to radiation damage depends on: the inherit sensitivity of the individual cells, the kinetics of the tissue as a whole, and the way the cells are organized. In general, the tolerance of an organ to radiation depends on the ability of the clonogenic cells to maintain a sufficient number of mature cells suitably structured to maintain the function of the organ. As such, the total dose of radiation that can be tolerated by an organ depends on the volume of the organ receiving radiation. The amount of time between radiation insult and the expression of damage in the tissue/organ depends on the population kinetics of the organ. The population kinetics are based on the normal lifespan of the mature cells in the organ, and the amount of time it takes for new cells to mature to a functional state [17].

The radiosensitivity of an individual cell is related to the metabolic state of the cell; cells that have a higher rate of proliferation, such as tumor cells, are more radiosensitive than less proliferative cells such as neuronal or endothelial cells. It is this characteristic that makes radiotherapy a successful treatment for tumors. However, normal tissue is also damaged by radiation, but at a lower rate than tumor cells. 
Table 2.2: Classification of cell and tissue radiosensitivity.

\begin{tabular}{|c|c|c|}
\hline Radiosensitivity & Examples & Characteristics \\
\hline $\begin{array}{l}\text { High } \\
\text { radiosensitivity }\end{array}$ & $\begin{array}{l}\text { Cell: crypt cells, basal cells, } \\
\text { Type A spermatogonia } \\
\text { erythroblasts, lymphocytes } \\
\text { Tissue: lymphoid, marrow, } \\
\text { spermatogenic and ovarian } \\
\text { epithelium }\end{array}$ & $\begin{array}{l}\text { Rapidly dividing, } \\
\text { undifferentiated; does } \\
\text { not differentiate } \\
\text { between divisions }\end{array}$ \\
\hline $\begin{array}{c}\text { Relatively } \\
\text { radiosensitive }\end{array}$ & $\begin{array}{c}\text { Cell: myelocytes, } \\
\text { intermediate spermatogonia } \\
\text { Tissue: epidermal } \\
\text { epithelium, bladder } \\
\text { epithelium, optic lens, gastric } \\
\text { gland, and ureteral epithelium }\end{array}$ & $\begin{array}{c}\text { Actively dividing; } \\
\text { some differentiation; } \\
\text { differentiates between } \\
\text { divisions }\end{array}$ \\
\hline $\begin{array}{l}\text { Intermediate } \\
\text { radiosensitivity }\end{array}$ & $\begin{array}{l}\text { Cell: Endothelial cells, } \\
\text { fibroblasts cells Tissue: } \\
\text { interstitial connective, } \\
\text { neuroglial connective, fine } \\
\text { vasculature, bone tissue, } \\
\text { cartilage }\end{array}$ & $\begin{array}{l}\text { Irregularly dividing, } \\
\text { moderate mitotic } \\
\text { frequency }\end{array}$ \\
\hline $\begin{array}{l}\text { Relatively } \\
\text { radioresistant }\end{array}$ & $\begin{array}{l}\text { Cell: Parenchymal cells of } \\
\text { the liver, fine vasculature } \\
\text { Tissue: Mature cartilage or } \\
\text { bones, salivary glands, } \\
\text { respiratory organs, kidneys, } \\
\text { liver, pancreas, thyroid, } \\
\text { adrenal and pituitary glands }\end{array}$ & $\begin{array}{l}\text { Do not normally } \\
\text { divide, but retain } \\
\text { capability of division; } \\
\text { differentiated }\end{array}$ \\
\hline $\begin{array}{l}\text { Highly } \\
\text { radioresistant }\end{array}$ & $\begin{array}{c}\text { Cell: Nerve cells, muscle } \\
\text { cells, Erythrocytes (RBC's) } \\
\text { Tissue: Muscle, Brain, Spinal } \\
\text { Cord }\end{array}$ & $\begin{array}{l}\text { Do not divide; } \\
\text { differentiated }\end{array}$ \\
\hline
\end{tabular}


lonizing radiation can damage any type of cell in the body, however, the sensitivity of a cell to damage by radiation depends on multiple factors including the type of cell and the phase of the cell cycle that particular cell is in. Table 2.2, shows the basic relative radioresistance of different cells based on direct radiation effects. Endothelial cells, the cells that comprise the vasculature of the brain, are considered intermediate in radiosensitivity, while neuronal cells are some of the most radioresistant cells in the body. In addition to cell type, the phase of the cell cycle that a cell is in affects its radiosensitivity. Cells are the most sensitive during mitosis (M phase) and RNA synthesis (G2), less sensitive during the pre-DNA synthesis period (G1), and least sensitive during DNA synthesis (S Phase) [18]. If a cell is physically damaged by radiation, it can repair itself, mutate, or die which leads to the biological expression (or effects) of the damage. This biological expression may appear within seconds to minutes of damage, or it may take months to years for an effect to be observed.

The conventional approach to radiotherapy is to administer multiple small doses of radiation to a region over a period of weeks as a way to limit damage to normal tissues and to increase damage to the tumor. This works for four reasons: 1) it allows time for the repair of sublethal damage to normal cells, 2) it allows for the re-population of cells, 3 ) it allows a re-assortment of cells within phases of the cell cycle, and 4) it allows for the reoxygenation of the region [17]. By allowing the normal (non-tumor) cells time to repair after insult, there is an increased chance that the cell will survive, and if the interval is long enough, repopulation of a cell type can occur. As the sensitivity of a tissue depends on the organ maintaining enough mature functioning cells, allowing time for the injured cells to repair themselves increases the tolerance of an organ to radiation. In addition, as radiation relies on oxygen to create the free radicals that damage a cells' DNA, allowing time for reoxygenation means that when a subsequent dose of radiation is given, you are not reducing the overall effectiveness of the radiation to kill the tumor cells. Also, as tumor cells are highly proliferative, allowing time between radiation doses increases the number of cells that will be in the most sensitive phases (M and $\mathrm{G} 2$ ) of the cell cycle.

\subsection{The brain: components and response to radiotherapy}

The brain plays an important role in coordinating motion, interpreting, and storing sensual information. Such complicated functions demand a continuous and reliable supply of oxygen and nutrients (e.g. glucose). The overall design of the microvascular network in the brain is a function of metabolic stimuli, biochemical growth factors, feedback control, external stimuli, and hemodynamic parameters [25]. The network has a versatile architecture that responds dynamically to the functional demands of the tissue, and is used by the immune system to monitor and survey its surrounding tissue and as the highway through which relief and repair is delivered. The microvascular network is defined as a series of interconnected arterioles, venules, and capillaries with low Reynolds numbers and large surface areas, which provide an optimal environment for the supply of oxygen, nutrients, removal of waste products and interaction of blood cells (i.e., red blood cells, white blood cells, platelets, etc.) with endothelial cells [26, 27]. The diameters of the blood vessels range in size with the arteries being $10-125 \mu \mathrm{m}$, venules $20-100 \mu \mathrm{m}$, and capillaries 7 $20 \mu \mathrm{m}$, with the blood velocity varying between $100-2000 \mu \mathrm{m} / \mathrm{s}$ within these vessels [28]. 
This process is more complex in the brain because of the presence of the blood-brain barrier (BBB), Figure 2.4. The BBB is responsible for protecting the parenchyma of the brain at the vascular interface, by regulating the movement of substances between vessels and tissue. The BBB is comprised of endothelial cells that are tightly adhered via tight junction proteins such as occludin. In the brain's vasculature, these tight junctions eliminate the paracellular pathway for hydrophilic solutes, which is otherwise available in vasculature outside of the brain [29,30]. The endothelial cells that comprise the BBB also have very few pinocytotic vesicles [31] and fenestrations that are common in non-cerebral vasculature, and contain more mitochondria [32] than normal endothelial cells, indicating more specific active transport of nutrients from the blood, rather than passive transport. The BBB is regulated by the presence of astrocytes and pericytes [33, 34, 35]. Pericytes are phagocytic cells that remove foreign bodies, while astrocytes are star-shaped glial cells whose end-feet surround the endothelial cells strengthening the tight junctions and providing another layer of protection for the parenchyma from substances in the blood. When the barrier between the vascular supply of the brain and the CNS parenchyma is disrupted, excess extravasation of proteins, biologic-response molecules (e.g. growth factors, cytokines, and clotting factors), inflammatory cells, and therapeutic drugs can damage the brain [36, 37, 38, 39]. The disruption of the BBB has been identified as a consequence of various diseases/injuries such as multiple sclerosis, ischemia, HIV, hypertension, brain tumors, CNS injury and radiation exposure [39, 40, 41, 42, 43, 44, 45, 46]. Following radiation, the BBB is known to be damaged. There is opening of the tight junctions leading to increased vessel permeability, up-regulation of adhesion molecules leading to increased leukocyte activity, astrocytes and pericytes pull away from the endothelial cells, Figure 2.4, Table 2.3. The reported radiation-induced late effects on the microvasculature include telangiectasias, dilation, vessel wall hyalinization, and thickening with fibrinoid necrosis $[47,48]$. Damage to any part of the BBB may compromise its integrity and ability to protect the parenchyma.

We and others have demonstrated that there is an increase in BBB permeability following cranial radiation $[9,70,71,72]$. This increase in permeability is due, at least in part, to changes in the integrity of the tight junctions between adjacent endothelial cells and by the formation of vesicles in the endothelial cells. Yuan et al have shown that there is a size dependent increase in BBB permeability 24-hrs after cranial radiation [9]. Yuan et al also showed that there is a time-dependent difference in the mechanism of BBB permeability. In the acute phase following radiation, alterations of the tight junctions have been observed as the mechanism responsible for the BBB permeability increase, while in the late phase, the primary mechanism appears to be vesicular formations [70]. The alterations of the tight junctions are thought to be caused by the up-regulation of intracellular adhesion molecule1 (ICAM-1), which is known to induce cytoskeletal reorganization. The effect that radiation has on endothelial cells themselves and the role they play in initiating/sustaining damage is of great interest. It has been shown that when irradiated, endothelial cells undergo apoptosis at a higher rate than other cells, in vitro and in vivo [50, 51, 73].

Endothelial cells play a unique role in maintaining the body's physiological balance or homeostasis, controlling the movement of substances across the blood compartment into the different tissues and organs with varying demands and function [74]. The endothelial cells also play an important immune function through leukocyte surveillance and extravasation by regulating adhesion integrins and cytokine production [75]. We and others have shown that there is an increase in leukocyte activity following radiation $[76,77,78$, 


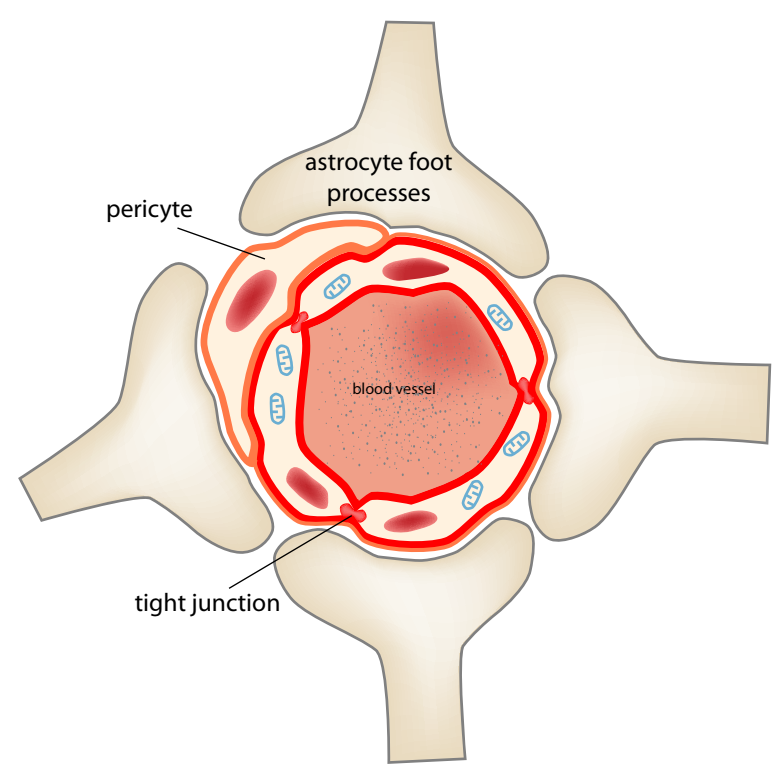

Normal Blood-Brain Barrier

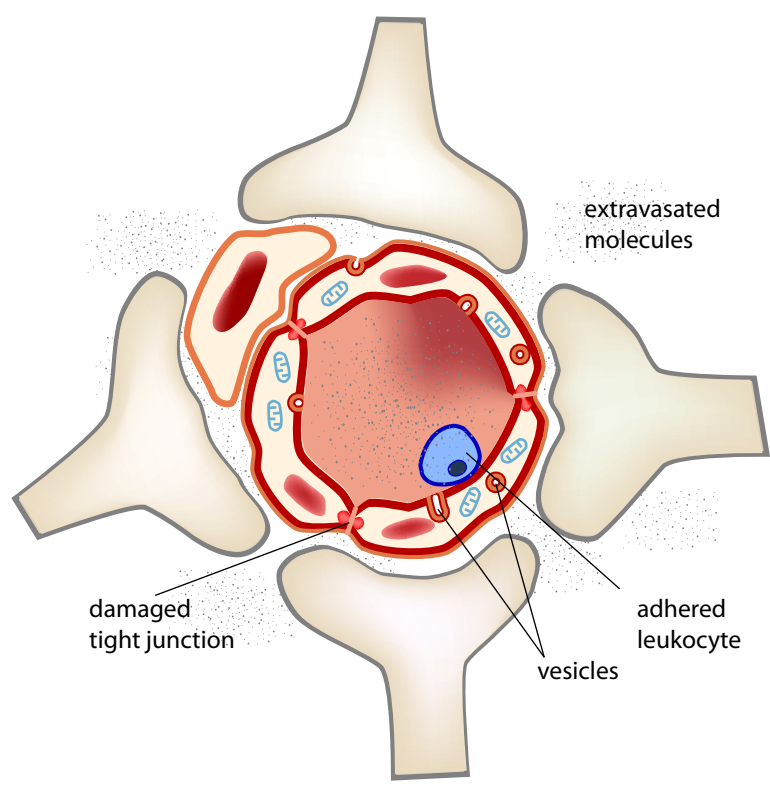

Damaged Blood-Brain Barrier

Figure 2.4: Cartoon depiction of the normal and damaged BBB.

A cartoon depicting a cross section of brain parenchyma showing the structure of the blood brain barrier (BBB) and the damage induced by radiotherapy. (Upper) Normal BBB showing: intact tight junctions; lack of vesicles; astrocytes and pericytes abutting the endothelial cell providing additional barrier support. (Lower) Damaged BBB; astrocytes and pericytes have pulled away from the endothelial cell; a leukocyte has adhered to the endothelial cell surface; loss of tight junction integrity; and vesicle formation. The damage to the BBB results in the extravasation of molecules out of the blood vessel and into the extracellular space. 
Table 2.3: Radiobiologic side effects of radiotherapy.

\begin{tabular}{cc}
\hline Reported Side Effect & Reference \\
\hline Increased vessel permeability & {$[49,50,51]$} \\
Altered expression of adhesion molecules & {$[52,16]$} \\
Vascular constriction & {$[10]$} \\
Platelet aggregation & {$[53]$} \\
Endothelial detachment from the basement membrane & {$[53]$} \\
Cytoskeletal reorganization & {$[54]$} \\
Decreased vessel density & {$[55]$} \\
Altered cytokine expression & {$[56,57,58]$} \\
Inhibition if angiogenesis & {$[59]$} \\
Hypoxia & {$[60,61]$} \\
Loss of neuronal precursor cells & {$[23,62,63]$} \\
Decrease in cell proliferation & {$[64]$} \\
Apoptosis & {$[64,51]$} \\
Thromboses & {$[65]$} \\
Demyelination & {$[66,7]$} \\
Necrosis & {$[66,67,68]$} \\
Astrogliosis & {$[69,58,70]$} \\
\hline
\end{tabular}


30, 9, 26]. When initially inflamed, endothelial cells will up-regulate numerous adhesion molecule receptors, L-selectin, E-selectin, and P-selectin, on their surface. The function of these receptors is to weakly attract leukocytes, which causes them to roll along the endothelium initiating the first step in leukocyte-endothelial interactions. The second step in leukocyte-endothelium interactions is the adherence of the leukocytes to the endothelium, a rate-controlling step in the inflammatory cascade, which is mediated by the upregulation of adhesion molecules (ICAM-1), intracellular adhesion molecule-2 (ICAM-2), vascular cell adhesion molecule-1 (VCAM-1), and platelet/endothelial cell adhesion molecule-1 (PECAM-1) on the endothelium [79]. Yuan et al have shown that following cranial radiation, there is an increase in the expression of ICAM-1, E-selectin, and P-selectin, and that the expression of these molecules is time-dependent. It is known that certain cytokines are responsible for an increase in leukocyte adhesion such as: tumor necrosis factor-alpha (TNF), Interleukin-1, Interleukin-4, and Interferon- $\gamma[80,79]$.

A decrease in arteriole diameter is one of the reported manifestations of radiationinduced damage to the microvascular network [10]. The mechanisms of vasoconstriction are not completely understood, but include: alterations in calcium signaling from the astrocytes, changes in nitric oxide levels, constriction of the smooth muscle cells, hematocrit variations, and over-proliferation of endothelial cells [81, 17, 82, 83, 84]. When vasoconstriction occurs, the amount of oxygen reaching an area is reduced creating a state of hypoxia and inducing the up-regulation of hypoxia inducible factor- $1 \alpha$ (HIF-1 $\alpha$ ). Hypoxia has been shown to activate inflammatory mediators $[85,86]$, including ICAM-1, on endothelial cells, and promote vascular remodeling/angiogenesis [85] and vascular permeability $[60,87]$ via the up-regulation of vascular endothelial growth factor (VEGF) and its receptors. Hypoxia also creates a shortage of oxygen free radicals, which is primarily how ionizing radiation damages DNA, which can increase the radioresistance of nearby cells $[61,88,89,90]$.

Astrocytes are the most abundant cells in the CNS, outnumbering neurons 10 to 1 $[91,92]$. They have many roles in the brain including helping to maintain the integrity of the $\mathrm{BBB}$, supplying nutrients to neurons, regulating ion concentrations in the extracellular space, signal transmission and controlling vascular hemodynamics [93]. In response to CNS injury, astrocytes will become activated and respond by hypertrophy of their cellular processes and an increased production of the intermediate filament glial fibrillary acidic protein (GFAP) [94, 95]. Astrocyte activation, or astrogliosis, can persist from weeks to months to years after an injury. Previous studies have shown that astrogliosis occurs following a single dose [69] or fractionated doses of radiation [70] and that astrocytes can produce the inflammatory markers tumor necrosis factor-alpha (TNF) and ICAM-1 which are also known to be up-regulated following radiation $[57,96]$. Whether radiation-induced astrogliosis is caused by direct ionization damage or due to the breach in the BBB exposing the parenchyma to noxious substances and inflammatory cytokines is unclear. However, activated astrocytes have been shown to secrete inflammatory agents [97, 98] which, in turn, could amplify endothelial cell and microvascular damage.

The neurons are less abundant than glial cells in the parenchyma and are responsible for transferring signals across the brain's functional systems [99, 38]. Neuronal cells are responsible for processing and transmitting cellular signals through chemical and electrical synapses. In the brain, neuronal axons are usually covered with an insulating layer formed by glial cells, known as oligodendrocytes, to form the so called myelin sheath. 
This sheath increases rates of signal transmission and confines the signal to the neuron thus conserving metabolic energy. The demyelination of neurons can be dire and is seen in neurodegenerative autoimmune diseases such as Multiple sclerosis, Transverse myelitis, Guillain-Barre Syndrome, and adrenoleukodystrophy. In these diseases the inflammatory cells are able to penetrate the BBB and destroy the myelin surrounding the axons. Demyelination and myelin thinning have been reported in the CNS following radiation [100, 101, 102, 103]. In addition, Felts et al. has also shown prolonged increases in BBB permeability can induce demyelination of neurons [104].

Mature oligodendrocytes, the glial cells that lay the myelin sheath insulating the neuronal axons in the brain, are derived from the oligodendrocyte type 2 astrocyte $(\mathrm{O}-2 \mathrm{~A})$ progenitor cells. A decrease in O-2A cell populations limits the capacity to form new oligodendrocytes. After radiation, it has been shown that there is a decrease in the number of clonogenic $\mathrm{O}-2 \mathrm{~A}$ cells as well as oligodendrocytes. Oligodendrocytes have been shown to undergo apoptosis via the p53 pathway, while the mechanism of cell loss of O-2A cells is unknown. It has also been shown that oligodendrocyte cell death can be induced by TNF, which is up-regulated following radiation, thus adding an additional toxicity to the cells following radiation exposure. The loss of the O-2A cells following radiation has been proposed as a cause for the observed demyelination [105].

As is expected, long-term damage from radiotherapy involves the many different cell types found in the brain as well as circulating cells. Figure 2.5 is a graphical depiction of the interaction of the components of the brain and their role in observed damage following cranial irradiation. The current work focuses on the acute vascular and astrocytic responses to radiation damage, with the goal of understanding the mechanism of this damage so that an interventionl therapy can be used to minimize the response. It is hypothesized that by minimizing the acute vascular and astrocytic response the long-term response can be abrogated.

\subsection{Radiation-induced inflammation and current clinical treatments}

The up-regulation of adhesion molecules plays an important role in the inflammatory response of normal vasculature and facilitates the recruitment, transendothelial migration and proliferation of inflammatory cells in the extravascular compartment $[106,107$, 108, 109]. TNF is a soluble $17 \mathrm{kDa}$ pro-inflammatory cytokine molecule that binds to two distinct receptors, TNFR1 (p55) and TNFR2 (p75). It is primarily produced by activated macrophages and monocytes, however, in the brain; all brain cells including endothelial cells have the ability to produce TNF in response to stimulus $[110,111]$. TNFR1 is known to be ubiquitously expressed on all mammalian cells while TNFR2 is largely confined to endothelial cells. TNF is known to be involved in leukocyte adhesion, cell permeability, edema, acute and chronic inflammation, and the release of other inflammatory cytokines $[112,113]$. Binding of TNF to its receptors has been implicated in the activation of nuclear factor-kappa $\mathrm{B}(\mathrm{NF}-\kappa \mathrm{B})$, which in turn switches on the synthesis of adhesion glycoproteins such as ICAM-1 [80], Figure 2.6. In addition to activating NF- $\kappa \mathrm{B}$, TNF can induce cytoskeletal reorganization leading to the up-regulation of P-selectin from the Weibel-Palade bodies. When inactive, P-selectin is internalized, but when activated it moves to the cell surface where it is a primary molecule responsible for cell rolling, along with E-selectin. 


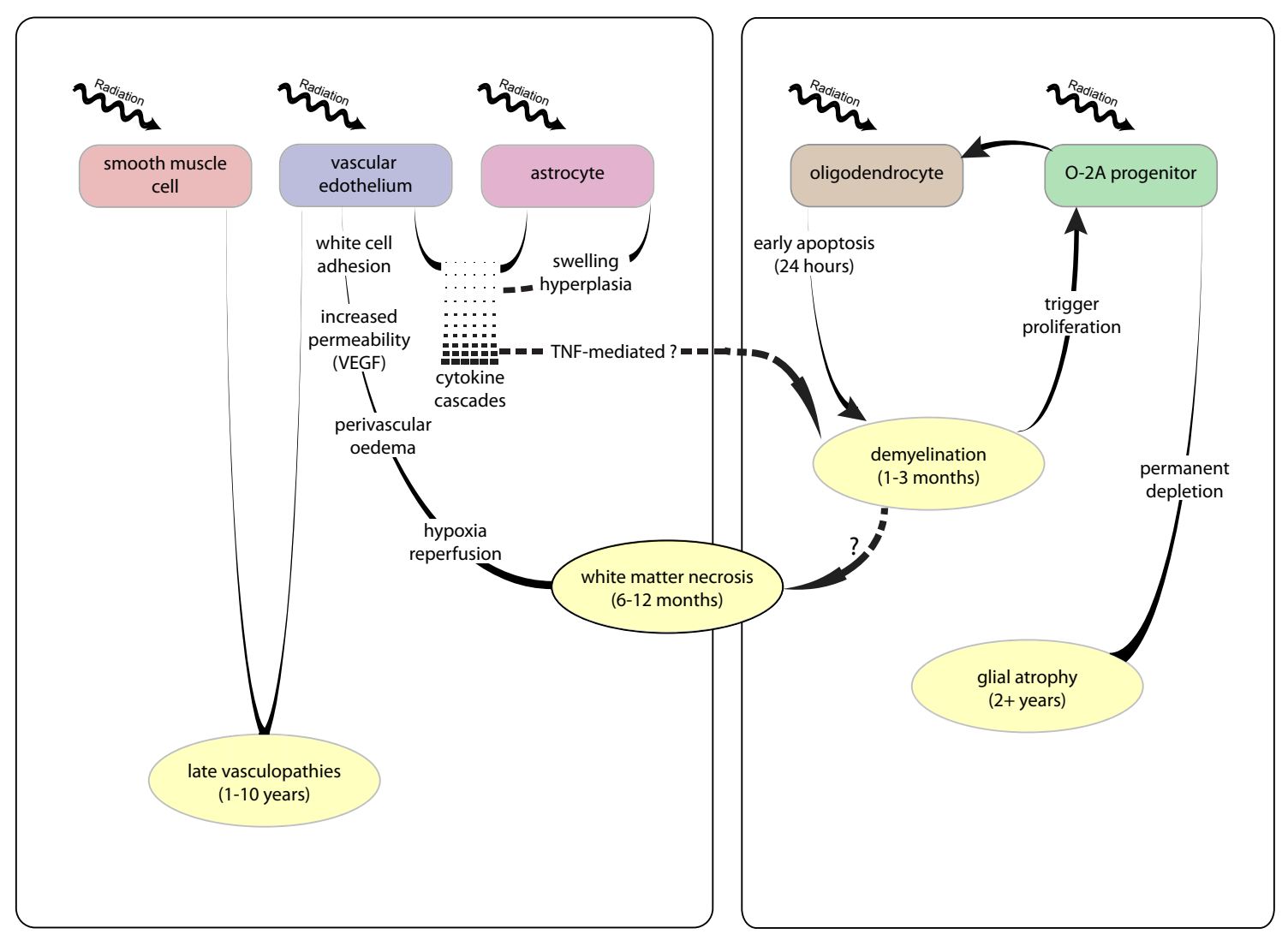

Figure 2.5: Pathophysiology of radiation-induced damage.

This is a schematic of the different cell types in the brain and their hypothesized roles in the induction of radiation-induced lesions in the brain. Adapted with permission. G. Steel, Basic Clinical Radiobiology 3rd edition, Oxford University Press, p. 37. Copyright (c) 2002. Arnold Publishers. 


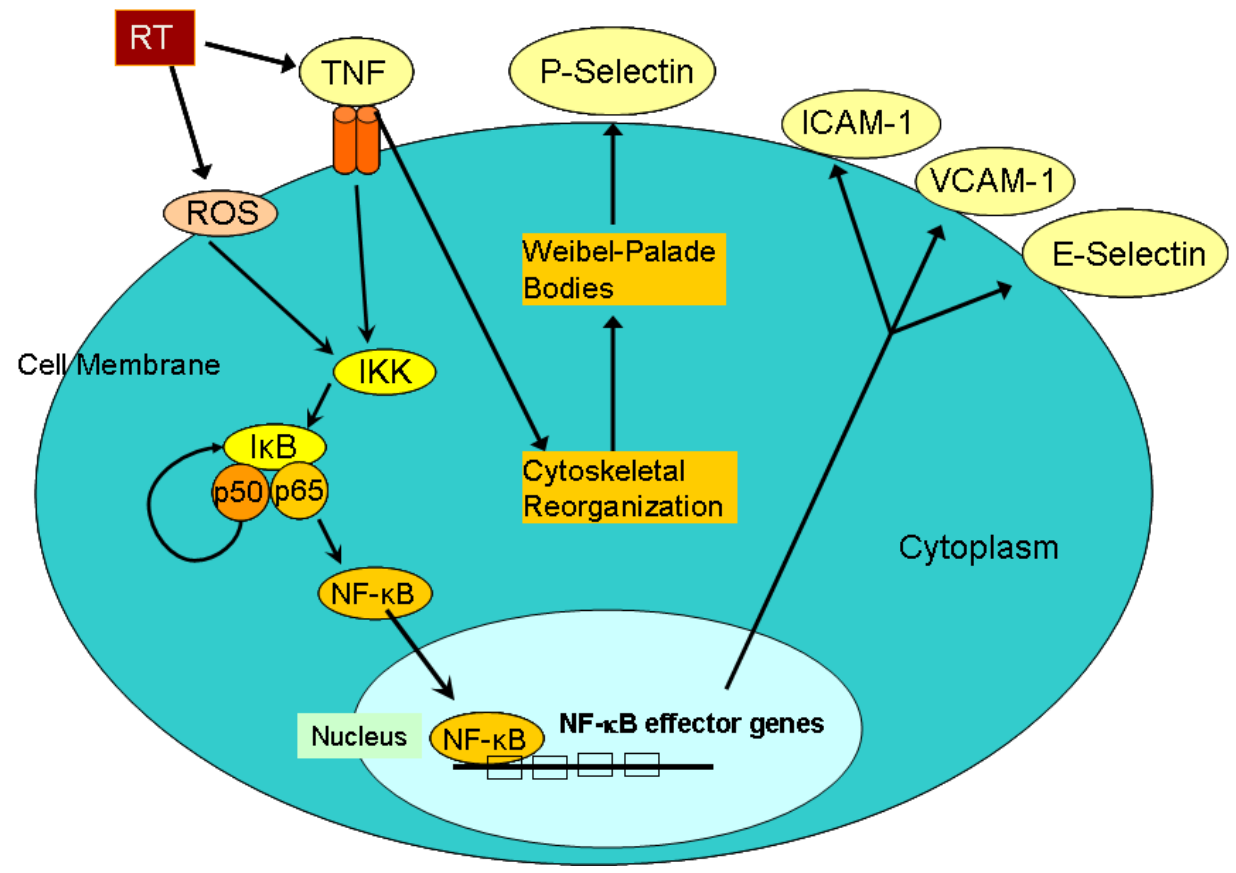

Figure 2.6: Radiation-induced cellular response.

Diagram of the main inflammatory molecules known to be induced by radiation, and how they interact. 
TNF has been shown to be up-regulated at the mRNA level following single and fractionated radiation [57].

ICAM-1 is a cell surface glycoprotein that is up-regulated by inflammatory stimuli and plays a role in a variety of diseases by interfering with normal immune function [109]. It is constitutively expressed in the brain by endothelial cells, astrocytes, and microglia [109]. ICAM-1 is the primary adhesion molecule responsible for the transendothelial migration of leukocytes from vessel to surrounding tissue by inducing cytoskeleton restructuring creating openings between adjacent endothelial cells [114]. These openings allow not only leukocytes, but any substance that is smaller than the opening created to enter the parenchyma from the blood. Previous studies have shown that radiation induces the up-regulation of ICAM-1 gene and protein expression in the brain [57].

$\mathrm{NF}-\kappa \mathrm{B}$ is a transcription factor that plays significant roles in cell proliferation, apoptosis, stress response, inflammation, and other cellular processes. Following radiation, the activation of TNF and the creation of reactive oxygen species (ROS), lead to the activation of the $\mathrm{I} \kappa \mathrm{B}$ kinase $(\mathrm{IKK})$ which then degrades the inhibitor of $\kappa \mathrm{B}(\mathrm{I} \kappa \mathrm{B})$. The degradation of $1 \kappa \mathrm{B}$ leads to the release of two NF- $\kappa \mathrm{B}$ subunits, p65 and $\mathrm{p} 50$. The p50 subunit acts as a regulatory control and re-inhibits NF- $\kappa$ B by promoting $l \kappa \mathrm{B}$, while the p65 subunit travels to the nucleus to activate the transcription of genes with binding sites for NF- $\kappa \mathrm{B}$ activity. Genes that are known to be regulated through NF- $\kappa$ B include ICAM-1, VCAM-1, and Eselectin $[115,116]$. Further, there is evidence that TNF and NF- $\kappa \mathrm{B}$ are major targets of commonly used anti-inflammatory drugs such as glucocorticoids and aspirin [80].

\section{$2.7 \quad K Z-41$}

$\mathrm{KZ}-41$ is a potent analog of quinic acid, which is an active ingredient in hot water extracts of the herbal cat's claw (uncaria tomentosa). Hot water cat's claw extracts (e.g., C-Med-100® and Activar $A C-11 \AA)$ have very low alkaloid content $(<0.5 \%)$ and yet retain significant anti-inflammatory activity, which can be attributed to the quinic acid carboxy alkyl ester [117]. Studies have shown that the anti-inflammatory activity of cat's claw extracts is through the inhibition of $\mathrm{NF}-\kappa \mathrm{B}$, and that this inhibition is coming from the quinic acid carboxyl alkyl ester found in the extract [118]. Aqueous extracts from the bark or root bark are used therapeutically in a diversity of diseases including allergy, arthritis, chemotherapy side effects, cancer, bacterial/fungal infections, gastrointestinal inflammation and gastric ulcers $[119,120,121,122,123]$. Oral administration of C-Med-100® to humans at doses of $250-750 \mathrm{mg}$ daily has been shown to enhance immune cell response and DNA repair $[124,125,126]$.

Preliminary studies, unrelated to the current work, indicate that $\mathrm{KZ}-41$ has potent vascular anti-inflammatory effects, promotes vascular recovery from injury, and increases white blood cell count, making it an excellent candidate as a potential agent that facilitate tissue recovery and wound repair. In a preliminary study using a dorsal window model, C57BL/6J mice received 7-Gy total body irradiation followed by a vascular wound on 5 days post-irradiation. FITC-dextran 150-kDa was used to evaluate blood flow and vessel perfusion, and Rhodamine-6G was used to evaluate leukocyte activity. The results, Figure 2.7, showed that injured vessels in unirradiated animals clotted immediately, had minimum 


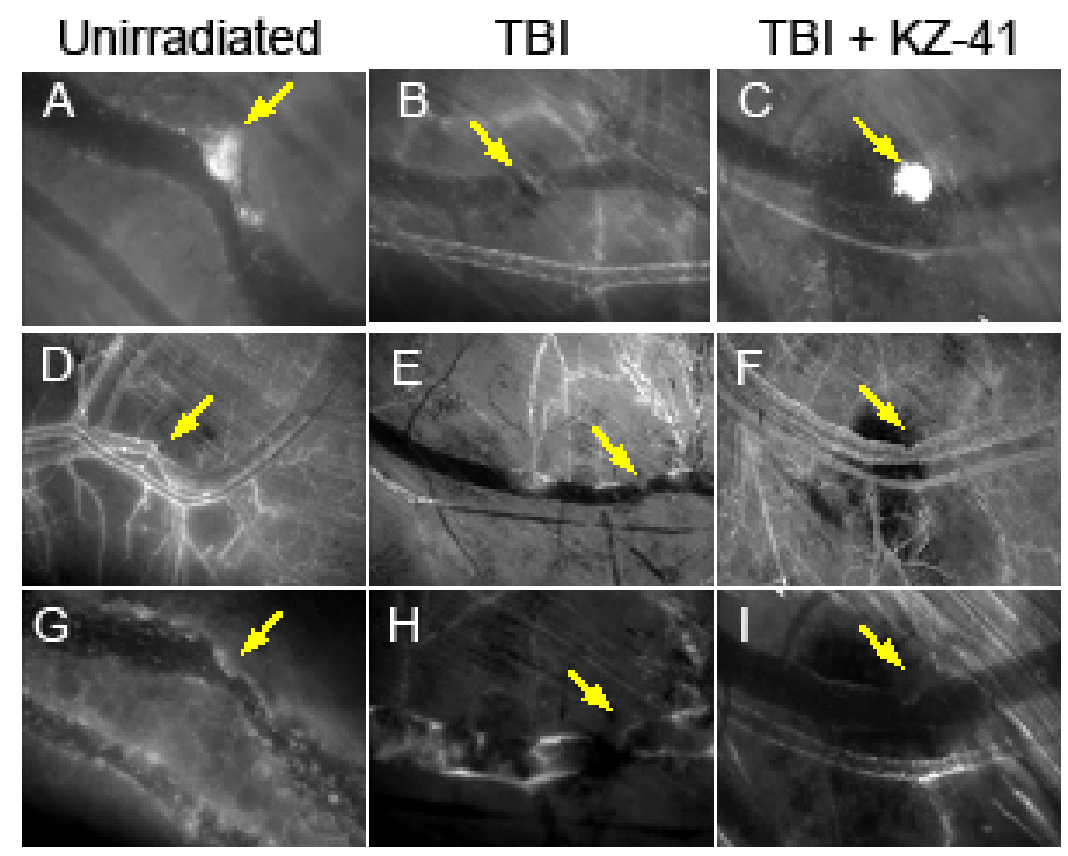

Figure 2.7: Effect of KZ-41 on vascular injury.

KZ-41 restores microvascular flow following radiation combined injury at the vascular level. The images shown are of a vascular wound (a nick in the vessel) in the dorsal window of animals that received 7-Gy TBI (total body irradiation). The vascular wound was made 5 days post-TBI and 3 days post-window placement. Top and bottom rows show vessels immediately and 24-hrs post-wound (200X) after injection of rhodamine-6G which stains leukocytes. Note the lack of clot in the irradiated animal's microvessel $(B)$ and the restoration of the clot in the treated animal (C). Center row: 24-hrs post-wound following injection of FITC-dextran 150-KDa (100X). 150-kDa FITC-dextran was used to evaluate vessel perfusion, as the molecule is large enough that there is minimal extravasation. Note that perfusion was restored within seconds in control animals (D), was diminished in irradiated animals $(E)$, and similar to control in irradiated animals treated with KZ-41 (F) (both $E$ and $F$ are acquired at 3 min post-dye injection). Leukocyte activity was lacking in both TBI (H) and $\mathrm{TBI}+\mathrm{KZ}-41$ treated $(\mathrm{I})$ as compared to control $(\mathrm{G})$ at 24-hrs post-wound. 
alteration in blood flow or vessel perfusion, and had a significant amount of leukocyte activity. The restoration of the vessel was seen by 24 -hrs after injury. In contrast, animals that received total body irradiation showed no clot formation at any time, blood flow was slowed and changing direction, minimal leukocyte activity, and by 24-hrs after injury, blood flow was being diverted to surrounding vessels. In animals treated with KZ-41 (intraperitoneal $(100 \mathrm{mg} / \mathrm{kg}$ ) daily beginning the day of irradiation) in addition to the total body irradiation, there was an immediate clot formation, and blood flow and vessel perfusion were comparable to the unirradiated animals by 24-hrs after injury, however leukocyte activity was low compared to the unirradiated group. The effects that were observed in vivo were confirmed using blood chemistry, Figure 2.8. with KZ-41 effectively limited leukopenia (loss of leukocytes) at 4 days following total body irradiation. It was also observed that total body irradiation induced a significant decrease in weight, and that treatment with KZ-41 prevented this loss, Figure 2.9.

The vascular system plays a clear role in wound healing through its delivery of oxygen, immune cells and metabolites to the injured site and through debridement of the injured site during the inflammation phase of healing to allow for proliferation to start. Direct damage to the vascular bed through mechanical injury, or indirect through radiation toxicity or through infection would interrupt these vital functions and delay wound repair. In this work, the antiinflammatory effects of KZ-41 are explored as a possible interventional therapy following cranial radiation.

\subsection{Gene expression}

Deoxyribonucleic acid is a nucleic acid that contains the genetic instructions used in the development and functioning of all known living organisms. DNA consists of a chain of nucleotide subunits of four types: adenine, cytosine, guanine, and thymine. Each subunit is made up of a phosphate group, a deoxyribose sugar ring, and a nucleobase. The way in which the subunits are linked in the chain gives the DNA molecule a polarity with a hydroxyl group on one end ( 3 ' end) and a phosphate group on the other end ( 5 ' end). A DNA molecule is composed of two polynucleotide chains held together by hydrogen bonds. The most common form for DNA is in a double helix structure where guanine pairs with cytosine via three hydrogen bonds and adenine pairs with thymine via two hydrogen bonds. The sugar-phosphates form the backbone of the double helix structure and each turn of the helix contains 10 base pairs.

The expression of genes encoded in the DNA begins by transcribing the gene into RNA (ribonucleic acid). RNA is similar in structure to DNA except that there is a ribose sugar in the subunit versus deoxyribose, there are uracils instead of thymines as a nucleobase, and RNA is single stranded not double stranded.

During transcription, the enzyme, RNA polymerase, copies a given sequence of nucleotides from DNA into a nucleotide sequence of RNA. Once the RNA sequence is made, a type of RNA called messenger RNA (mRNA) carries the information to the ribosome of the cell. Inside the ribosome, there is ribosomal RNA ( $r R N A)$ and transfer RNA (tRNA). The ribosomal RNA forms the core of the ribosomes, where mRNA is translated into pro- 


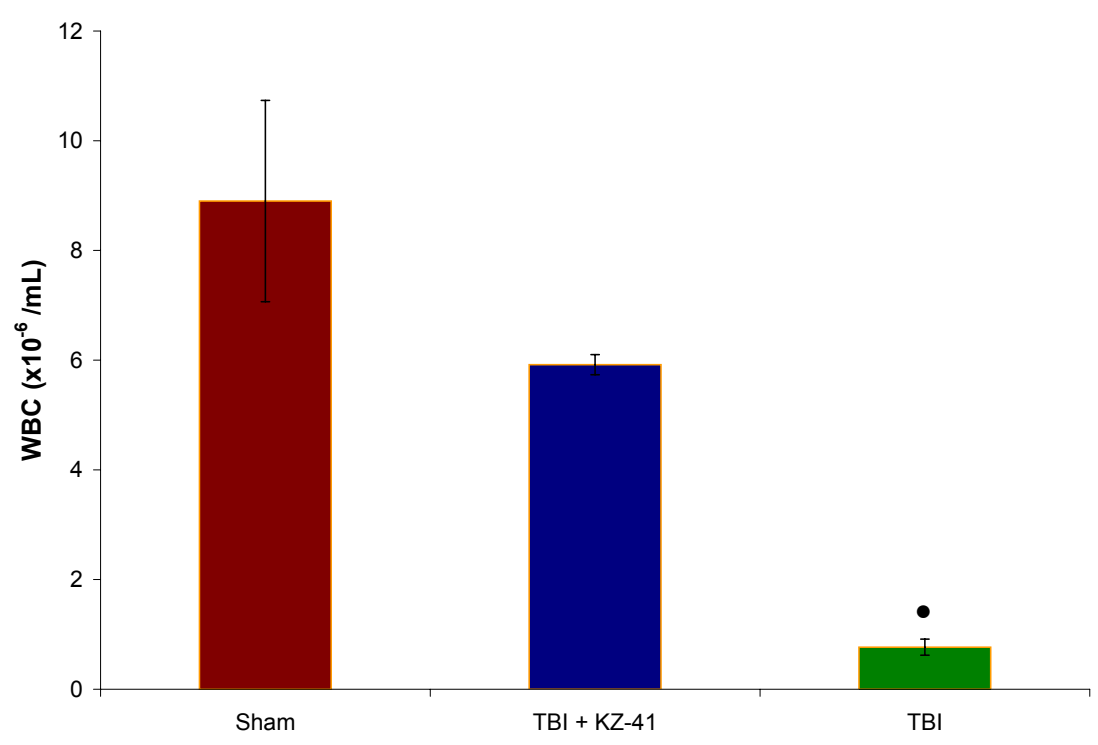

Figure 2.8: KZ-41 inhibits leukopenia.

Treatment with KZ-41 maintained total white blood cell counts at 4 days post-TBI. $(n \geq 3)$. - indicates a significant difference $(\mathrm{P}<0.01)$ compared to sham and TBI + KZ-41.

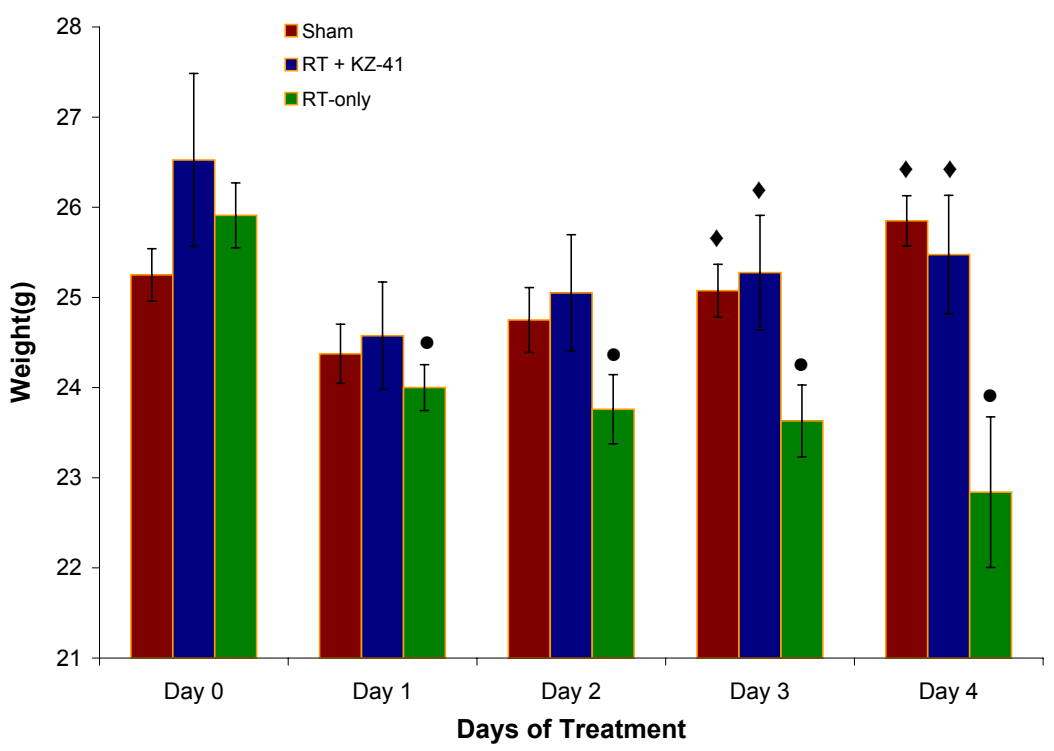

Figure 2.9: KZ-41 prevents weight loss following total body irradiation.

Graph of animal weights following TBI. All animals (except shams) received 7Gy TBI on Day 0. • indicates a significant difference $(P<0.01)$ within that group compared to Day 0. indicates a significant difference $(\mathrm{P}<0.05)$ compared to TBI-only at the specified time point. $(n \geq 4)$. 
tein, while transfer RNA forms adaptors that select amino acids and holds them in place so they can be incorporated into a protein.

A cell can change the expression of its genes in response to an external signal. This change can occur in multiple places between the DNA level and protein level. The most common type of control is transcriptional control, where the frequency and time at which a gene is transcribed can be controlled. Other locations for the control of gene expression are, RNA processing and transport control, where the messenger RNA is not allowed to carry the transcribed information to the ribosomes, translation control, where the mRNA is not allowed to be translated into protein, or by selectively activating or inactivating proteins after they have been made. In addition to controlling whether a gene is transcribed, the amount of copies can be controlled.

There are a variety of ways in which gene expression can be evaluated. These include: Northern blots, Real-Time Polymerase Chain Reaction (Real-Time PCR), and microarray analysis. Northern blots use electrophoresis and a hybridization probe to measure mRNA levels. Northern blots generally provide qualitative or semiquantitative results of mRNA levels, they require relatively large amounts of RNA, and they only detect the expression levels for the gene specified by the probe. Real-Time PCR uses a DNA polymerase to amplify a piece of DNA by enzymatic replication, which is then detected via a fluorescence marker. Real-Time PCR can provide a quantitative measurement of mRNA, from a relatively low initial volume, however like northern blots, it is probe based, and so only the expression levels for the gene specified by the probe can be measured. RealTime PCR is often used to verify the expression of a limited number of genes found to be significant using microarray analysis.

Microarray analysis is a high-throughput technique that allows for the large-scale analysis and quantification of genes and gene expression. The microarrays consist of an ordered arrangement of sequences (oligonucleotides, cDNA, etc) attached, via covalent bonds, to the solid surface of a chemical matrix approximately $1.2 \mathrm{~cm} \times 1.2 \mathrm{~cm}$ in size. The protocol for the preparation of the microarrays using cDNA is as follows: 1) Isolate the RNA from the source material (cells, tissues, etc), 2) synthesize the total RNA to cDNA, 3) use the cDNA as a template to generate biotin-labeled in vitro transcribed cRNA, 4) fragment the cDNA and hybridize it to a microarray, 5) wash and stain the microarray with streptavidin phycoerythrin, and 6) analyze the microarray. Analysis is usually done using fluorescence that is created during the probe-target hybridization, and can determine the abundance of nucleic acid sequences in the target. Microarrays allow for tens-of-thousands of different genes to be analyzed at once from a single sample.

In the brain, Mahmoud-Ahmed et al [127] performed a comprehensive study of radiationinduced gene expression changes at different doses of radiation (2-, 10-, or 20-Gy) and at different time points (0.25-, 1-, 5-, and 24-hrs) for the 20-Gy dose group. They show that following 20-Gy, there are 78 genes that are modulated in the brain at 2 or more of the measured time points, with the maximum number of genes modulated at 1- and 5-hrs after radiation. In addition, they show that there are 24 biological pathways that are modulated by the 78 genes. At 5-hrs after radiation, where 2-, 10-, and 20-Gy analysis was performed, there was a dose-dependent biological response observed. In another study, Achanta et al used microarray analysis to measure gene expression in the hippocampus at 24-hrs following different doses of radiation (0.3-, 3-, 10-, and 30-Gy) as a first step to understanding radiation-induced cognitive decline. They showed a total of 1200 significant 
genes, however only 5 were observed across the different radiation doses. These 5 genes (MT-I, MT-II, Hsp-27, GFAP, and c-Foc) were then verified using RT-PCR. By using pathway analysis, the genes were shown to cluster around myc and tp53, genes that are known to be important in the regulation of the cell cycle and/or apoptosis.

To date, there are no long-term microarray studies following cranial radiation. Using radiation to the kidney or rectum, Kruse et al. evaluated radiation-induced gene expression changes at 10 and 20 weeks following a single dose of 16 (kidney)- or 20 (rectum)-Gy localized radiation. They reported that in the rectum there were 815 genes that were differentially expressed (278 up-regulated and 537 down-regulated) at 10 weeks, and 115 genes differentially expressed (86 up-regulated and 29 down-regulated) at 20 weeks. In the kidney, there were only 40 genes differentially expressed (31 up-regulated and 9 downregulated) at 10 weeks and 46 (42 up-regulated and 4 down-regulated) at 20 weeks. In both the rectum and kidney, there were very few genes that were common between the two time points, however there were genes related to vascular injury, in which they were actually interested in.

This work uses microarray analysis to create a gene profile of the acute damage following radiation to gain insight into the inflammatory, vascular and parenchymal response. The responses identified could then be explored as possible targets for interventional therapy in order to abrogate the late effects observed following cranial radiation. 


\section{Chapter 3}

\section{Materials and Methods}

\subsection{In vivo measurements}

\subsubsection{Animal model}

Male C57BL/6J mice (8-10 weeks of age) were purchased from Jackson Laboratories (Bar Harbor, ME). All protocols followed the policy guidelines of the National Institutes of Health for the humane care and use of laboratory animals and were approved by the Animal Care and Use Committees of St. Jude Children's Research Hospital and The University of Tennessee Health Science Center.

\subsubsection{Cranial window}

Cranial window surgery was performed according to a previously published protocol [128]. Briefly, animals were anesthetized with an i.m. injection of ketamine/xylazine solution $(100 \mathrm{mg} / \mathrm{kg}$ ketamine $+10 \mathrm{mg} / \mathrm{kg}$ xylazine), and placed in a stereotaxic frame (Kopf Instruments, Tujunga, CA). The scalp, underlying soft tissue, skull bone and dura were removed, and a glass cranial window $(4 \times 6 \mathrm{~mm})$, extending from the bregma to lambdoid sutures and centered on the midsagittal suture, was placed and fixed over the surgically exposed cerebral cortex using cyanoacrylate glue, Figure 3.1. After surgery, animals were allowed to recover for 7-10 days before data collection was initiated.

\subsubsection{Radiation treatment}

This study used a single dose of 20-Gy localized brain irradiation. The irradiator used was a cesium irradiator, Figure 3.2, at the Veterans Affairs Medical Center in Memphis Tennessee. While inside the irradiator chamber, the animals were anesthetized and secured to a platform using Velcro. All studies were done with localized cranial irradiation using a specially designed collimator (JL Shepard and associates, CA) allowing only a $1 \mathrm{~cm}$ wide field of irradiation (i.e., a $1 \mathrm{~cm}$ wide strip of radiation). From previous experiments studying localized radiation, this amount of radiation does not cause impairment to the animals. The irradiation field was confined to the brain with adequate lead shielding to ensure that tissue outside the field received less than $3 \%$ of the prescribed dose. 


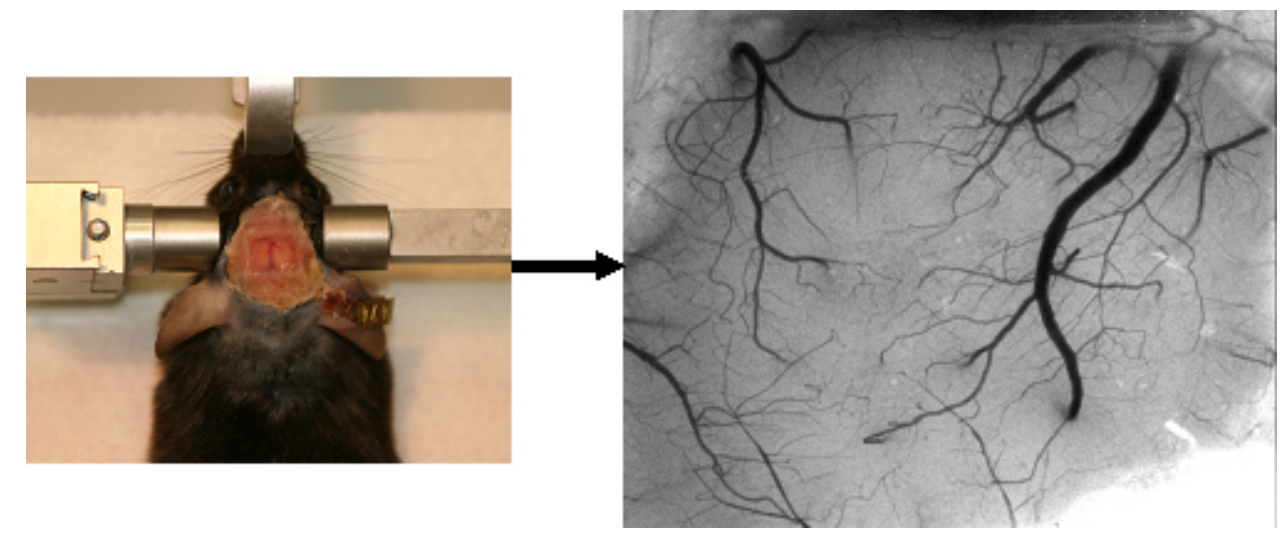

Figure 3.1: Picture of a mouse after placement of the cranial window.

A mouse with a cranial window in place. The window provides access to the vasculature of the pial layer of the brain. By using the intravital microscope and fluorescent dyes, we visualized changes.

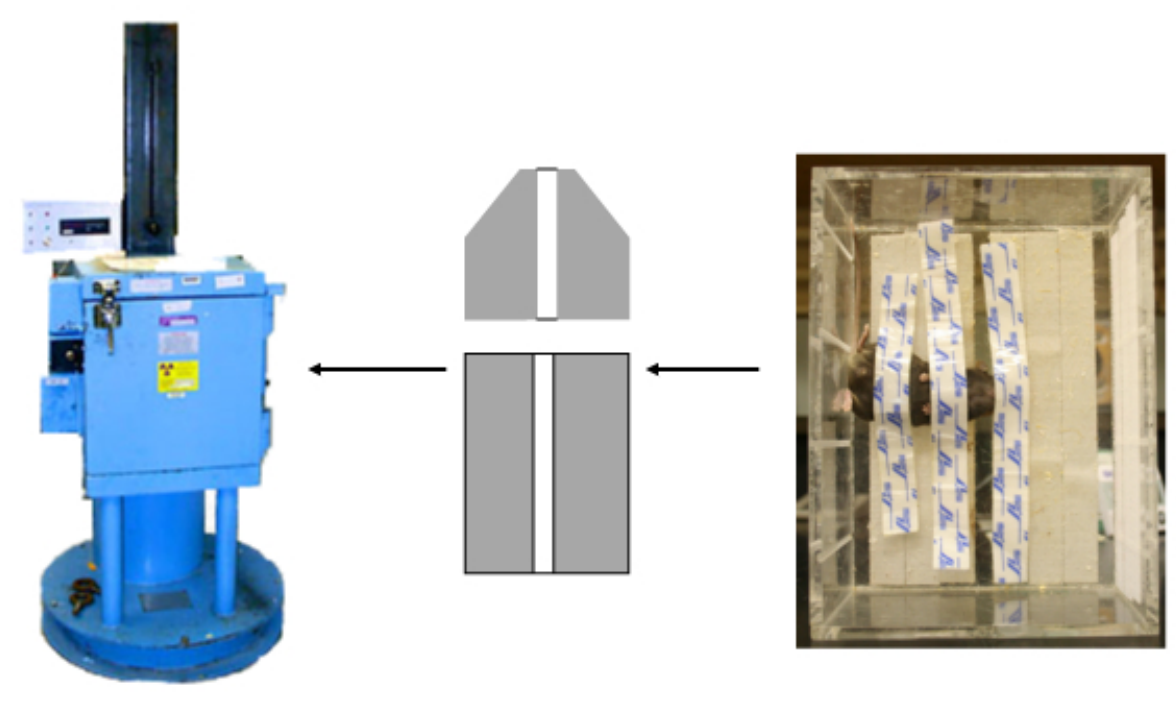

Figure 3.2: Irradiation set-up. Animals are irradiated using a cesium 137 source. There is a specially designed lead collimator inserted into the radiation chamber that limits exposure to a $1 \mathrm{~cm}$ wide strip field. Animals are placed in a specially-designed plexi-glass platform, in a horizontal position, such that only the cerebrum of the brain is exposed. The rest of the body is sheilded and receives less than $3 \%$ of the prescribed dose. 


\subsubsection{Anti-inflammatory treatments}

Animals receiving an antibody treatment were administered either purified rat antimouse TNF mAb (MP6-XT3), purchased from Pharmingen (San Diego, CA, catalog \#554414) or purified rat anti-mouse ICAM-1 mAb (YN1/1.7.4) purchased from eBioscience (San Diego, CA, catalog \#16-0541). These antibodies are used to react with mouse TNF and ICAM-1 expressed on the endothelial cell surface. Two doses of anti-TNF or antiICAM-1 were given to the mice: one dose $(2.0 \mathrm{mg} / \mathrm{kg}$, i.v.) at 15 minutes before irradiation, and another dose $(2.0 \mathrm{mg} / \mathrm{kg}$, i.v.) at 23 hours after irradiation. Purified rat IgG, purchased from Pharmingen (San Diego, CA, catalog \#554682), was used as a control for the specific antibodies.

KZ-41 was obtained from Dr Ryan Yates and Dr Duane Miller from the pharmaceutical science department at the University of Tennessee Health Science Center. Animals receiving KZ-41 received an i.p. injection at 30 minutes prior to irradiation and at 23- and 47-hrs after irradiation. KZ-41 was administered at a dose of $100 \mathrm{mg} / \mathrm{kg}$.

\subsubsection{Recombinant TNF}

To confirm the role of TNF in the response to irradiation, $0.5 \mu \mathrm{g}$ recombinant TNF (rTNF $\alpha$ BD Pharmingen, San Jose, CA; cat \# 554589) was administered via retro-orbital injection in mice with cranial windows. Measurements of leukocyte adhesion and vessel diameter were made as described above using Rhodamine-6G. Activated astrocytes were also measured. Measurements were made before injection and at 7- and 24-hrs postinjection of rTNF. In addition, mice treated with rTNF were analyzed for activated astrocytes at 7- and 24-hrs post-injection or rTNF.

\subsubsection{Intravital microscopy and BBB permeability}

The BBB permeability and leukocyte adhesion measurements using intravital microscopy, Figure 3.3, have been described in detail previously [128, 9]. Briefly, to visualize cerebral microcirculation, animals were anesthetized with ketamine-xylazine as described above and placed in a stereotaxic frame under a fluorescence intravital microscope. Fluorescein isothiocyanate (FITC)-dextran (Sigma, St. Louis, MO) with a molecular size of 4.4-kDa was used as a permeability tracer. Before dye injection, a microvascular region with 2-3 venules, arterioles, and clear parenchymal tissue was chosen and a reference image (for localization of vessels) recorded under epifluorescence illumination was acquired. A bolus of FITC-dextran in saline $(10 \mathrm{mg} / \mathrm{kg}$ body weight) was injected through the retro-orbital vein. The changes in intensity levels in the brain were digitally recorded after injection every second for the first 2 minutes, then for 5 seconds every 30 seconds for 5 minutes and then for 5 seconds every minute for up to 20 minutes. Figure 3.4 is an example of the extravasation of the FITC-dextran over a period of 5 minutes. This procedure was designed to minimize fluorophore excitation. All Images were made using an industrial-scale intravital microscope, Figure 3.3, (model NM-11, Nikon USA, Melville, NY) with a digital camera (Photometric CoolSnap ES, Roper Scientific, Trenton, NJ) and 


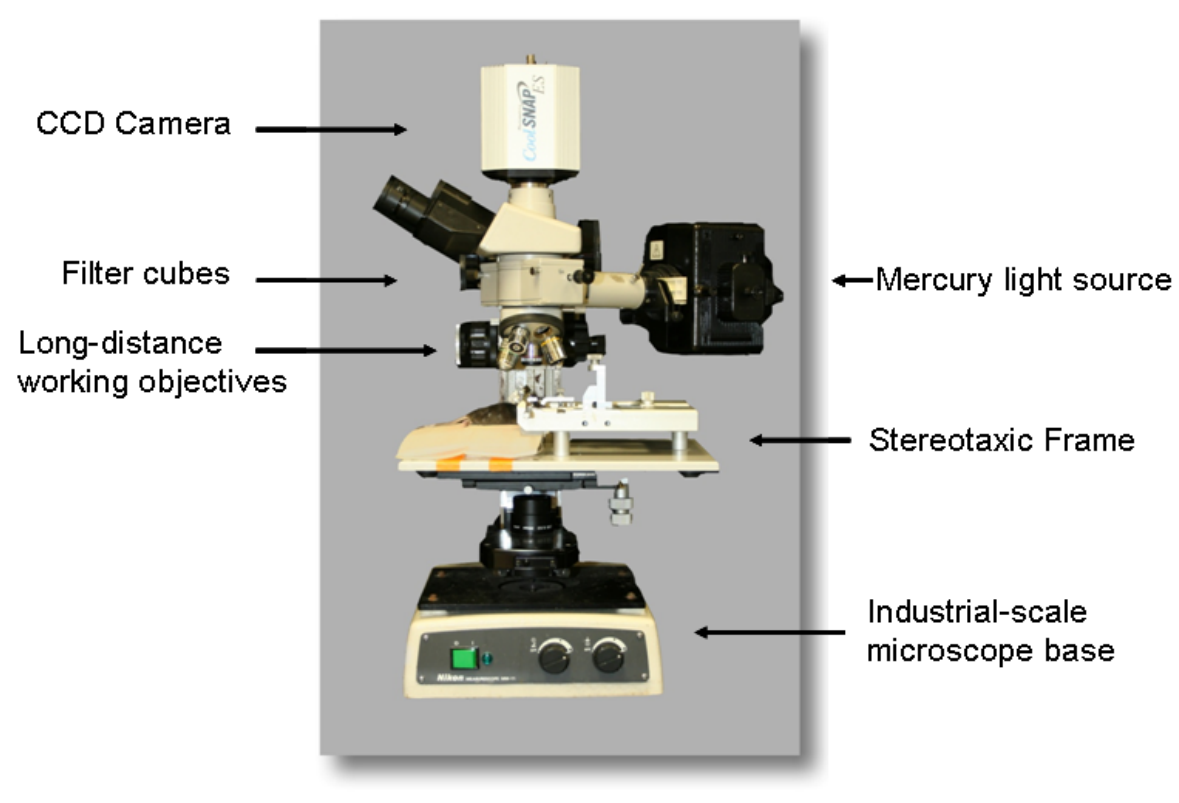

Figure 3.3: A picture of the intravital microscopy set-up.

The intravital microscope setup consists of an industrial scale microscope base designed to support the weight of a stereotaxic frame, used to minimize movement by the animal. There are long-distance working objectives that capture the fluorescent images. The working distance of the objectives allows us to focus on the surface of the brain through the glass window without being directly on the glass. Filter cubes control the wavelength of light that is allowed to pass to the subject for excitation and to the CCD camera as emission. The CCD camera is controlled by MetaMorph Imaging software which is used to capture the digital images and perform off-line analysis. 

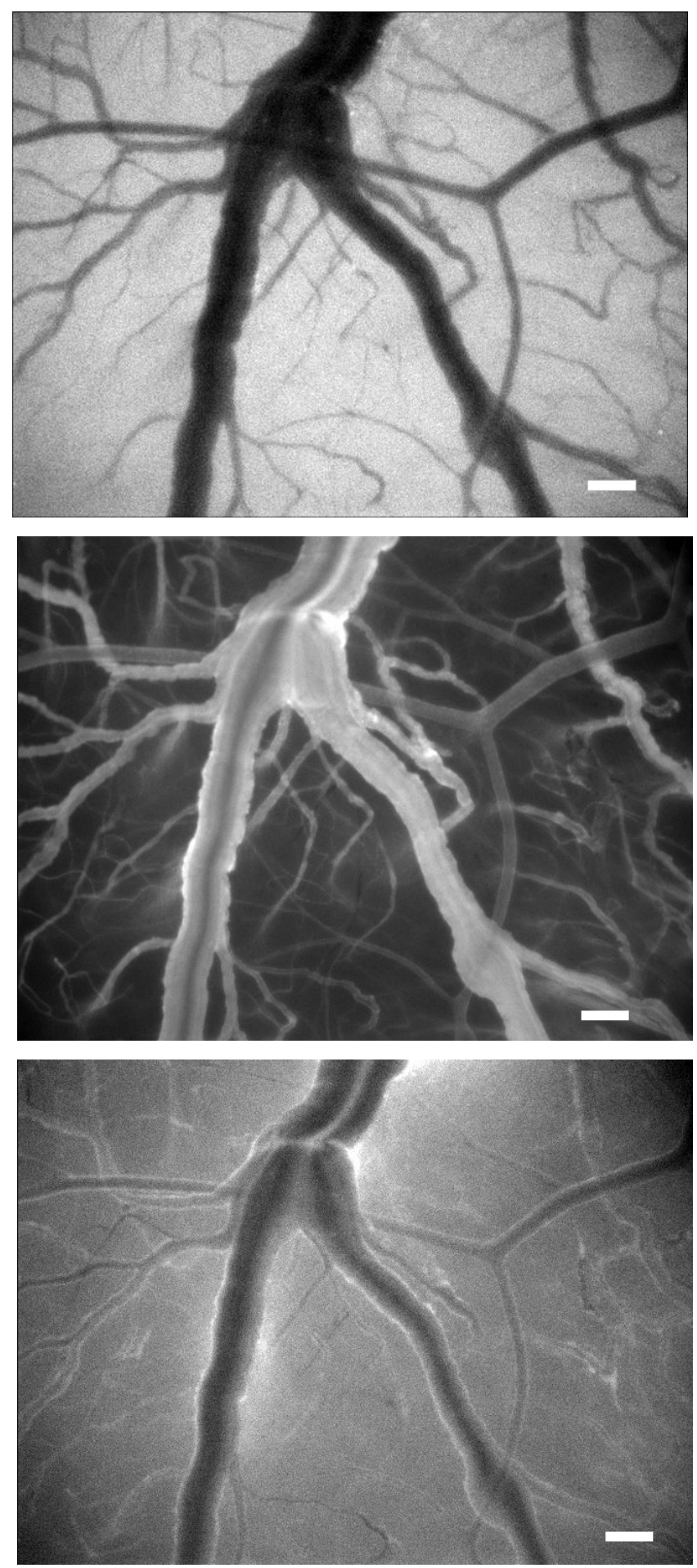

Figure 3.4: FITC-dextran 4.4kDa injection.

Visually, the extravasation of dye into the tissue is indicative of barrier permeability, the more dye that accumulates in the tissue the more permeable the BBB is. This series of images is an example of the data captured for analysis of BBB permeability to radiation. Upper: prior to injection, Center: peak intensity after injection of 4.4 kDa FITC-dextran, and Lower: 5 minutes post-injection. The images shown were taken at 24-hrs after irradiation where BBB permeability known to be increased. 
bright field (175 W mercury) light source. Images were analyzed off-line using MetaMorph software (Universal Imaging Co., West Chester, PA) to measure the extravasation rate of FITC-dextran from vessels to tissue, which is proportional to the slope of the change of tissue intensity versus time [128].

To calculate BBB permeability, two rectangular regions of interest (ROI) are chosen in the vessel and in the surrounding tissue and their average intensity is recorded over time, Figure 3.5. Our analysis method follows closely those reported by Wu et al [129], and Yuan et al [130], with some modifications. For slow extravasation processes, Fick's law can be used to show that the permeability $(P)$ and the rate of change of the tissue intensity due to dye extravasation $(d l / d t)$ are related [130].

$$
P=\left(1-H_{c t}\right) \frac{1}{l_{v}} * \frac{V}{S} *\left(\frac{d l_{i n t}}{d t}-\frac{d l_{S V}}{d t}\right)
$$

$I_{t}$ is the tissue intensity, $I_{V}$ is the initial fluorescence intensity in the vessel, $\mathrm{V} / \mathrm{s}$ is the ratio of vessel volume and surface area, and $H_{c t}$ is the local hematocrit and is set at its normal value of $32 \%[131,83]$. We limit our calculation of $\left(d l_{\text {int }} / d t-d l_{s v} / d t\right)$ to the time period between $30 \mathrm{~s}$ and $5 \mathrm{~min}$ after tracer injection to ensure that the dye is completely mixed. During this period, the extravasation process was approximately linear, and back flux of the tracer into the plasma compartment is secondary to the primary signal being measured. It is important to note that this is a $2 \mathrm{D}$ analysis of a 3D process.

Previous work by Hong Yuan [132], showed that there is a linear relationship, in vitro and in vivo, between dye concentration and emitted fluorescence intensity. This relationship was maintained in the current work. In addition, the depth of field for the objective used for in vivo measurements was calculated to be $10.4 \mu \mathrm{m}$, with a working distance of $16.5 \mathrm{~mm}$ at a resolution of $0.92 \mu \mathrm{m}$. The resolution of the camera used is $1300 \times 1030$ with $6.7 \times 6.7 \mu \mathrm{m}$ pixels.

\subsubsection{Leukocyte-endothelial interactions}

Leukocyte adhesion was measured by injecting Rhodamine-6G $(0.4 \mathrm{mg} / \mathrm{kg}$ body weight; Sigma, St. Louis, MO) through the retro-orbital vein to stain the leukocytes. Rhodamine-6G is a lipophilic, positively charged fluorescent dye that selectively stains mitochondria, and achieves a 99.99\% staining of leukocytes up to 5 minutes after injection at the concentration that was used in this study [133]. A leukocyte in contact with the vessel wall was considered to be adherent if it did not move for 30 seconds; this measure is expressed as the number of adherent cells per $100-\mu \mathrm{m}$ length of vessel [128]. The measurements were conducted in venules with diameters ranging from 30 to $45 \mu \mathrm{m}$. Permeability and adhesion measurements were carried out in the same vessels $(n=3-5)$ for each animal at each time point, before and after irradiation.

\subsubsection{Vessel diameter measurements}

The diameter of the arterioles and venules were measured in all animal groups (Figure 3.6). Diameters were measured 15 seconds after the peak of the FITC-dextran injection.

This time point ensured that the dye filled the vessels so that the full diameter could be 


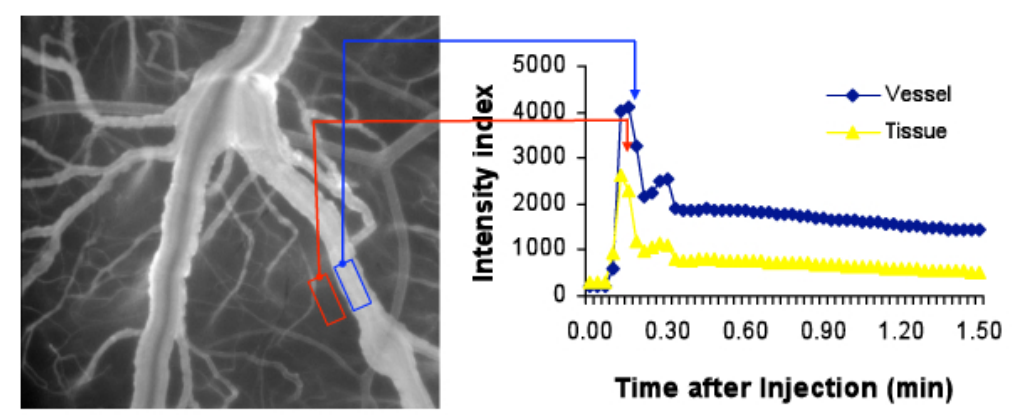

Figure 3.5: Permeability measurement.

When $4.4 \mathrm{kDa}$ FITC-dextran is injected, a region of interest is drawn in the vessel and tissue next to the vessel. Then, the average intensity of the two regions is recorded over time. The slope of the difference between the two regions is used as the rate of extravasation for the permeability calculation.

measured but was far enough from the peak to avoid light scatter in the measurement. Diameter measurements were made using digital calipers that had been calibrated. The calibration was done by determining the number of pixels on the image in a known distance using a micrometer. A calibration was done for each objective that was used. Measurements were carried out in the same vessels $(n=4-6)$ for each animal at each time point before and after radiation.

\subsubsection{Statistics}

Statistical analyses were carried out using SigmaStat ${ }^{\circledR}$ software (version 2.03, SPSS Science, Chicago, IL). The effects of radiation on BBB permeability and leukocyte adhesion were determined by repeated analysis of variance (ANOVA), and a multiple comparison procedure (Dunnett test) was used to discriminate among the means. Results of the effects of radiation on BBB permeability and leukocyte adhesion between treatment groups were compared using the student t-test. Data are presented as mean $\pm \mathrm{SEM}$, and differences between the means were considered to be statistically significant at the probability value $P<0.05$. Linear regression analysis was used to investigate the correlation between BBB permeability and leukocyte adhesion measured in vivo in the same animals. Leukocyte adhesion was set as the dependent variable and BBB permeability as the independent variable. The coefficient of determination, $r^{2}$, was used to asses the strength of the model as it describes the fraction of the observed variance that can be explained by the given variables. The correlation coeficient can be obtained by taking the square root of $r^{2}$, and represents the strength and direction of the linear relationship between between BBB permeability and leukocyte adhesion. 

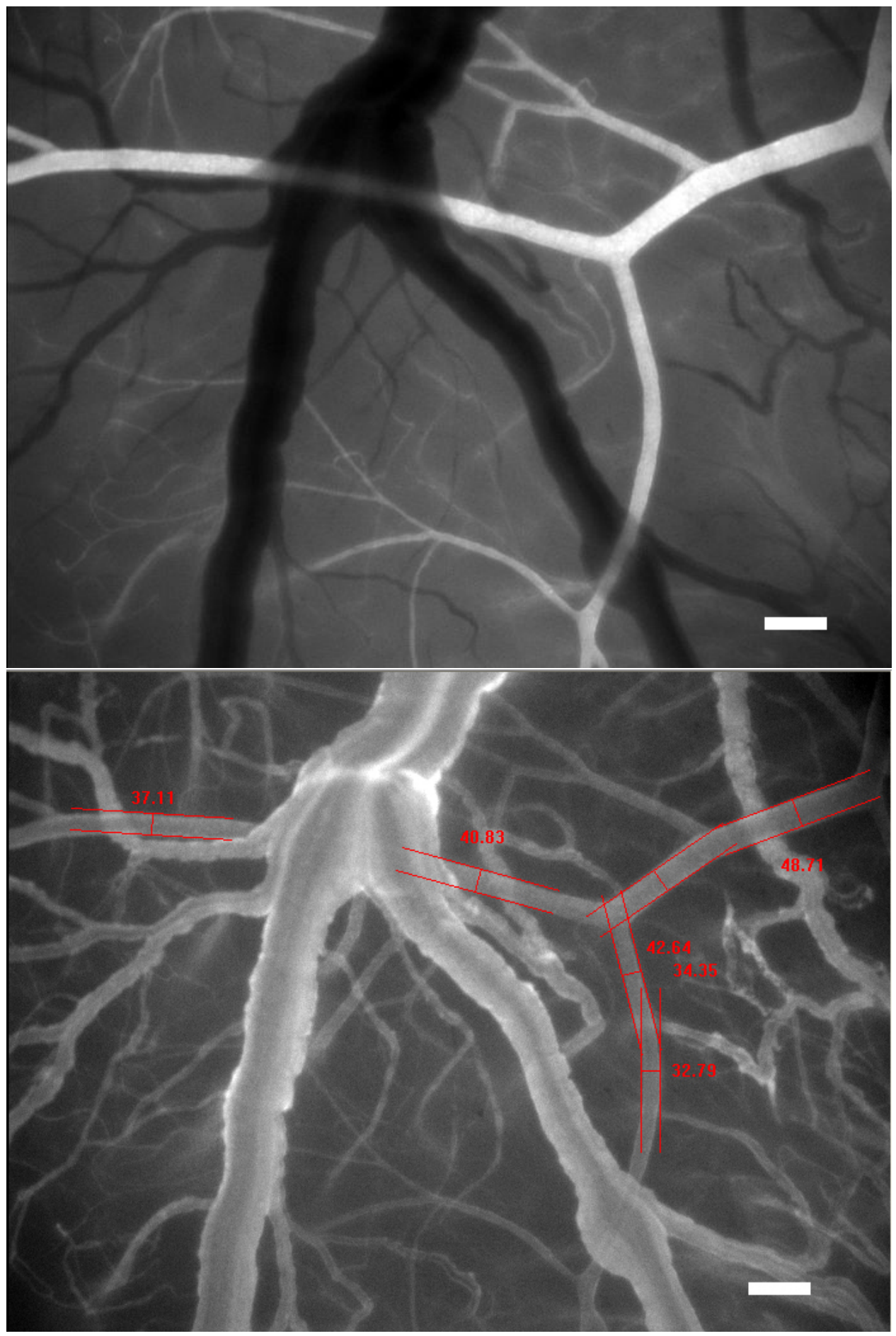

Figure 3.6: Vessel diameter measurement.

Arteries are identified by their smooth surface, as compared to the venules, and at time of injection. Dyes are injected via the retro-orbital vein, so in the vasculature measured in the cranial window, the dye fills the arteries first (Left) and then enters the venules. At approximately 15 seconds after the peak of the injected dye, calibrated calipers are used to measure at multiple locations along vessels to obtain an average vessel diameter (Right). 


\subsection{Histological measurements}

\subsubsection{Tissue collection}

Anesthetized mice were perfused through the left ventricle of the heart with heparinized saline $(0.9 \%)$. Following perfusion, whole brain tissue was removed and sectioned at the center in the coronal plane. The anterior portion was used for immunofluorescence staining and was snap frozen in Tissue-Tek O.C.T. Compound (Fisher Scientific, Pittsburgh, PA) in liquid nitrogen. The posterior portion was used for immunohistochemistry studies and placed in $10 \%$ buffered formalin overnight prior to embedding in paraffin.

\subsubsection{Immunohistological staining}

Glial fibrillary acidic protein (GFAP) was used to stain for activated astrocytes. Rabbit anti-cow GFAP (cat \# N1506), and rabbit immunoglobulins (negative control, cat \# X0903) were purchased from DAKO (Carpinteria, CA). The secondary antibody for GFAP, biotinylated goat anti-rabbit, were purchased from Vector (cat \# BA-1000, Burlingame, CA) and used at a 1:200 dilution. Immunohistochemistry assays were performed on the DAKO autostainer at room temperature. The number of GFAP-stained astrocytes were counted in five fields at 200x magnification in the cerebral cortex overlying the dentate gyrus of the hippocampus, and in the hippocampus itself using a light microscope, Figure 3.7. Each image presented is representative of $n=3$ animals.

\subsubsection{Immunofluorescence staining}

Frozen brains will be sectioned at $7 \mu \mathrm{m}$ using a cryostat and mounted on poly-1-lyisnecoated glass slides. Slides will be incubated with; goat anti-mouse TNF (Cat\# sc-1350) or goat anti-mouse ICAM-1 (Cat\# Sc-1511) antibody at a 1:100 dilution for 60min, followed by a fluorescence-conjugated secondary antibody for $45 \mathrm{~min}$. All antibodies and mounting medium were purchased from Santa Cruz Biotechnology, Santa Cruz CA. The sections were analyzed for protein expression at 400x magnification in the cerebral cortex overlying the dentate gyrus of the hippocampus, and in the hippocampus itself, Figure 3.7. These areas were chosen because they are identifiable which ensures that similar locations are evaluated in all sections. Each image presented is representative of $n=3$ animals.

\subsubsection{Statistics}

Statistical analyses were carried out using SigmaStat® software (version 2.03, SPSS Science, Chicago, IL). Results of the effects of radiation on astrogliosis between treatment groups were compared using the student t-test. Data are presented as mean $\pm \mathrm{SEM}$, and differences between the means were considered to be statistically significant at the probability value $\mathrm{P}<0.05$. 


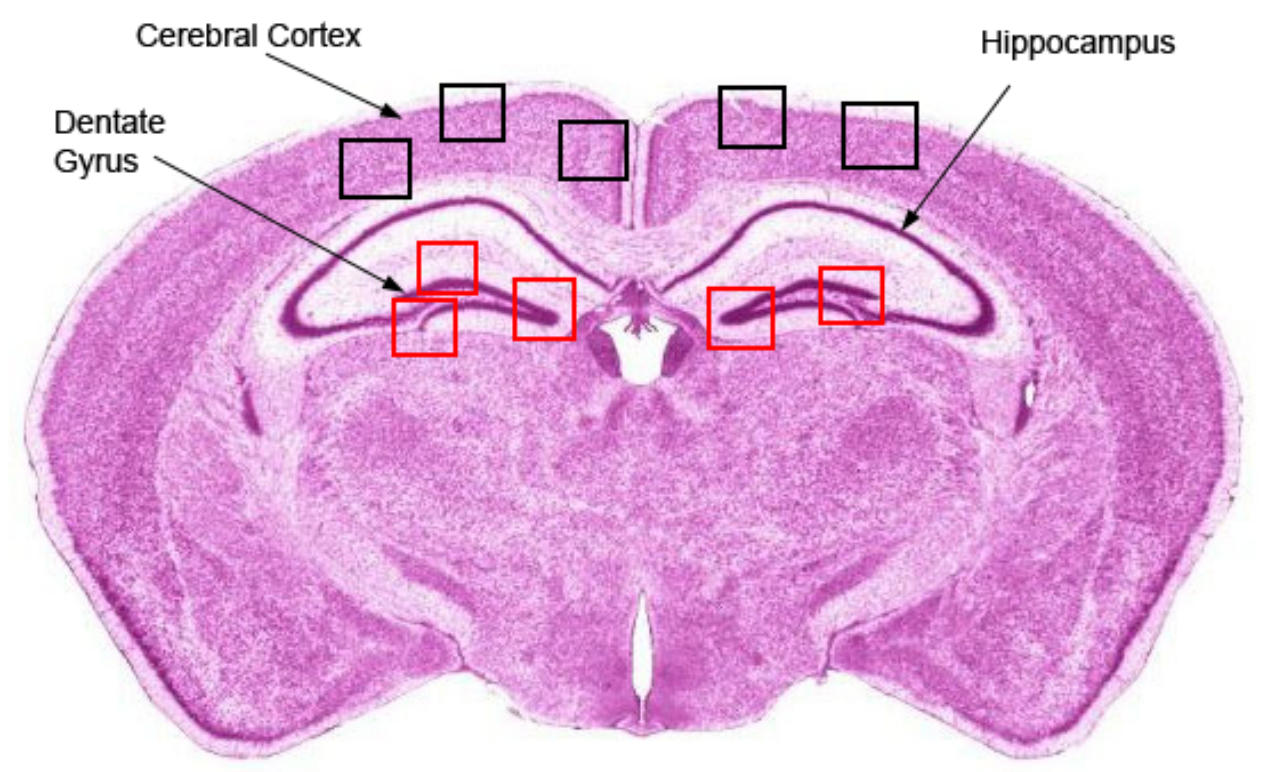

Figure 3.7: Regions used for histological analysis.

All measurements for both immunohistochemistry and immunofluorescence were made in the cerebral cortex overlying the dentate gyrus of the hippocampus. The activated astrocytes were also counted in the dentate gyrus region to confirm that the changes in astrocyte activation in the cerebral cortex represented changes in other regions of the brain. The black boxes represent areas that were analyzed in the cerebral cortex, and red boxes represent area that were analyzed in the denate gyrus region. 


\subsection{Microarray experiments}

\subsubsection{Tissue collection}

Animals received a single dose of 20-Gy localized brain irradiation. Mice were sacrificed at 2-hrs after a single dose of irradiation. Upon sacrifice, whole brains were removed and snap frozen in liquid nitrogen. Brains were stored in a $-80^{\circ} \mathrm{C}$ freezer until analyzed. A total of six samples were collected at each time point. The five samples with the purist RNA were chosen to use on the microarray chips.

\subsubsection{RNA preparation}

For RNA extraction, frozen whole mouse brain (300 - $500 \mathrm{mg}$ ) tissue was added to $5 \mathrm{ml}$ of STAT-60 (Tel-Test, TX), homogenized, using a Polytron homogeninizer and stored on ice for $10 \mathrm{~min}$. Chloroform/isoamyl (24:1, v:/v) ETOH was added to the homogenate and centrifuged at $12000 \mathrm{~g}$ for $30 \mathrm{~min}\left(4^{\circ} \mathrm{C}\right)$. Following centrifugation, the aqueous phase was transferred to a fresh tube, RNA was precipitated with isopropanol overnight at $-20^{\circ} \mathrm{C}$, pelleted by centrifugation at $12000 \mathrm{~g}$ for $30 \mathrm{~min}\left(4^{\circ} \mathrm{C}\right)$, and resuspended $100 \mu \mathrm{l}$ of RNasefree water. Total RNA was quantified using a NanoDrop Spectrophotometer (NanoDrop Technologies, Rockland, DE), and qualified by running $1 \mu \mathrm{l}$ of each sample onto a RNA Lab-On-A-Chip using a Agilent Bioanalyzer 2100 (Agilent technologies, Mountain View, $\mathrm{CA})$.

\subsubsection{Chip preparation}

Isolated total RNA samples were processed as recommended by Affymetrix (Affymetrix GeneChip Expression Analysis Technical Manual, Affymetrix, Inc.). Briefly, total RNA (8 mg) was synthesized to cDNA using the Superscript Double-Stranded cDNA synthesis kit (Invitrogen Corp, Carlsbad, CA) and poly dT-nucleotide primers that contain a sequence recognized by T7 RNA polymerase. The newly synthesized cDNA was used as a template to generate biotin-labeled invitro transcribed (IVT) CRNA using the Bio-Array High Yield RNA transcript labeling kit (Enzo Diagnostics, Inc, Farmingdale, NY). Twenty micrograms of the cRNA was fragmented to strands of 35 to 200 bases in length. The fragment cRNA was hybridized to and Affymetrix MOE 4302.0 array GeneChip at $45^{\circ} \mathrm{C}$ with rotation for $16 \mathrm{~h}$ (Affymetrix GeneChip Hybridization Oven 320). The GeneChip arrays were washed and stained (streptavidin phycoerythrin) on an Affymetrix Fluidics Station 400, followed by scanning. Chips were analyzed using an Affymetrix GC 3000 System.

\subsubsection{Chip analysis}

The Entrez Gene ID's of the statistically significant genes were uploaded as a list to Webgestalt [134]. Webgestalt is a "WEB-based GEne SeT AnaLysis Toolkit" that is available for free online through Vanderbilt University (http://bioinfo.vanderbilt.edu/webgestalt/). 
One of the many functions of Webgestalt is gene set organization and visualization that includes: KEGG Tables and Maps, GOTree's, Tissue Expression Bar Charts, BioCarta Table and Maps, and Protein Domain Tables. For the current study, KEGG Tables and Maps were utilized in order to organize the data. The KEGG Table organizes the genes from the given list based on the known KEGG biochemical pathways. The KEGG Table provides the KEGG pathways associated with the gene set, the total number of genes in each pathway present in the given gene set, and the Entrez Gene IDs for the genes in the set. Once Webgestalt generated the KEGG Table, the pathways present in the table were then manually grouped by molecular interaction and reaction networks.

\subsubsection{Statistics}

Data output from MAS 5.0 (Affymetrix) was analyzed using GeneSpring7.3.1 (Agilent Technologies). A parametric student t-test was used to identify the genes that were significantly differentially expressed. In order to reduce the variations in sample loading and staining, expression values for each probe set were normalized to the median expression value (50th percentile) for all probe sets in each chip. When doing PCA or making histogram boxplot graphs, each gene was normalized to the median expression value across the set of microarrays that were analyzed.

Before any analysis was performed, the data were filtered by flags (absent $(A)$, present(P) or marginal(M) detection calls) and expression values. The genes were kept if their flag values were present or marginal and the expression values above 40 of the raw level in at least 3 out of 5 samples.

The average of all five microarray chips for each time point were used to determine statistical analysis and for pathway analysis. The differences between the means were considered to be statistically significant at the probability value $\mathrm{P}<0.05$.

\subsection{Serum cytokines}

Serum cytokine measurements were made in mice following cranial irradiation. Mice were irradiated using the procedure above, and sacrificed at 24-, 48-, 72-hrs and 8- and 15-days post-RT. Mice were anesthetized with isoflurane, followed by a cardiac puncture to obtain a blood sample. Approximately 0.5 to $0.8 \mathrm{mls}$ of blood was collected. The sample was allowed to coagulate for 10 minutes at room temperature before being placed on ice for up to 30 minutes. The sample was then centrifuged for $2 \times 10$ minutes. The serum was then removed and stored in microtubes at $-80^{\circ} \mathrm{C}$ until analysis. Analysis was done using a FlowMetrixTM system (Luminex, Austin, TX) run by the Mouse Immunophenotyping and Cytokine Assay Lab in the Immunology department at St. Jude Children's Research Hospital. The cytokines that were analyzed are TNF, VEGF, IL-6, IL-10, and GCSF. The statistical analysis of the serum cytokines was done using a mixed model method. 


\section{Chapter 4}

\section{Mechanism of Radiation-Induced Inflammation}

It is accepted that patients who receive cranial irradiation for treatment of a brain tumor will incurr some side effects of treatment. These side effects generally do not appear until months to years following treatment. However, in the field of radiation research, the cause for these side effects is often debated. Currently, it is not accepted that: there is a relation between acute damage and late-term damage, the importance of the vasculature in radiation damage (early or late-term), and that radiation induces inflammation in the brain. The current work aims o show that radiation induces damage to the vasculature and parenchyma and that his damage is due to the inflammatory response that is initiated following radiation.

Hypothesis: Brain irradiation causes an acute response which if unchecked creates a chronic inflammatory microenvironment which amplifies radiation sequela. Treatment with anti-TNF or anti-ICAM-1 agents during the early phase arrests this inflammatory response and reduces radiation toxicity of the normal brain tissue.

Specific Aim: Characterize the acute damage to the blood-brain barrier, following a single dose of 20-Gy localized cranial irradiation (RT) using a mouse cranial window model and histological methods and evaluate the effect of treatment with anti-TNF or anti-ICAM-1 in conjunction with irradiation to abrogate inflammation-induced radiation damage.

The specific questions being investigated are:

- What is the role of TNF and ICAM-1 in the acute changes observed following radiation?

- Does inhibiting TNF affect the levels of ICAM-1 and vise versa?

- Is there a correlation between the increase in vascular permeability and leukocyte adhesion? Does blocking either molecule, TNF or ICAM-1, disrupt this correlation?

\subsection{Radiation-induced changes in the permeability of the BBB}

Changes in BBB permeability to 4.4-kDa FITC-dextran were measured before and at 24- and 48-hrs post-radiation (Figure 4.1). Prior to radiation, BBB permeability was 13.14 $\pm 1.99 \times 10^{-7} \mathrm{~cm} / \mathrm{s}$. At 24 - and 48-hrs post-irradiation permeability significantly increased to $20.30 \pm 2.36 \times 10^{-7} \mathrm{~cm} / \mathrm{s}(P<0.05)$ and $21.53 \pm 3.61 \times 10^{-7} \mathrm{~cm} / \mathrm{s}(P<0.05)$, respectively. When treated with radiation and anti-TNF, BBB permeability was significantly lower at 24- 


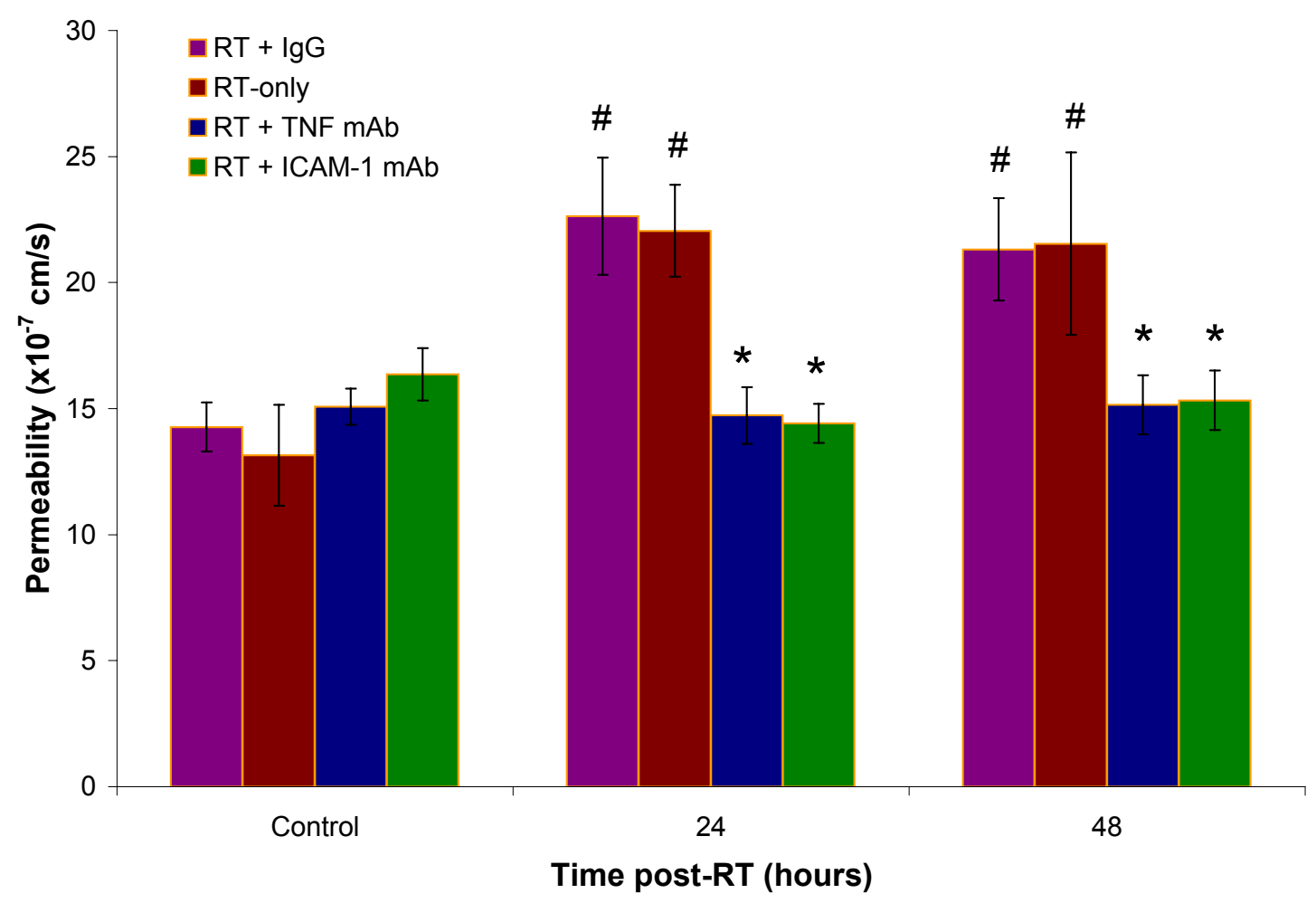

Figure 4.1: Vascular permeability of the BBB following irradiation.

Radiation induces an increase in vascular permeability of the BBB at 24- and 48-hrs following 20-Gy localized cranial irradiation. When the inflammatory response is limited via treatment with either TNF or ICAM-1 antibody, the increase in vascular permeability is inhibited. \# $\mathrm{P}<0.05$ versus Control, ${ }^{*} \mathrm{P}<0.05$ versus $\mathrm{RT}$-only at the given time point. 
and 48-hrs compared to the radiation-only group. The respective values were $14.73 \pm 1.12$ $\times 10^{-7} \mathrm{~cm} / \mathrm{s}(\mathrm{P}<0.05)$ and $15.14 \pm 1.17 \times 10^{-7} \mathrm{~cm} / \mathrm{s}(\mathrm{P}<0.05)$. Similarly, when treated with radiation and anti-ICAM-1 BBB permeability was significantly lower, $14.42 \pm 0.77 \times 10^{-7}$ $\mathrm{cm} / \mathrm{s}(\mathrm{P}<0.05)$ and $15.33 \pm 1.18 \times 10^{-7} \mathrm{~cm} / \mathrm{s}(\mathrm{P}<0.05)$ at $24-$ and 48-hrs post-irradiation, respectively. In the sham group ( $R T+\lg G$ administered), permeability was not significantly different that RT-only at any time point $\left(14.27 \pm 0.97 \times 10^{-7} \mathrm{~cm} / \mathrm{s}, 22.63 \pm 2.32 \times 10^{-7}\right.$ $\mathrm{cm} / \mathrm{s}, 21.32 \pm 2.03 \times 10^{-7} \mathrm{~cm} / \mathrm{s}$, at control, 24- and 48-hrs respectively).

\subsection{Radiation-induced leukocyte adhesion in the brain}

Radiation induced a significant increase in leukocyte adhesion in venules (Figures 4.2 and 4.3). Prior to radiation, leukocyte adhesion was $0.45 \pm 0.13$ adhering leukocytes $/ 100 \mu \mathrm{m}$ and was significantly increased compared to control at 24- and 48-hrs post-radiation: 1.01 $\pm 0.25(\mathrm{P}<0.05)$ and $1.02 \pm 0.15(\mathrm{P}<0.01)$, adhering leukocytes $/ 100 \mu \mathrm{m}$, respectively. When treated with radiation and anti-TNF, adhesion was significantly lower up to $48 \mathrm{hrs}$ post-radiation. Adhesion was $0.49 \pm 0.12(\mathrm{P}<0.05)$ and $0.44 \pm 0.12(\mathrm{P}<0.01)$, adhering leukocytes $/ 100 \mu \mathrm{m}$, at 24- and 48-hrs post-radiation, respectively. Similarly, when treated with radiation and anti-ICAM-1 the number of adhering leukocytes was significantly less at 24- $(0.46 \pm 0.17$ adhering leukocytes $/ 100 \mu \mathrm{m} ; \mathrm{P}<0.05)$ and 48 -hrs $(0.52 \pm 0.19$ adhering leukocytes $/ 100 \mu \mathrm{m} ; \mathrm{P}<0.05)$ compared to radiation-only. Treatment with radiation and IgG did not cause the number of adhering leukocytes to differ from radiation alone at any time point. There was no adhesion observed in arterioles in any group at any time point.

\subsection{Changes in pial vessel diameters following cranial irradiation}

In the radiation-only group, there was a significant decrease in arteriole diameter at 48-hrs post-irradiation ( $22.45 \pm 1.16 \mu \mathrm{m}$; $\mathrm{P}<0.05)$, compared to before radiation $(27.16 \pm$ $2.17 \mu \mathrm{m})$, Figure 4.4. When treated with radiation and anti-TNF there was no decrease in arteriole diameter, making the average arteriole diameter significantly larger at 48-hrs $(29.10 \pm 1.43 \mu \mathrm{m} ; \mathrm{P}<0.01)$ post-irradiation in the radiation and anti-TNF treated group than in the radiation-only group $(22.45 \pm 1.16 \mu \mathrm{m})$. Similarly, treatment with radiation and anti-ICAM-1 inhibited the loss in arteriole diameter at 48-hrs post-irradiation. The average arteriole diameter at 48-hrs in the radiation and ICAM-1 group was $(28.63 \pm 2.40 \mu \mathrm{m}$; $P<0.05)$. Animals treated with radiation and lgG exhibited the same decrease in arteriole diameter at 48-hrs post-irradiation $(28.29 \pm 1.12 \mu \mathrm{m} ; \mathrm{P}<0.05)$ as the radiation-only group. There was no significant change in the diameter of the venules in the radiation-only or in any treated group at any time point measured.

\subsection{Correlation between BBB permeability and leukocyte adhesion}

Leukocyte adhesion has been implicated in endothelial cell remodeling which, in turn, could lead to changes in vascular pore size and increases in permeability $[114,128]$. To 


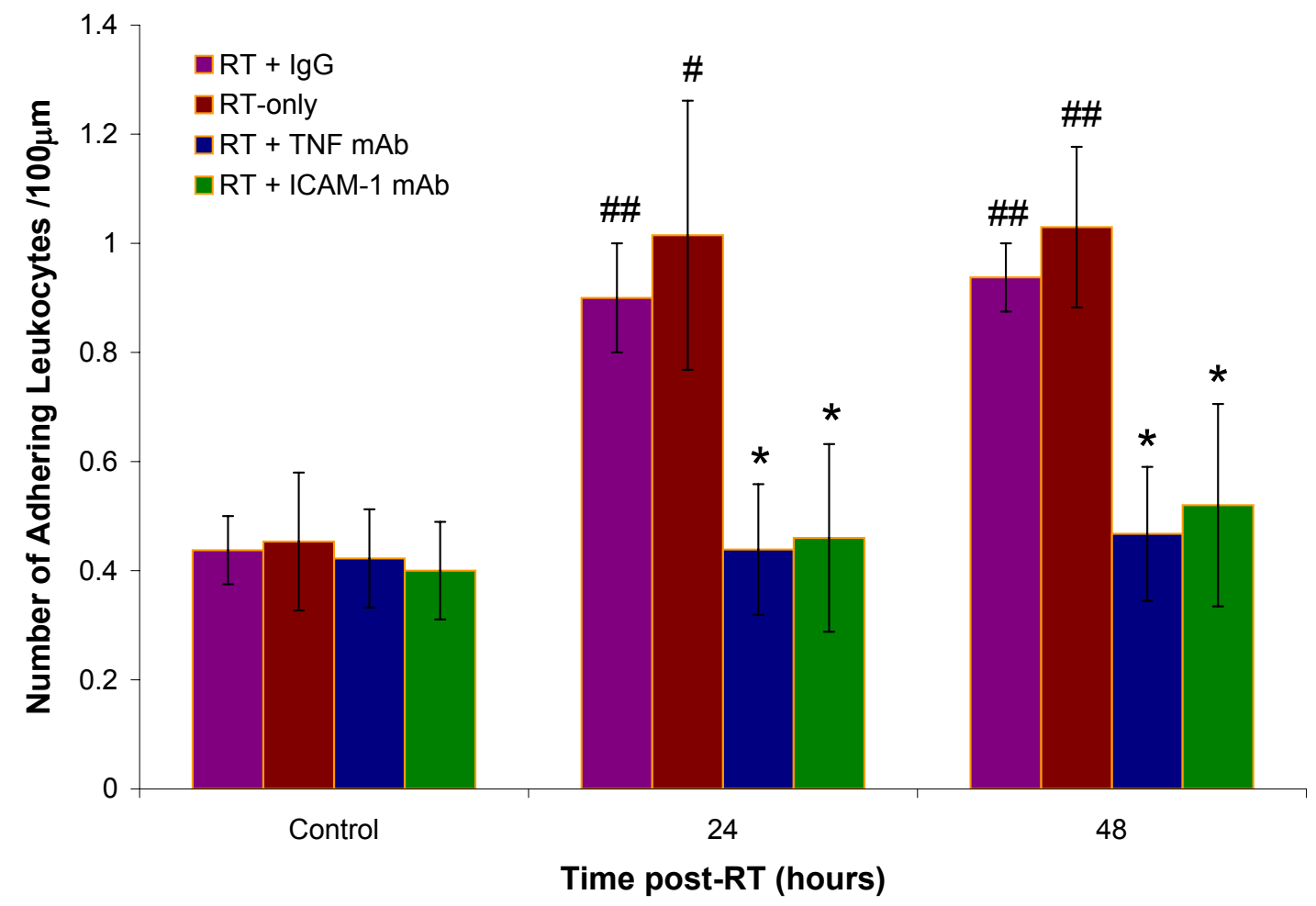

Figure 4.2: Leukocyte adhesion following irradiation.

Radiation induces an increase in adhering leukocytes in the pial vessels of the brain at 24and 48-hrs following 20-Gy localized cranial irradiation. When the inflammatory response is limited via treatment with either TNF or ICAM-1 antibody, the increase in adhering leukocytes is inhibited. \# $\mathrm{P}<0.05$, \#\# $\mathrm{P}<0.01$ versus control, ${ }^{*} \mathrm{P}<0.05$ versus $\mathrm{RT}$-only at the given time point. 

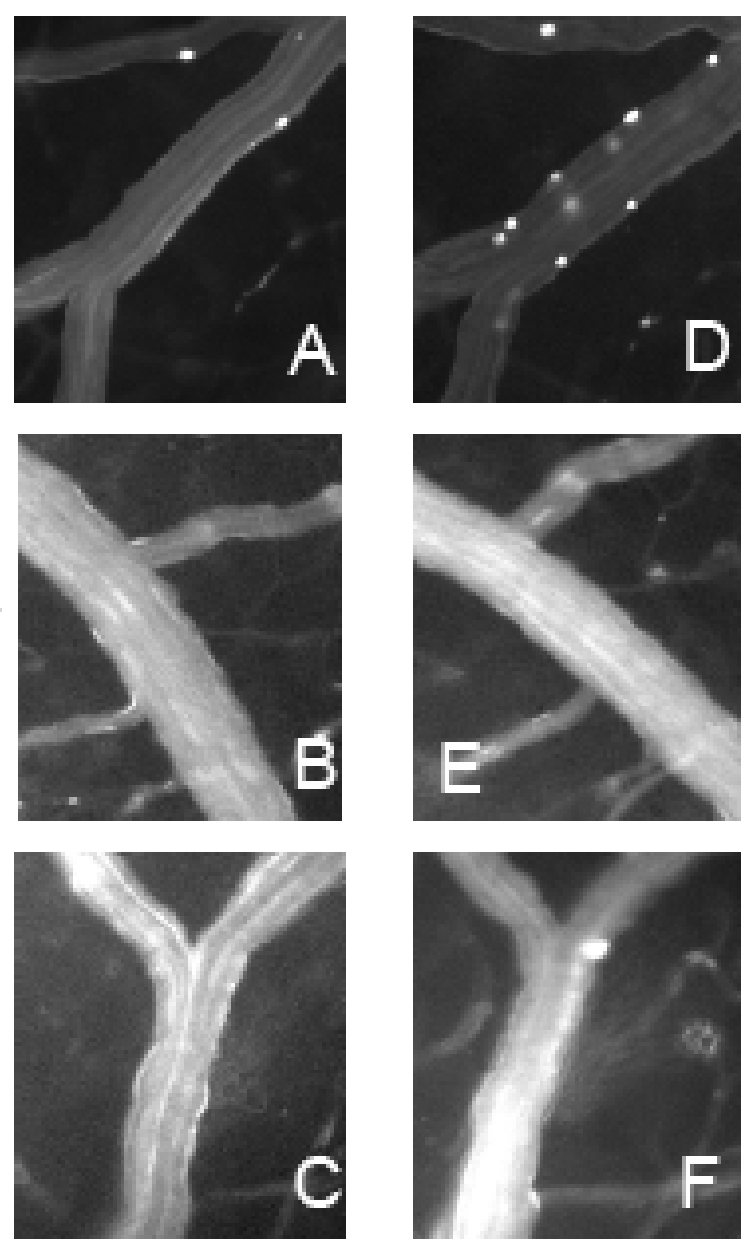

Figure 4.3: Representative images of radiation-induced leukocyte adhesion.

Representative images of the changes observed in leukocyte adhesion 24-hrs following RT. (A-C) Control images with few adherent leukocytes observed (D) 24-hrs post-20-Gy RT-only; a significant amount of adhering leukocytes are visible. (E) 24-hrs post-20-Gy $\mathrm{RT}+$ anti-TNF and (F) 24-hrs post-20-Gy RT + ICAM-1; when treated prior to irradiation, the adhering of leukocytes can be inhibited. Images were taken at approximately the same time after injection of Rhodamine-6G. 


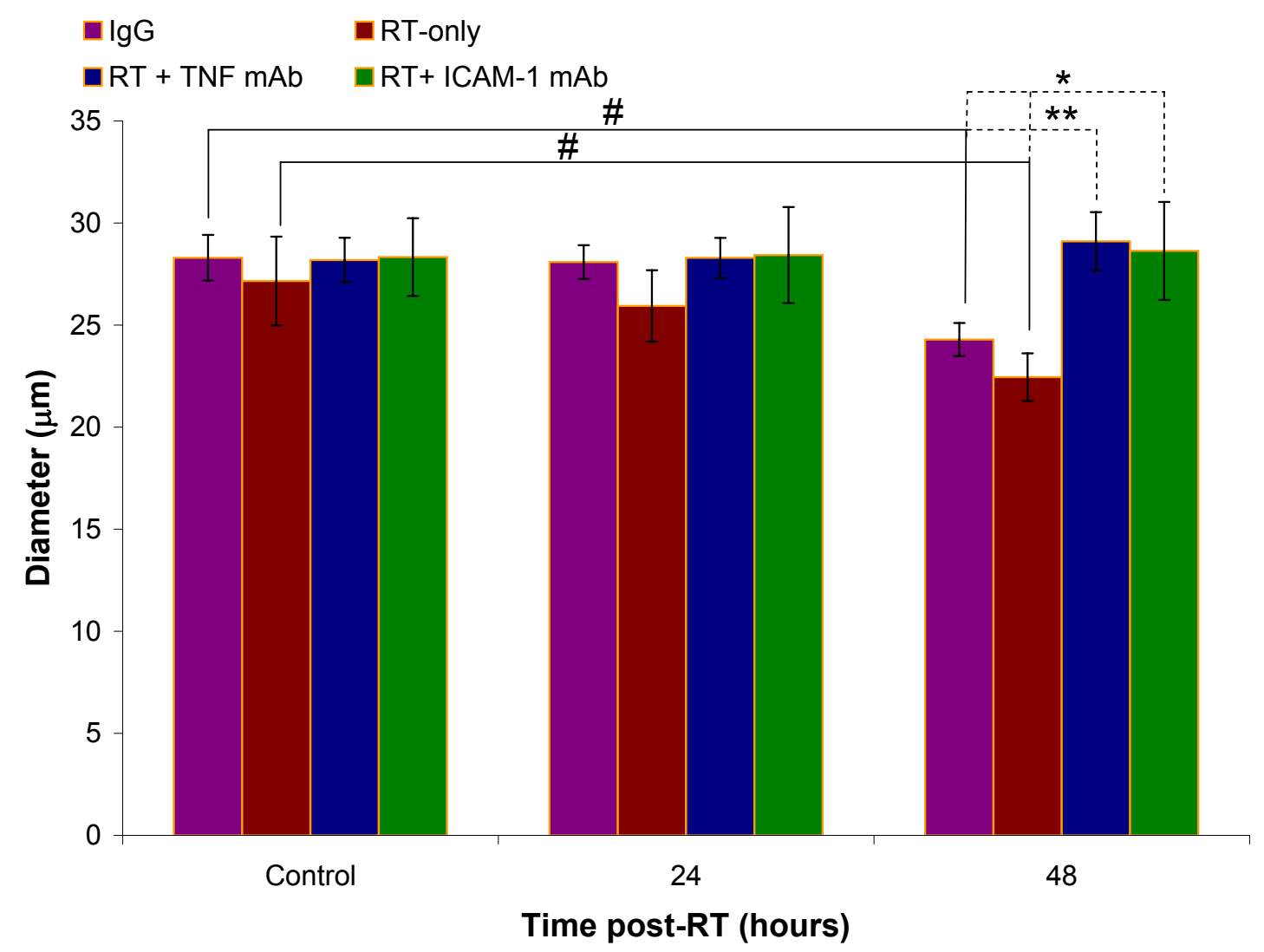

Figure 4.4: Radiation-induced changes in vessel diameter.

Radiation induces a decrease in arteriole vessel diameter at 48-hrs following a single dose of 20-Gy localized cranial radiation. This decrease in vessel diameter is inhibited when the inflammatory response is limited. There is a significant difference between both treated groups and radiation-only at 48 -hrs post-radiation. \# $\mathrm{P}<0.05$ versus Control, ${ }^{*} \mathrm{P}<0.05$ and ${ }^{* *} \mathrm{P}<0.01$ versus RT-only at the given time point. 
investigate this possible correlation a linear regression analysis was performed with leukocyte adhesion as the dependent variable. The analysis was performed using four sets of data: radiation-only, radiation-only and radiation and anti-TNF, radiation-only and radiation and anti-ICAM-1, and all three groups together, Figure 4.5. The results showed that the number of adhering leukocytes significantly influences BBB permeability in each data set analyzed. In the radiation-only group, there was a coefficient of determination of 0.8746 $(\mathrm{P}<0.001)$ and an increase in BBB permeability of $6.5021 \times 10^{-7} \mathrm{~cm} / \mathrm{s}$ for every adhering leukocyte in $100 \mu \mathrm{m}$ distance. Radiation-only and radiation and anti-TNF showed a coefficient of determination of $0.6499(\mathrm{P}<0.001)$ while radiation and radiation and anti-ICAM-1 showed a coefficient of determination of $0.6997(\mathrm{P}<0.001)$. When all three sets of data were combined, the coefficient of determination was 0.6365 with an increase in BBB permeability of $3.77 \times 10^{-7} \mathrm{~cm} / \mathrm{s}$ for every adhering leukocyte in $100 \mu \mathrm{m}$ distance.

\subsection{Immunohistochemistry}

There was a significant increase in the number of GFAP positive astrocytes in the cerebral cortex at 24- and 48-hrs post-radiation. In the unirradiated brain there were 11.41 \pm 1.21 activated astrocytes $/ \mathrm{mm}^{2}$, Figures 4.6 and 4.7 . At 24 - and 48 -hrs post-irradiation activated astrocyte counts were $21.92 \pm 0.24(\mathrm{P}<0.001)$ and $22.92 \pm 0.36(\mathrm{P}<0.001)$ activated astrocytes $/ \mathrm{mm}^{2}$, respectively. In the radiation and anti-TNF treated group the number of activated astrocytes was not significantly different than control at any time point measured. In the radiation and anti-ICAM-1 treated group, the number of activated astrocytes was significantly greater that in the unirradiated or radiation and anti-TNF treated groups, however it was still significantly less than in the radiation-only group. At 24- and 48-hrs postirradiation, the activated astrocyte counts, in the radiation and anti-ICAM-1 treated group, were $13.56 \pm 0.69$ activated astrocytes $/ \mathrm{mm}^{2}$ and $13.38 \pm 0.33$ activated astrocytes $/ \mathrm{mm}^{2}$, respectively.

To confirm that the observed increase, or inhibition, of activated astrocytes in the cerebral cortex is consistent in other regions of the brain, activated astrocytes were counted in the dentate gyrus region of the hippocampus. The astrocytic response in the dentate gyrus region mimicked the response in the cerebral cortex, Figure 4.8. The only difference was in the number of activated astrocytes. In the cerebral cortex, the activated astrocyte counts ranged from $11.41 \pm 1.21$ activated astrocytes $/ \mathrm{mm}^{2}$ in the unirradiated to 34.38 \pm 0.41 activated astrocytes $/ \mathrm{mm}^{2}$, while in the dentate gyrus region, the activated astrocyte counts ranged from $27.57 \pm 1.83$ activated astrocytes $/ \mathrm{mm}^{2}$ to $66.26 \pm 2.05$ activated astrocytes $/ \mathrm{mm}^{2}$.

In the cerebral cortex, astrocyte activation was significantly increased in the radiation and IgG group compared to the unirradiated group at 24- and 48-hrs post-irradiation, and not significantly different from RT-only, $27.58 \pm 0.2$ activated astrocytes $/ \mathrm{mm}^{2}$ and 26.03 \pm 1.11 activated astrocytes $/ \mathrm{mm}^{2}$, respectively. Similarly, in the dentate gyrus region of the hippocampus, the number of activated astrocytes in the radiation and IgG group was not different than the radiation-only group at 24- and 48-hrs post-irradiation (65.39 \pm 1.46 activated astrocytes $/ \mathrm{mm}^{2}$ and $66.56 \pm 1.88$ activated astrocytes $/ \mathrm{mm}^{2}$, respectively). 
Figure 4.5: Correlation between BBB permeability and leukocyte adhesion.

Using linear regression analysis, we can see that there is a good correlation between permeability of the BBB and leukocyte adhesion. 


\section{RT-only}

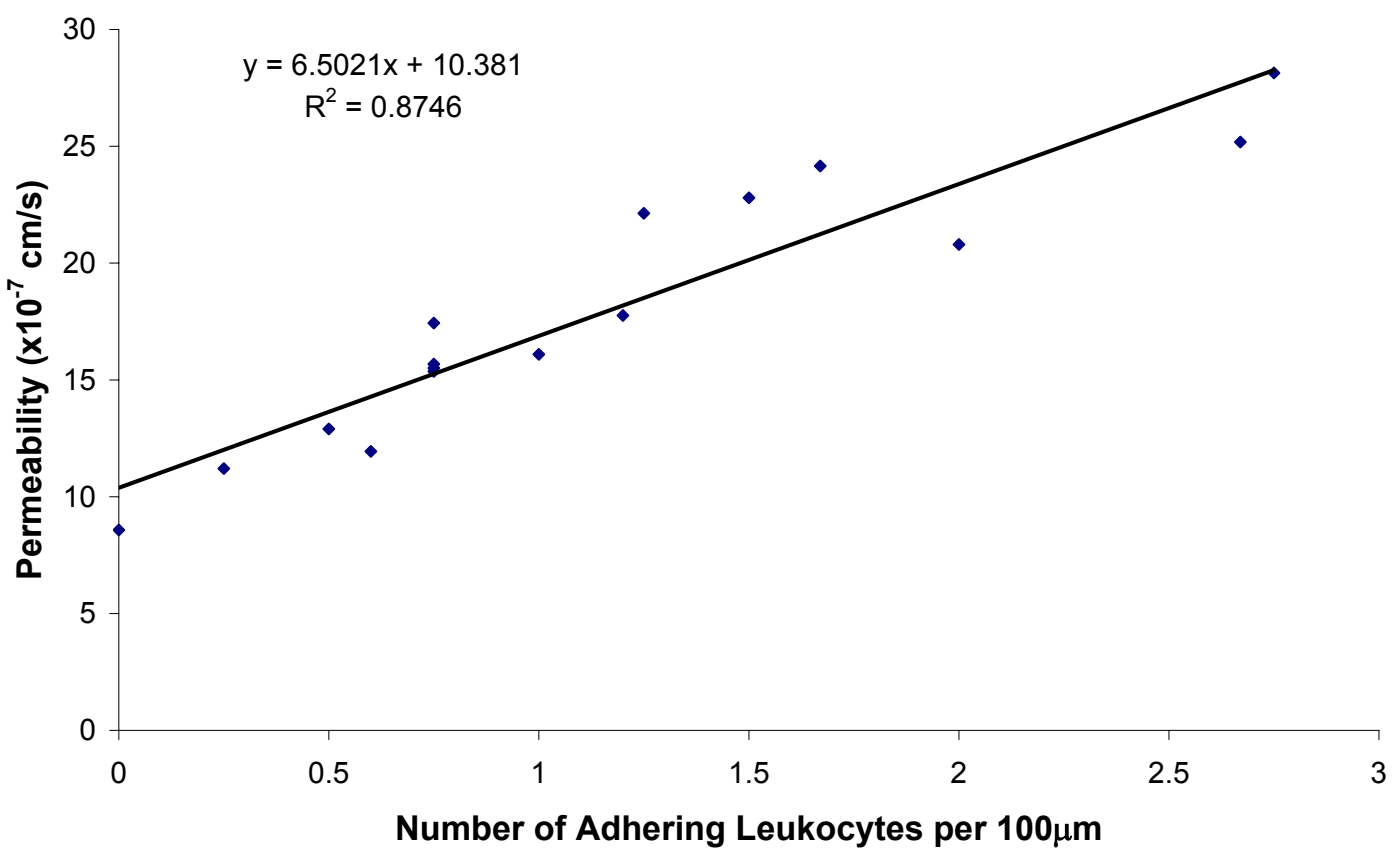

RT-only and RT + TNF mAb

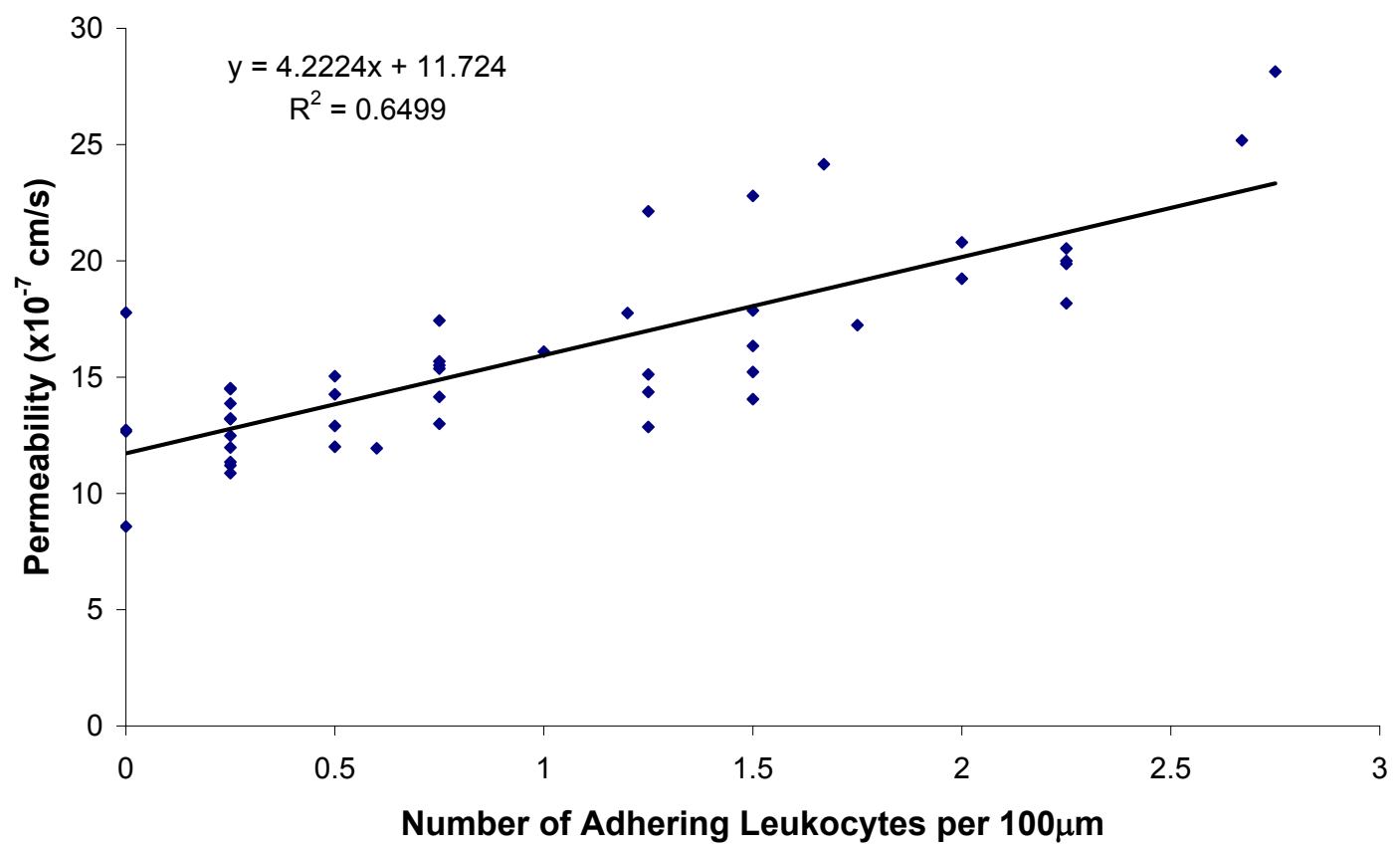




\section{RT-only and RT + ICAM-1 mAb}

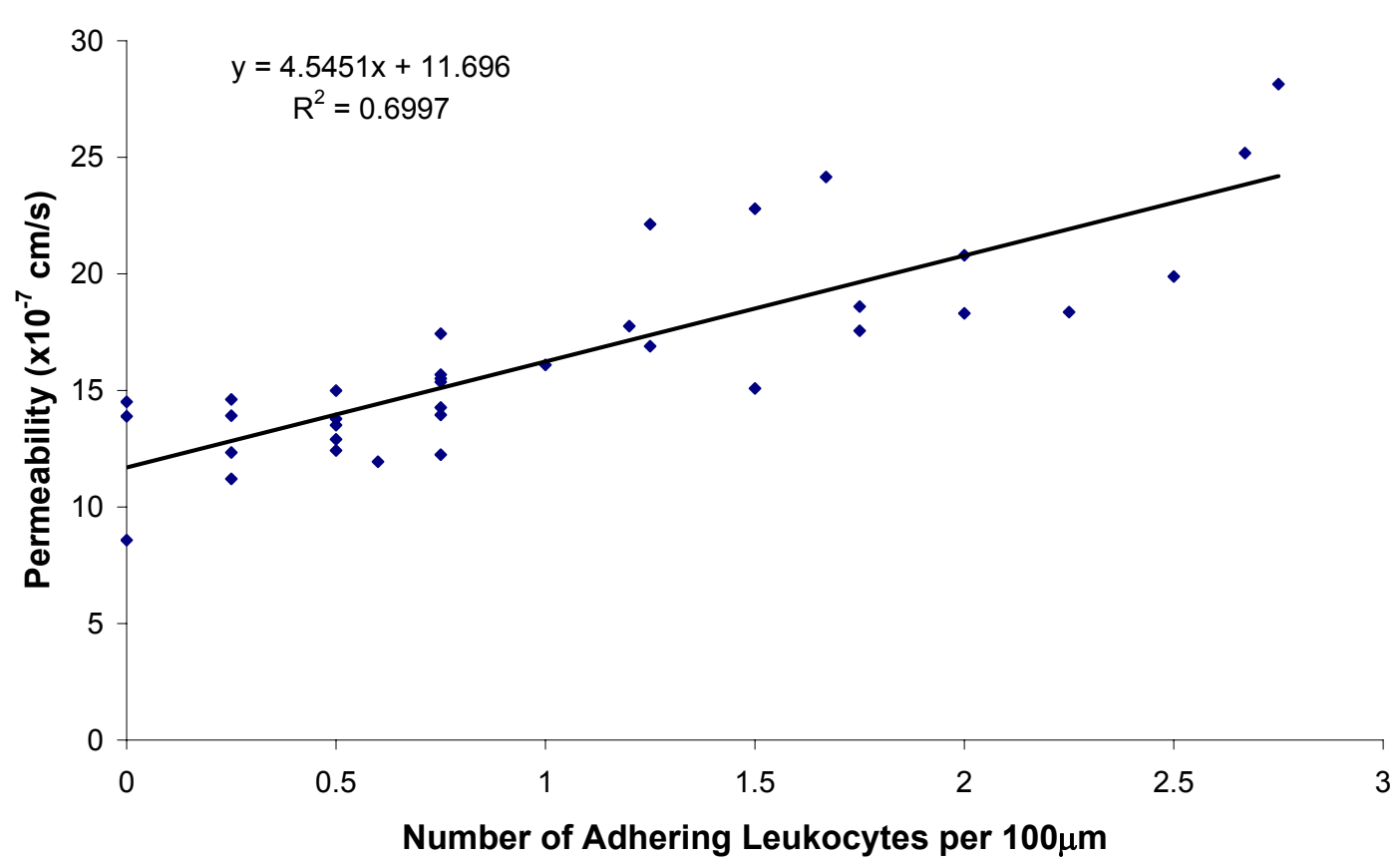

RT-only, RT + TNF mAb, RT + ICAM-1 mAb

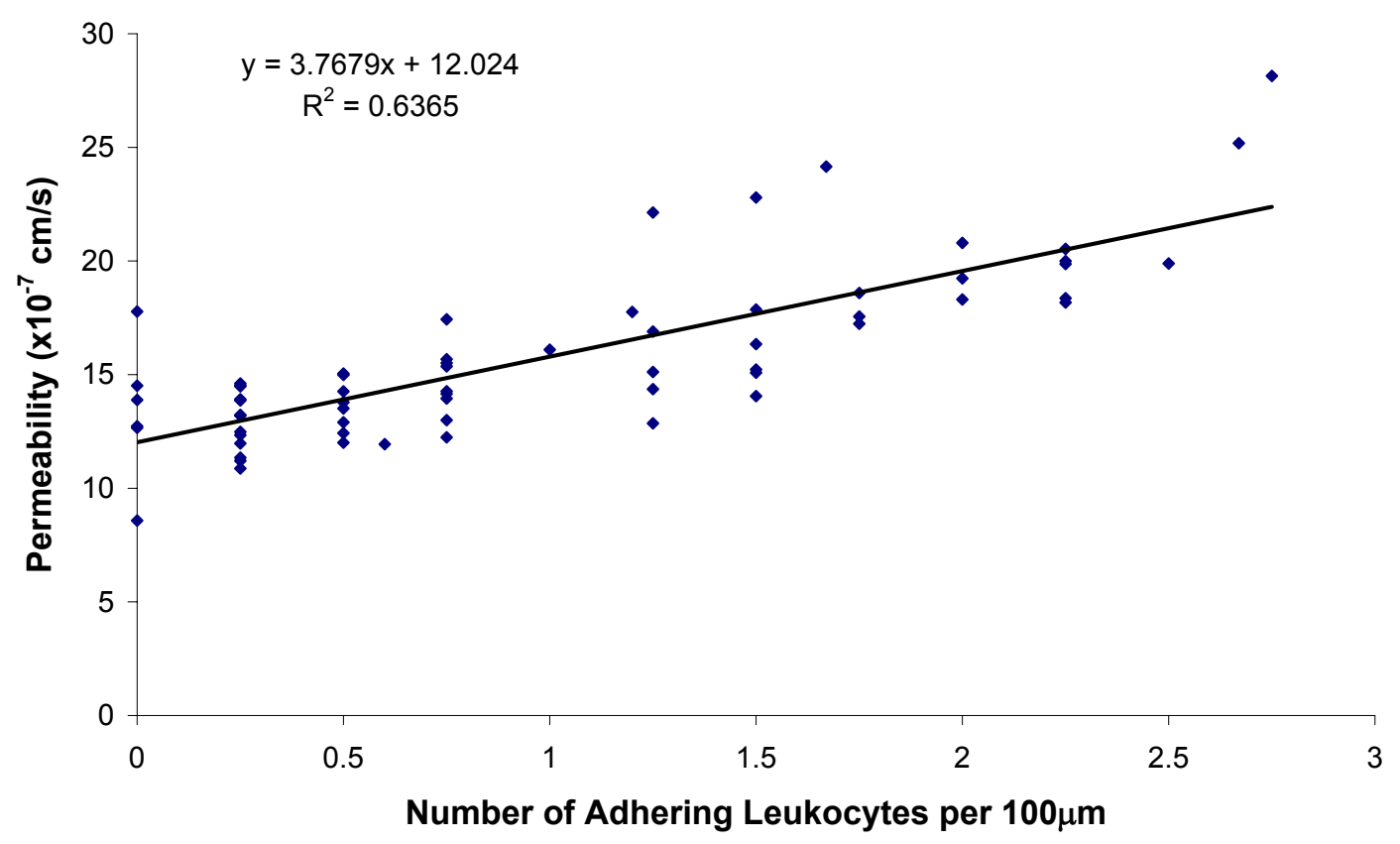

Figure 4.5: (continued). 


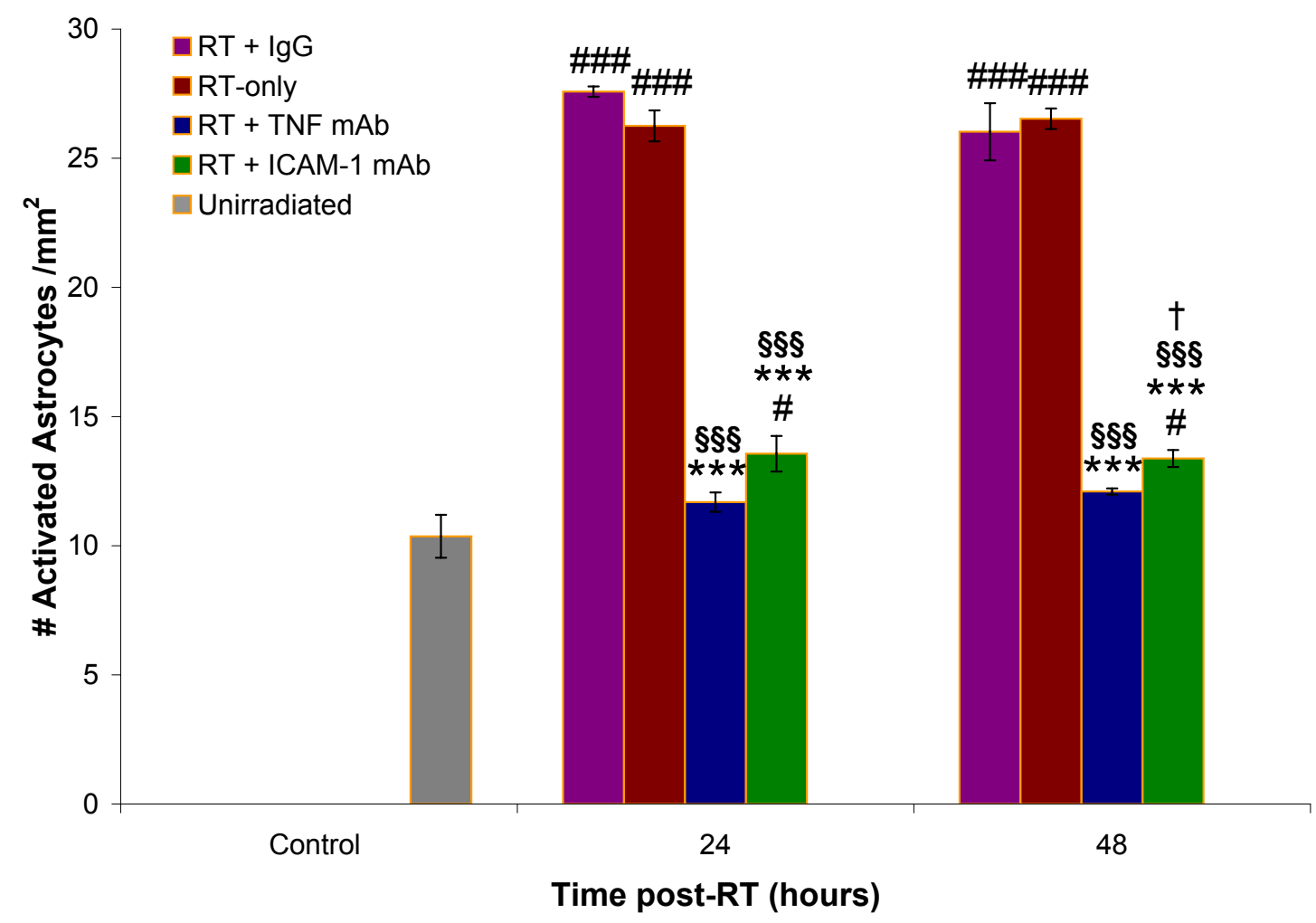

Figure 4.6: Activated astrocyte counts in the cerebral cortex.

Following RT, a significant amount of astrogliosis was observed at 24- and 48-hrs and was significantly inhibited when treated with anti-TNF or anti-ICAM-1. Treatment with radiation and IgG did not effect activated astrocyte levels when compared to radiation only. $(P<0.05)$ and ${ }^{* * *}(P<0.001)$ indicate a significant difference versus control. \#\#\# $(P<0.001)$ indicates a significant difference between the radiation and anti-TNF or radiation and antiICAM-1 treated group and the RT-only group at that time point. $\dagger(P<0.05)$ indicates a significant difference between the radiation and anti-TNF treated group and the radiation and anti-ICAM-1 treated group at that time-point. $\S \S \S(P<0.001)$ indicates a significant difference versus radiation and IgG treatment at that time point. 

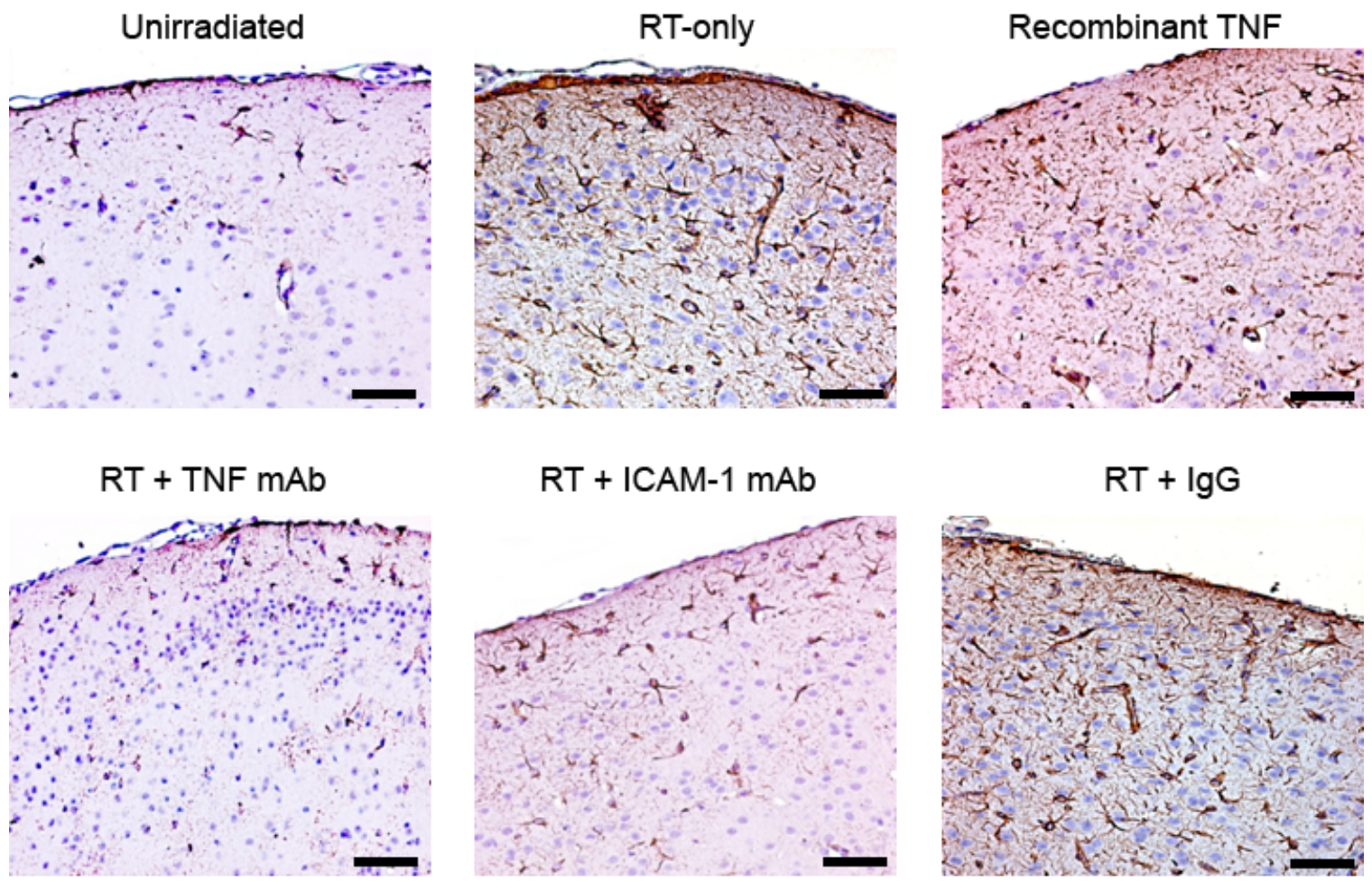

Figure 4.7: GFAP staining for activated astrocytes.

Representative images of GFAP staining at 24-hrs post-radiation (24-hrs post-injection for the recombinant TNF). There is a significant difference in activated astrocytes in the RT-only brain, RT + IgG and the rTNF brain compared to the unirradiated and the RT + TNF $\mathrm{mAb}$, and RT + ICAM-1 mAb treated brains. Images were taken at the same exposure settings and scaled the same. Magnification 200x. Each image presented is representative of $n=3$ animals. Scale bar $=100 \mu \mathrm{m}$ 


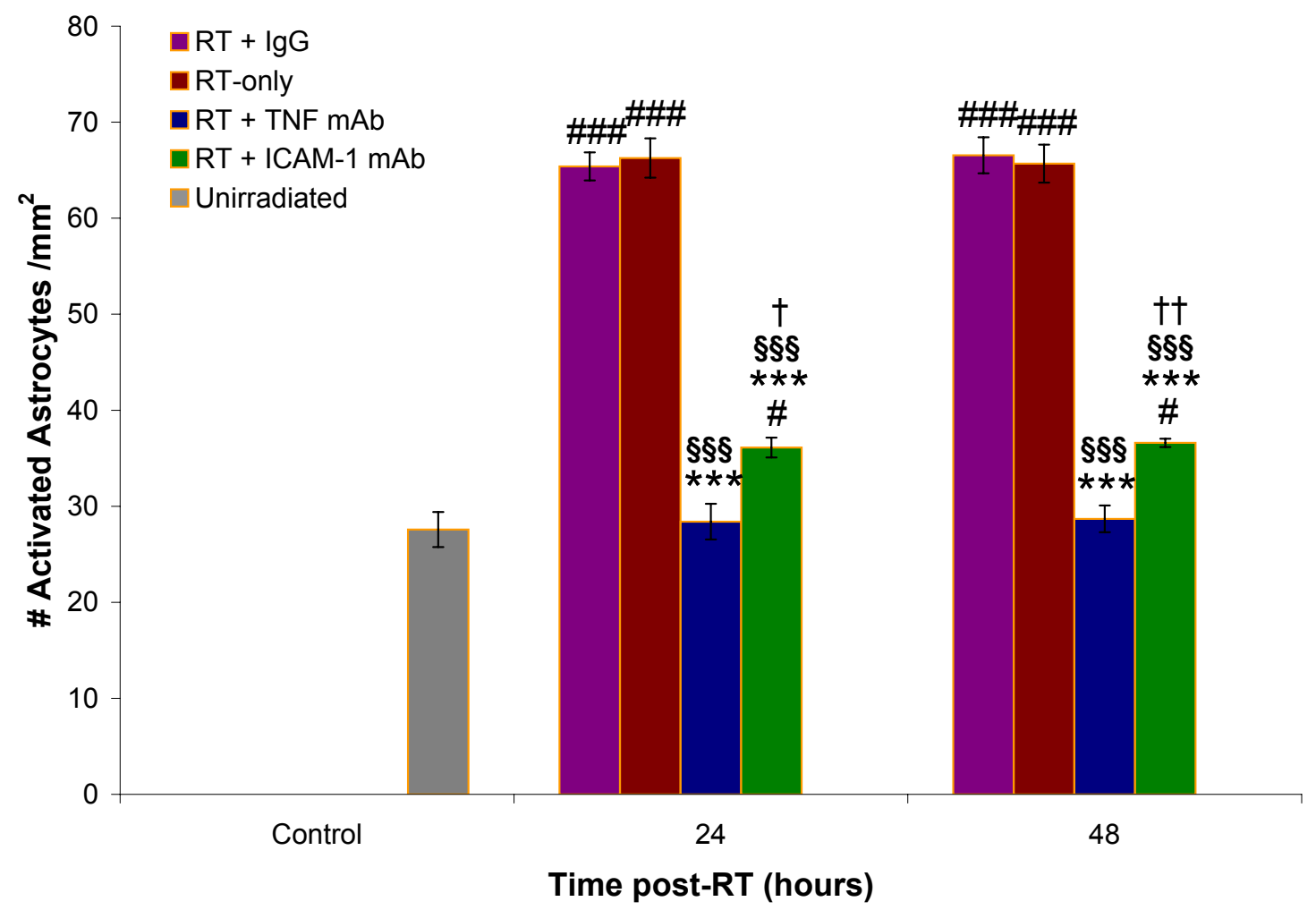

Figure 4.8: Activated astrocyte counts in the dentate gyrus region.

To confirm that the response of the asrocytes following radiation was not limited to the cerebral cortex, counts of astrocyte activation were also made in the dentae gyrus region of the hippocampus. The overall response was consistent with the cerebral cortex, however the overal counts were higher. ${ }^{*}(\mathrm{P}<0.05)$ and ${ }^{* * *}(\mathrm{P}<0.001)$ indicate a significant difference versus control. \#\#\# $(P<0.001)$ indicates a significant difference between the radiation and anti-TNF or radiation and anti-ICAM-1 treated group and the RT-only group at that time point. $\dagger(P<0.05)$ and $\dagger \dagger(P<0.01)$ indicate a significant difference between the radiation and anti-TNF treated group and the radiation and anti-ICAM-1 treated group at that timepoint. $\S \S \S(P<0.001)$ indicates a significant difference versus radiation and IgG treatment at that time point. 


\subsection{Immunofluorescence}

Immunofluorescence showed an increase in protein expression of TNF and ICAM-1 at 24-and 48-hrs post-radiation in the radiation-only group, Figure 4.9. There is specific staining of TNF and ICAM-1 localized around vessels structures, while there is no staining observed in the unirradiated brain. There is no expression of TNF and minimal expression of ICAM-1 observed in the animals irradiated and treated with anti-TNF. In animals treated with radiation and anti-ICAM-1 there is no expression of ICAM-1 and minimal expression of TNF. Observations were made in images at the same imaging settings.

\subsection{Effect of TNF on cerebral vasculature}

When recombinant TNF (rTNF) was administered to evaluate the role of TNF in radiation-induced adhesion and arteriole diameter changes, a significant decrease in arteriole diameter was observed. At control, the average arteriole diameter was $35.28 \pm$ $2.12 \mu \mathrm{m}$, and at 7-hrs post-rTNF injection, the average arteriole diameter had significantly decreased to $30.27 \pm 2.55 \mu \mathrm{m}(\mathrm{P}<0.05)$, Figure 4.10. By 24-hrs post-rTNF injection, the arteriole diameter was $31.34 \pm 2.94 \mu \mathrm{m}(\mathrm{P}>0.05)$.

The administration of rTNF also caused a significant increase in leukocyte adhesion in the venules and arterioles at 7- and 24-hrs post-injection, Figure 4.11. In the arterioles there was a 92\% increase at 7-hrs $(P<0.001)$ and a 33\% increase $(P<0.01)$ at 24-hrs postinjection. In the venules there was a $750 \%$ increase $(P<0.01)$ at 7 -hrs and a $148 \%$ increase $(\mathrm{P}<0.01)$ at 24-hrs post-injection.

When rTNF was administered and immunohistochemical staining for GFAP was performed, a significant amount of activated astrocytes were observed compared to the unirradiated, Figure 4.12. The activated astrocyte count at 7-hrs was $23.42 \pm 0.21$ activated astrocytes $/ \mathrm{mm}^{2}$, which is significantly different than the unirradiated. At 24-hrs the activated astrocyte count was $26.57 \pm 0.29$ activated astrocytes $/ \mathrm{mm}^{2}$, which is significantly higher than the unirradiated and not significantly different than the radiation-only group

\subsection{Discussion}

The goal of this work was to characterize the acute effects of radiation on the brain in a mouse model, and to identify a mechanism of damage. The specific questions that were investigated are:

- What is the role of TNF and ICAM-1 in the acute changes observed following radiation?

Radiation was shown to induce changes in permeability of the BBB, leukocyte adhesion in pial venules, and arteriole diameter at 24- and 48-hrs following localized cranial irradiation. It was shown that when TNF or ICAM-1 were inhibited, via specific antibodies, these vascular changes were inhibited. In addition, radiation-induced 
Figure 4.9: Immunofluorescence staining.

Representative images of the protein expression of TNF and ICAM-1 at 24-hrs post-RT. $(A-B)$ unirradiated, (C-D) irradiated only, (E-F) radiation and anti-TNF treatment, $(\mathrm{G}-\mathrm{H})$ radiation and anti-ICAM-1 treatment. (I-J) Show TNF and ICAM-1 protein expression following the administration of $0.5 \mu \mathrm{g}$ recombinant TNF. Magnification 400x. Images were taken by first focusing on a DAPI image to ensure that the field was in focus. Each image presented is representative of $\mathrm{n}=3$ animals. Scale bar $=50 \mu \mathrm{m}$. 
TNF
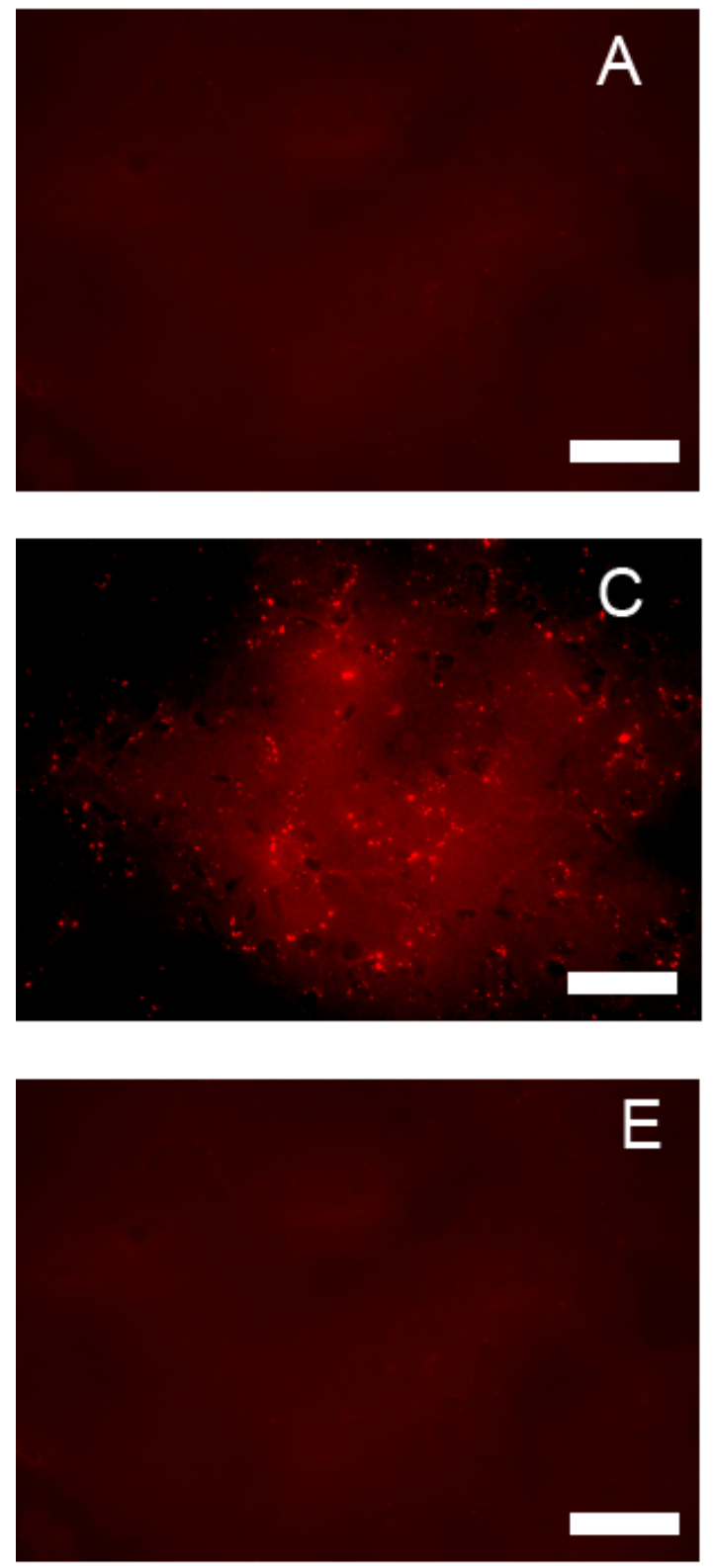

ICAM-1

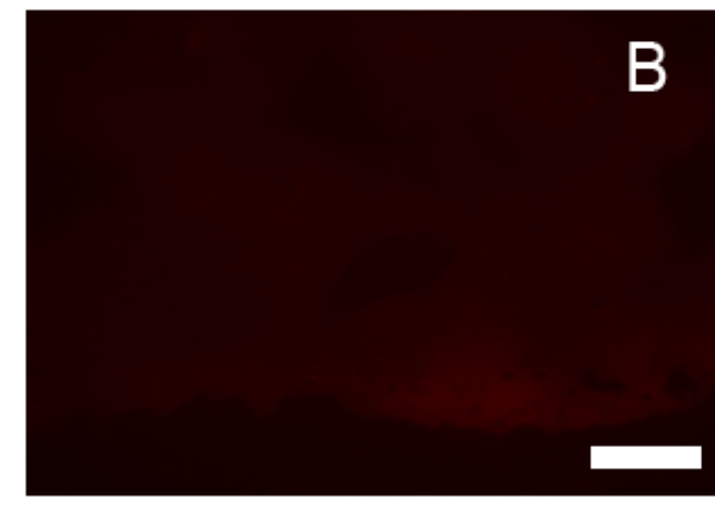

D

F 


\section{TNF}
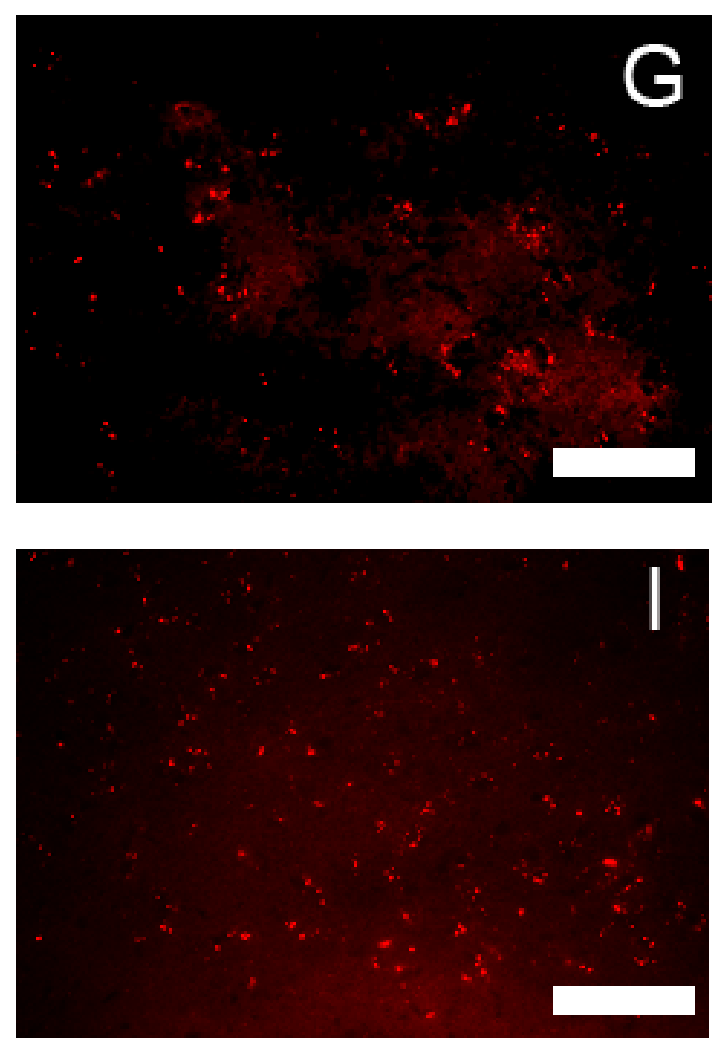

Figure 4.9: (continued). 


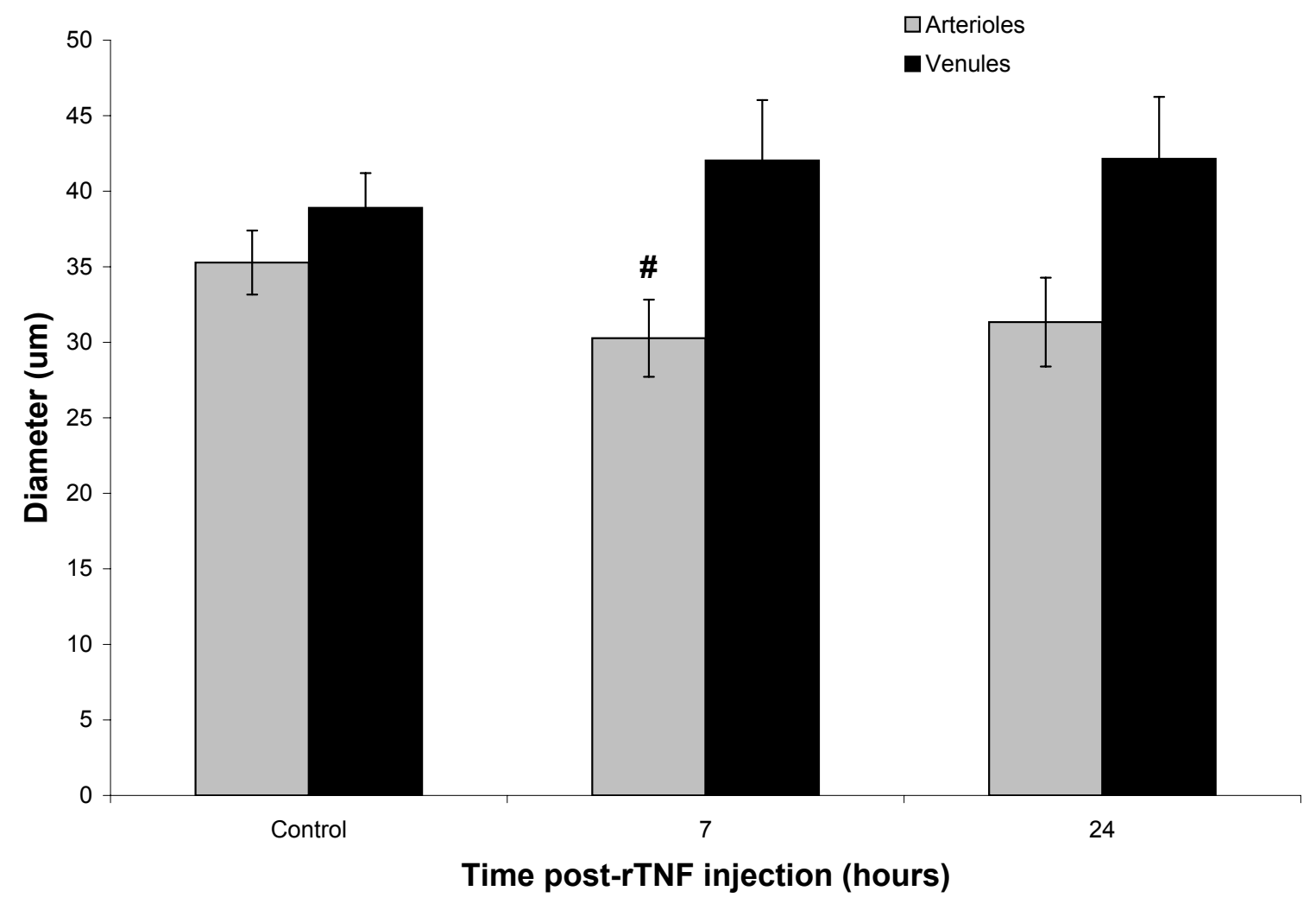

Figure 4.10: TNF induces changes in vessel diameter.

When rTNF is administered there is an observable decrease in arteriole diameter at 7-hrs post-injection. This indicates that the TNF pathway may be the reason for the RT-induced vessel diameter changes, as no changes are seen when the pathway is inhibited. 


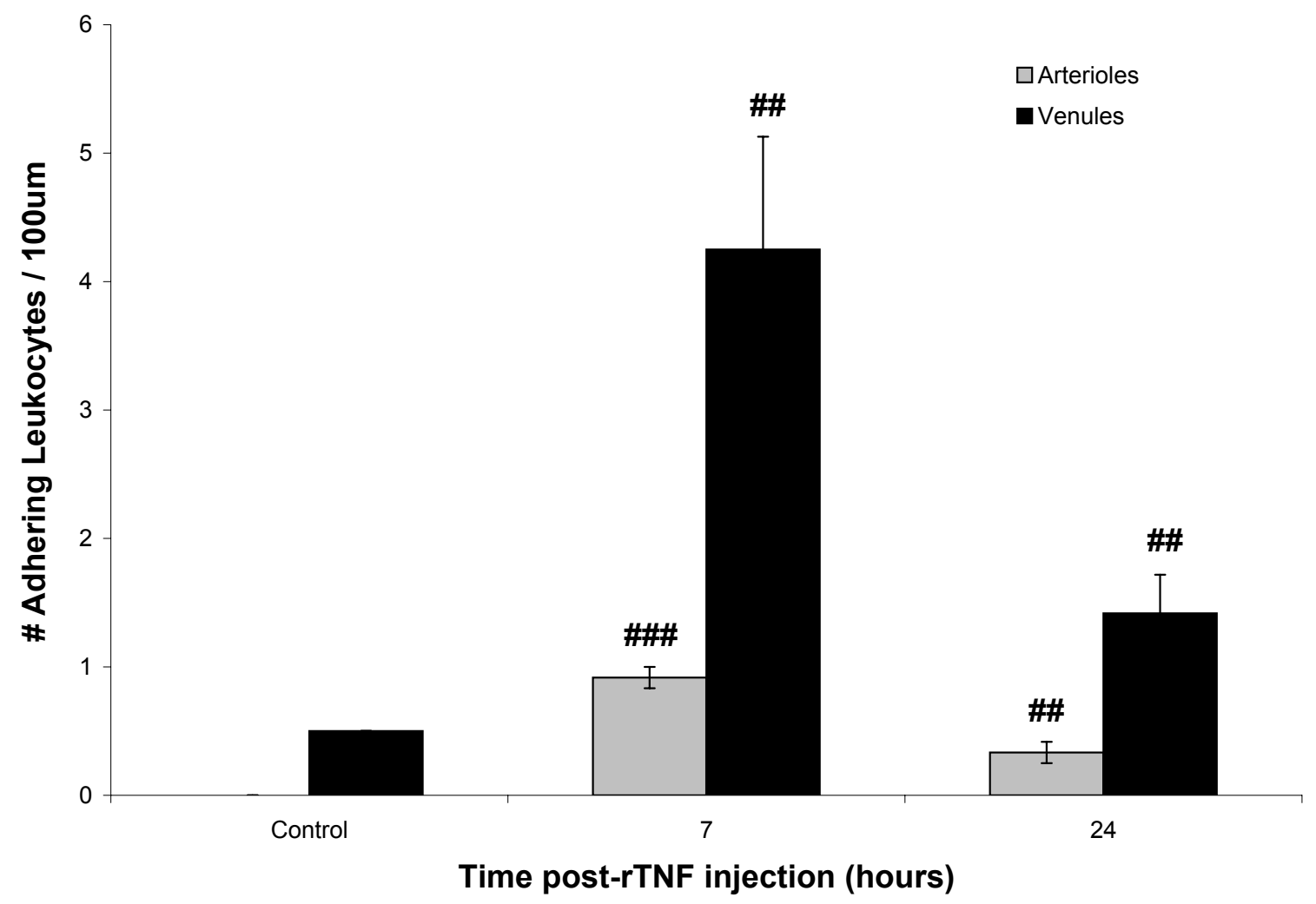

Figure 4.11: TNF induces leukocyte adhesion.

When rTNF is administered there is an observable increase in leukocyte adhesion in both the arterioles and venules at 7- and 24-hrs post-injection of recombinant TNF. This indicates that the TNF pathway is at least partly responsible for the RT-induced changes in leukocyte adhesion, as rTNF induces leukocyte adhesion and no change in leukocyte adhesion is observed when the pathway is inhibited. 


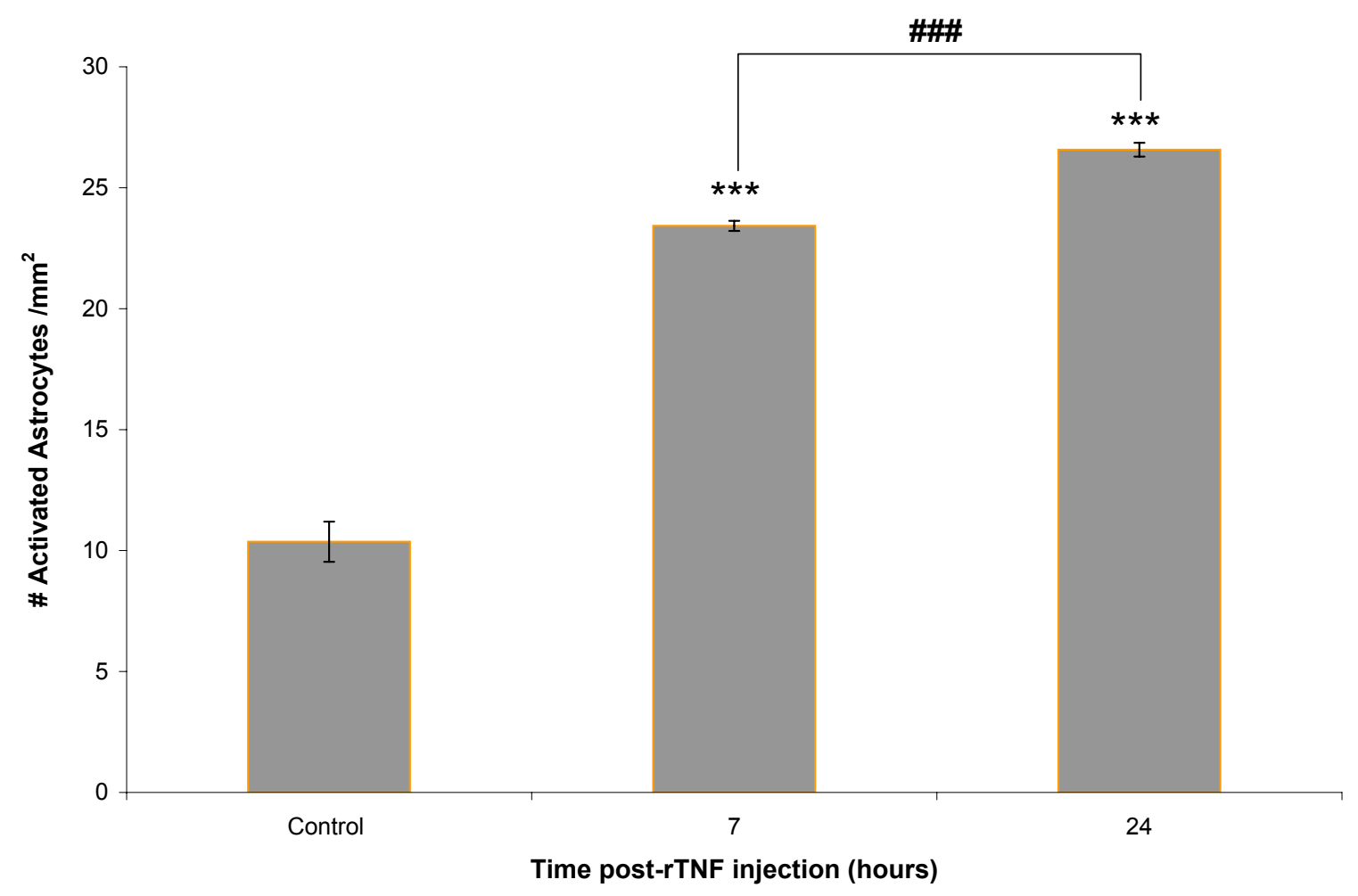

Figure 4.12: TNF induces astrogliosis.

The administration of recombinant TNF induced a significant increase in activated astrocytes at 7- and 24-hrs post-injection. There is also a significant difference between the number of activated astrocytes between 7-hrs post-injection and 24-hrs post-injection. At 24-hrs post-rTNF injection, the number of activated astrocytes is not significantly different than RT-only, see Figure 4.6. 
astrocyte activation was evaluated, and it was shown that the inhibition of TNF prevented astrocyte activation, while the inhibition of ICAM-1 only significantly reduced the activation. This shows that the inflammatory molecules TNF and ICAM-1 are involved in the acute radiation-induced vascular changes in the brain.

- Does inhibiting TNF affect the levels of ICAM-1 and vise versa?

Immunofluorescece staining showed that radiation induces the up-regulation on TNF and ICAM-1 protein expression at 24- and 48-hrs post-irradiation. When antibodies to TNF were administered in conjunction with radiation, the protein expression of both TNF and ICAM-1 were inhibited. When antibodies to ICAM-1 were administered in conjunction with radiation, the protein expression of ICAM-1 was inhibited, but the protein expression of TNF was similar to the irradiated-only animal. This shows that the antibodies used were specific to their respective molecules, and that the inhibition of TNF is more potent than he inhibition of ICAM-1.

- Is there a correlation between the increase in vascular permeability and leukocyte adhesion? Does blocking either molecule, TNF or ICAM-1, disrupt this correlation?

Using a linear regression model, it was shown that there is a strong correlation between the increase in permeability of the BBB and the increase in leukocyte adhesion following irradiation. This is the first time that the correlation between our two main in vivo measurements has been determined. 


\section{Chapter 5}

\section{KZ-41: An Interventional Therapy}

$\mathrm{KZ}-41$ is a potent analog of quinic acid, which is an active ingredient in hot water extracts of the herbal cat's claw (uncaria tomentosa). Studies have shown that the antiinflammatory activity of cat's claw extracts is through the inhibition of NF- $\kappa \mathrm{B}$, and that this inhibition is coming from the quinic acid carboxyl alkyl ester found in the extract [118]. Aqueous extracts from the bark or root bark are used therapeutically in a diversity of diseases including allergy, arthritis, chemotherapy side effects, cancer, bacterial/fungal infections, gastrointestinal inflammation and gastric ulcers [119, 120, 121, 122, 123]. Oral administration of C-Med-100® to humans at doses of $250-750 \mathrm{mg}$ daily has been shown to enhance immune cell response and DNA repair [124, 125, 126]. In addition, preliminary studies from our group indicate that KZ-41 has potent vascular anti-inflammatory effects, promotes vascular recovery from injury, and increases white blood cell count, making it an excellent candidate as a potential agent that facilitates tissue recovery and wound repair.

In this work, the anti-inflammatory effects of KZ-41 are explored as a possible interventional therapy following cranial radiation.

Hypothesis: KZ-41, a derivative of quinic acid that shows anti-inflammatory effects through the NF- $\kappa \mathrm{B} / \mathrm{ICAM}-1$ pathway, can be used to inhibit the inflammation-mediated acute radiation effects.

Specific Aim: Evaluate the effectiveness of $\mathrm{KZ}-41$ as an interventional therapy for acute radiation damage to the blood-brain barrier following a single dose of 20-Gy localized cranial irradiation and compare to the response observed from the direct inhibition of the inflammatory molecules TNF and ICAM-1 via antibodies.

The specific questions being investigated are:

- Is KZ-41 able to inhibit the radiation-induced vascular changes known to be effected by TNF and ICAM-1?

- Does KZ-41 affect the levels of TNF or ICAM-1 following localized cranial radiation?

- If KZ-41 works through the ICAM-1 pathway only, does it decouple the vascular response from the astrocytic response? 


\subsection{Effect of KZ-41 on radiation-induced changes to the microvasculature}

It has been shown in the previous section that there are significant changes in permeability of the BBB to 4.4-kDa FITC-dextran, leukocyte adhesion, and arteriole diameter changes at 24- and 48-hrs after radiation. It was also shown that directly inhibiting the inflammatory molecules TNF or ICAM-1 would inhibit these changes. KZ-41 is thought to work through the NF- $\kappa \mathrm{B} / \mathrm{ICAM}-1$ pathway, and as such should have similar effects as inhibiting these two molecules.

KZ-41 inhibits radiation-induced increases in the permeability of the BBB. Changes in BBB permeability were measured before and at 24- and 48-hrs post-radiation Prior to radiation, BBB permeability was $13.14 \pm 1.99 \times 10^{-7} \mathrm{~cm} / \mathrm{s}$. At 24- and 48-hrs post-radiation permeability significantly increased to $20.30 \pm 2.36 \times 10^{-7} \mathrm{~cm} / \mathrm{s}(P<0.05)$ and $21.53 \pm$ $3.61 \times 10^{-7} \mathrm{~cm} / \mathrm{s}(\mathrm{P}<0.05)$, respectively. When treated with radiation and $\mathrm{KZ}-41, \mathrm{BBB}$ permeability was significantly lower compared to radiation-only. At 24- and 48-hrs postradiation the respective values were $14.56 \pm 0.36 \times 10^{-7} \mathrm{~cm} / \mathrm{s}(\mathrm{P}<0.05)$ and $14.67 \pm 0.66$ $\times 10^{-7} \mathrm{~cm} / \mathrm{s}(\mathrm{P}<0.05)$. (Figure 5.1).

Leukocyte adhesion in pial venules was significantly lower in animals treated with radiation and KZ-41 versus radiation alone. Prior to radiation, leukocyte adhesion was 0.45 \pm 0.13 adhering leukocytes $/ 100 \mu \mathrm{m}$ and was significantly increased compared to control at 24- and 48-hrs post-radiation: $1.01 \pm 0.25(\mathrm{P}<0.05)$ and $1.02 \pm 0.15(\mathrm{P}<0.01)$, adhering leukocytes $/ 100 \mu \mathrm{m}$, respectively. In animals treated with radiation and $\mathrm{KZ}-41$, there was significantly less adhering leukocytes at $24-(0.5 \pm 0.05$ adhering leukocytes $/ 100 \mu \mathrm{m}$; $\mathrm{P}<0.05)$ and $48-(0.55 \pm 0.15$ adhering leukocytes $/ 100 \mu \mathrm{m} ; \mathrm{P}<0.05) \mathrm{hrs}$ post-radiation compared to radiation-only. There was no adhesion observed in the arterioles. (Figures 5.2 and 5.3).

The presence of inflammation appears to create a period of vasoconstriction in the arterioles following radiation. Treatment with KZ-41 inhibited the radiation-induced inflammatory response effectively enough to inhibit this vasoconstriction. In the radiation-only group, there was a significant decrease in arteriole diameter at 48-hrs post-radiation (22.45 $\pm 1.16 \mu \mathrm{m})$, compared to before radiation $(27.16 \pm 2.17 \mu \mathrm{m})$, Figure 5.4. When treated with radiation and $\mathrm{KZ}-41$ there was no decrease in arteriole diameter observed, making the average arteriole diameter significantly larger at 48-hrs $(30.33 \pm 1.16 \mu \mathrm{m} ; \mathrm{P}<0.01)$ postradiation in the radiation and $\mathrm{KZ}-41$ treated group than in the radiation-only group (22.45 \pm $1.16 \mu \mathrm{m})$. There was no significant change in the diameter of the venules observed.

\subsection{Effect of KZ-41 on the radiation-induced astrocytic response}

Activated astrocytes are known to be a sign of injury in the brain. In addition astrocytes are known to secrete the inflammatory molecules TNF and ICAM-1. Therefore the relationship between the inflammation affecting the vasculature and the astrocytic response is of great interest. In the previous section it was shown that radiation induces the activation of astrocytes and that be inhibiting TNF or ICAM-1 astrocytic acivation can be significantly lowered or inhibited. In the unirradiated brain there were $11.41 \pm 1.21$ activated astrocytes $/ \mathrm{mm}^{2}$, Figures 5.5 and 5.6. At 24- and 48-hrs post-radiation activated astrocyte 


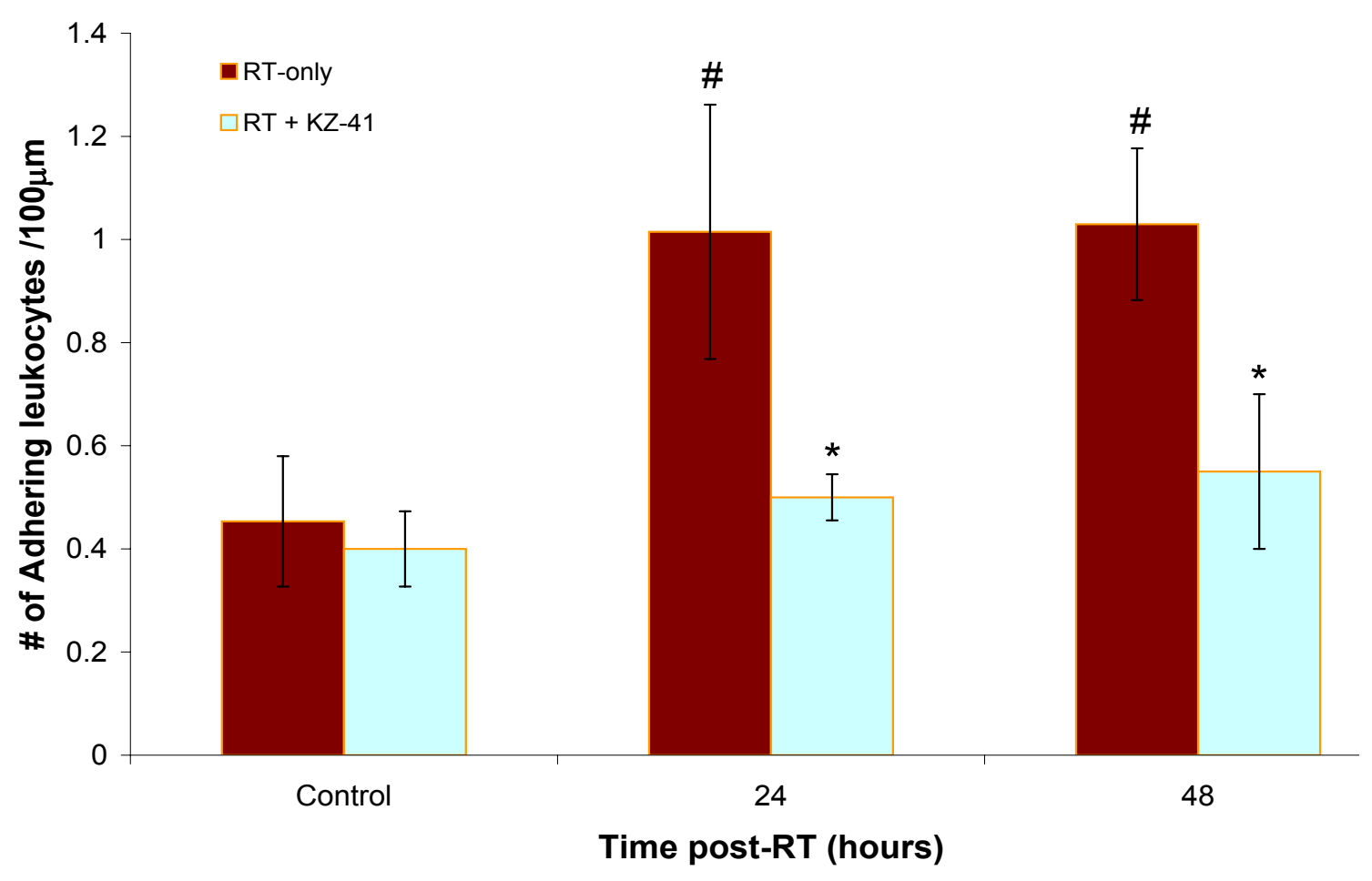

Figure 5.1: Vascular permeability of the BBB following radiotherapy following KZ-41. Radiation induces an increase in vascular permeability of the BBB at 24- and 48-hrs following 20-Gy localized cranial irradiation. When the inflammatory response is limited via treatment with $\mathrm{KZ}-41$, the increase in vascular permeability is inhibited. 


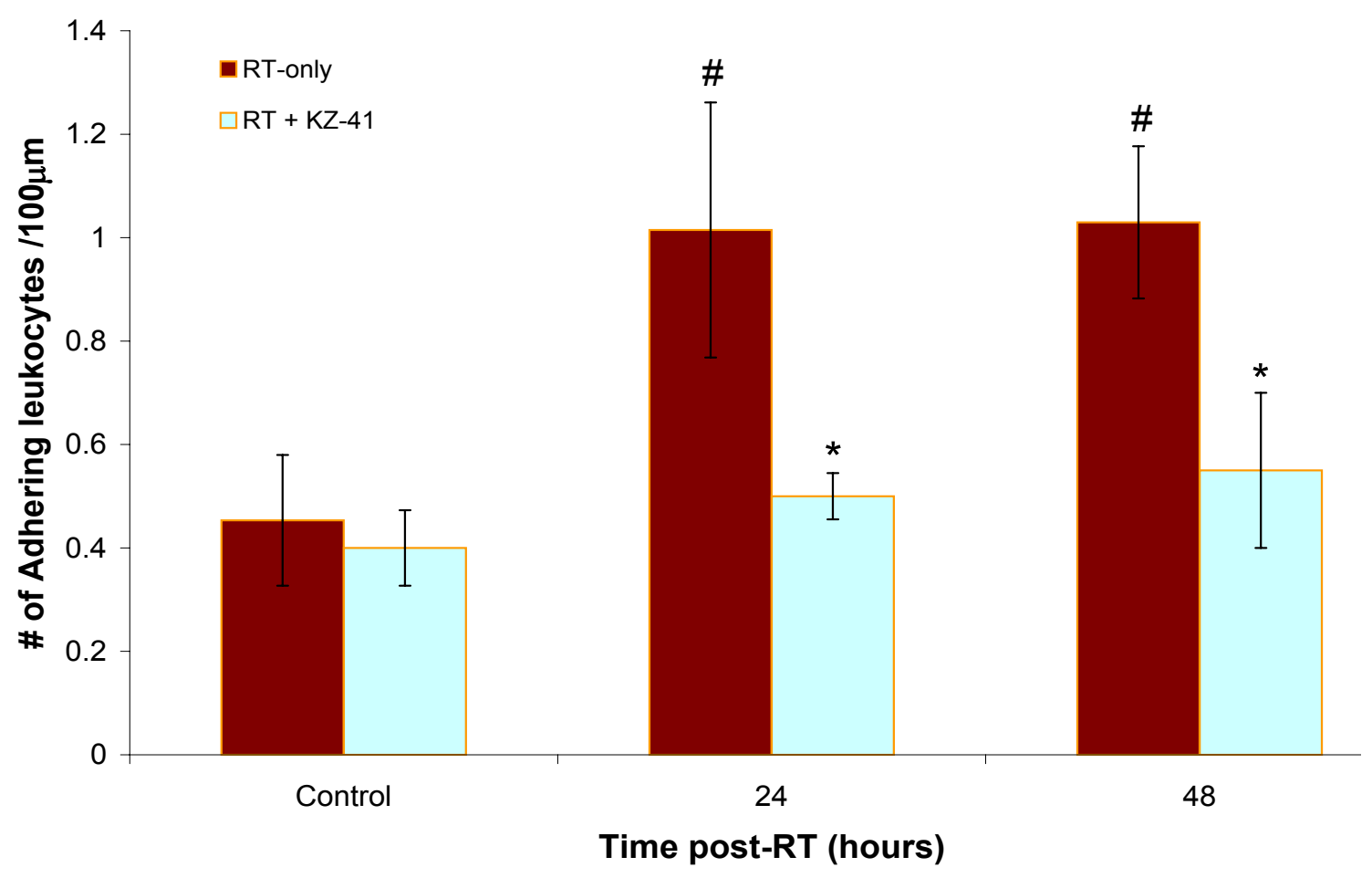

Figure 5.2: Leukocyte adhesion following radiotherapy can be inhibited by KZ-41.

Radiation induces an increase in adhering leukocytes in the pial vessels of the brain at 24and 48-hrs following 20-Gy localized cranial irradiation. When the inflammatory response is limited via treatment with an antibody (anti-TNF or anti-ICAM) or with KZ-41, the increase in adhering leukocytes is inhibited. 

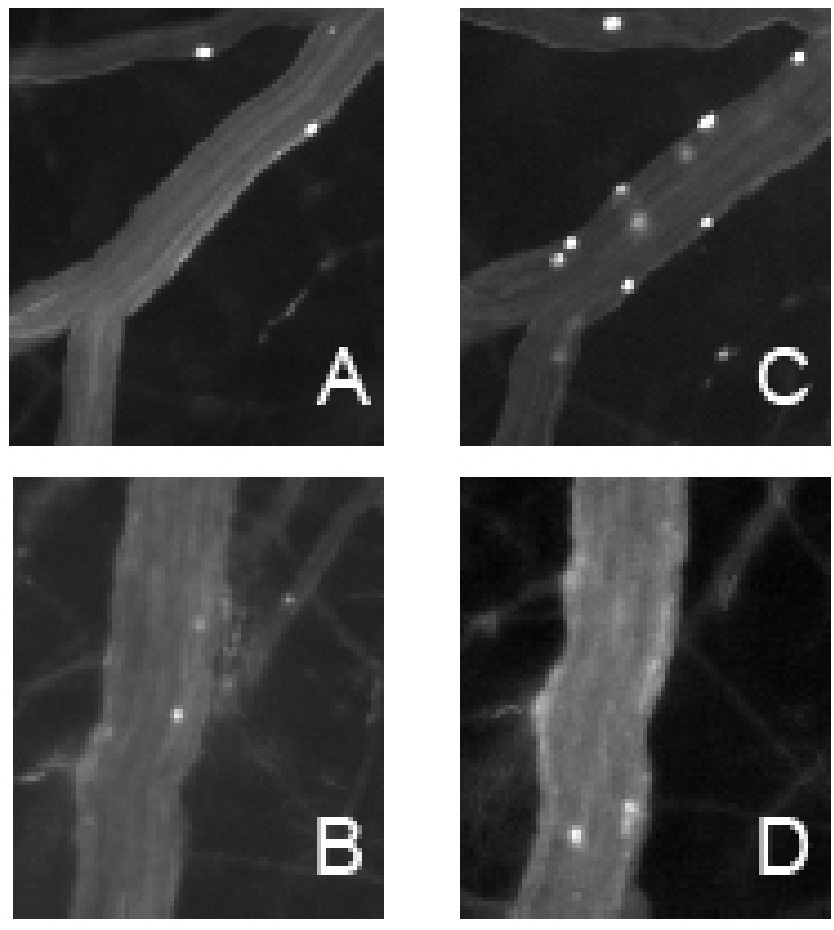

Figure 5.3: Representative images of radiation-induced leukocyte adhesion.

Representative images of the changes observed in leukocyte adhesion 24-hrs following RT. (A-D) Control images with few adherent leukocytes observed (E) 24hrs post-20Gy RTonly; a significant amount of adhering leukocytes are visible. (F-H) 24hrs post-20Gy RT+ anti-TNF, RT + ICAM-1, and RT + KZ-41; when treated prior to radiation, the adhering of leukocytes can be inhibited. Images were taken at approximately the same time after injection of Rhodamine-6G. 


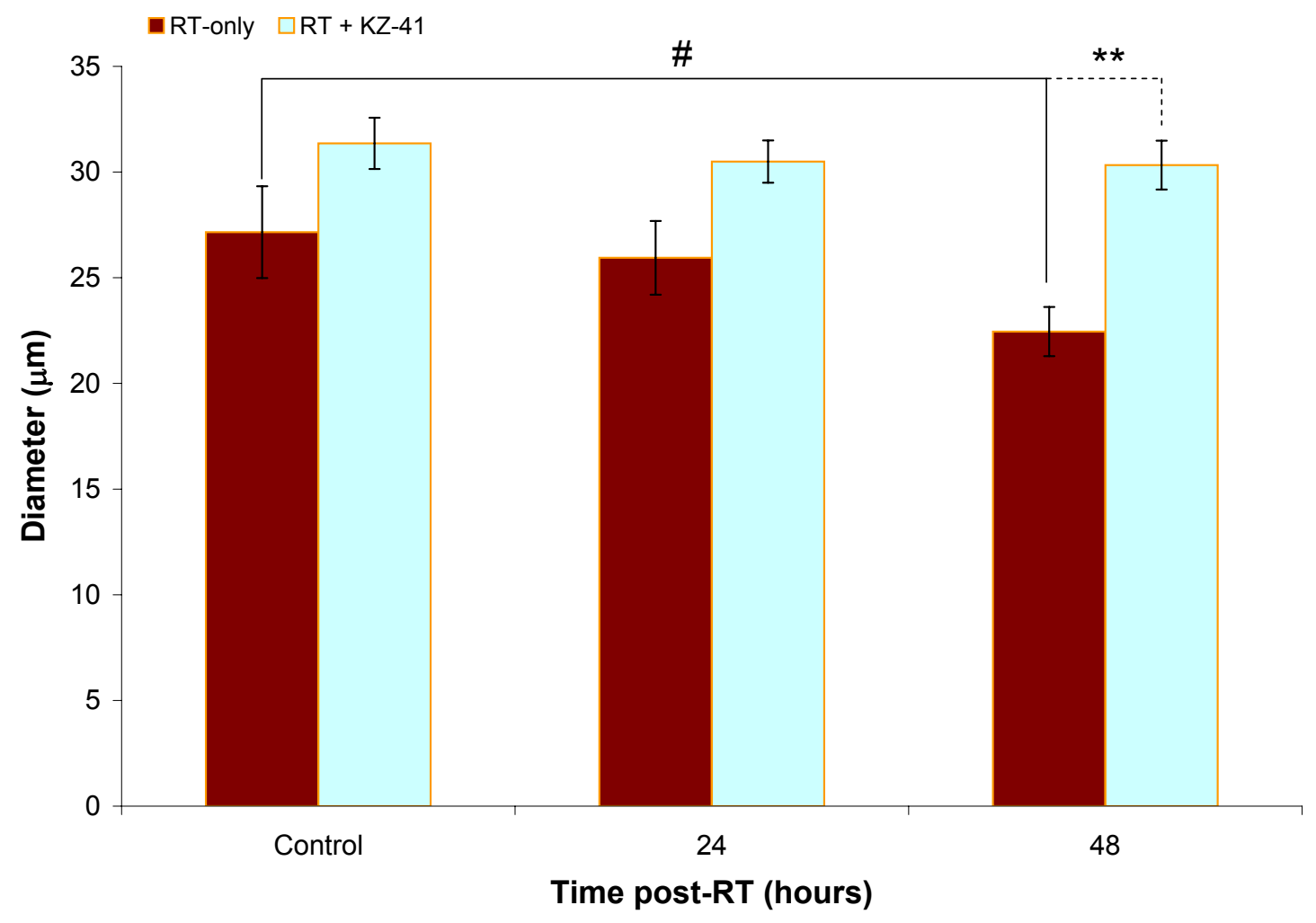

Figure 5.4: Radiation-induced changes in vessel diameter.

Radiation induced a decrease in arteriole vessel diameter at 48-hrs following a single dose of 20-Gy localized cranial radiation. This decrease in vessel diameter is inhibited when the inflammatory response is limited via treatment with KZ-41. 


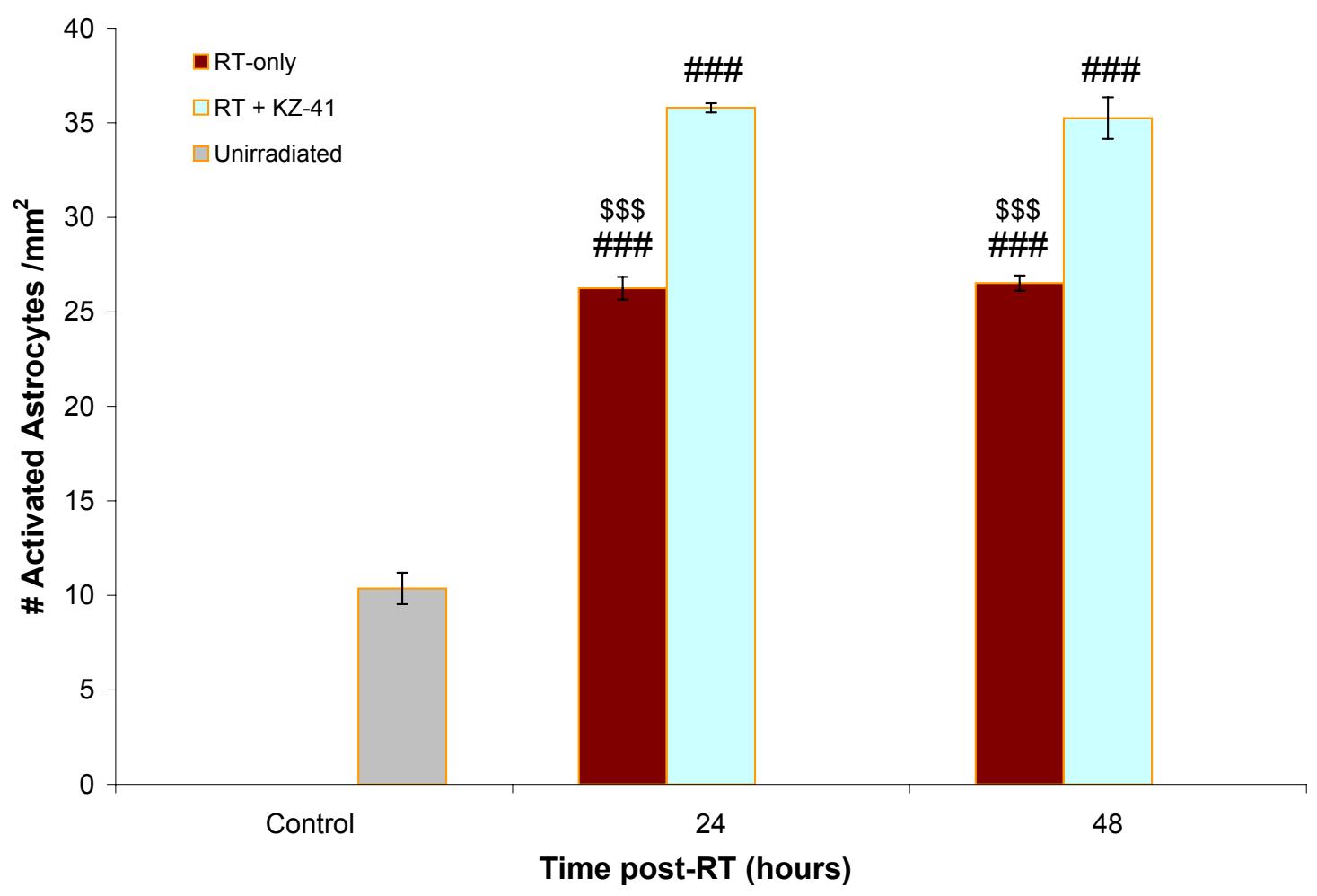

Figure 5.5: Activated astrocyte counts in the cerebral cortex is exacerbated with KZ41.

Following radiation, a significant amount of astrogliosis was observed at 24- and 48-hrs and was significantly inhibited when treated with anti-TNF or anti-ICAM-1. However, KZ-41 induced astrogliosis to levels significantly higher than RT-only. \#\#\# $(P<0.001)$ indicates a significant difference versus control. $\$ \$ \$(P<0.001)$ indicates a significant difference versus radiation and $\mathrm{KZ}-41$ treatment at that time point. 

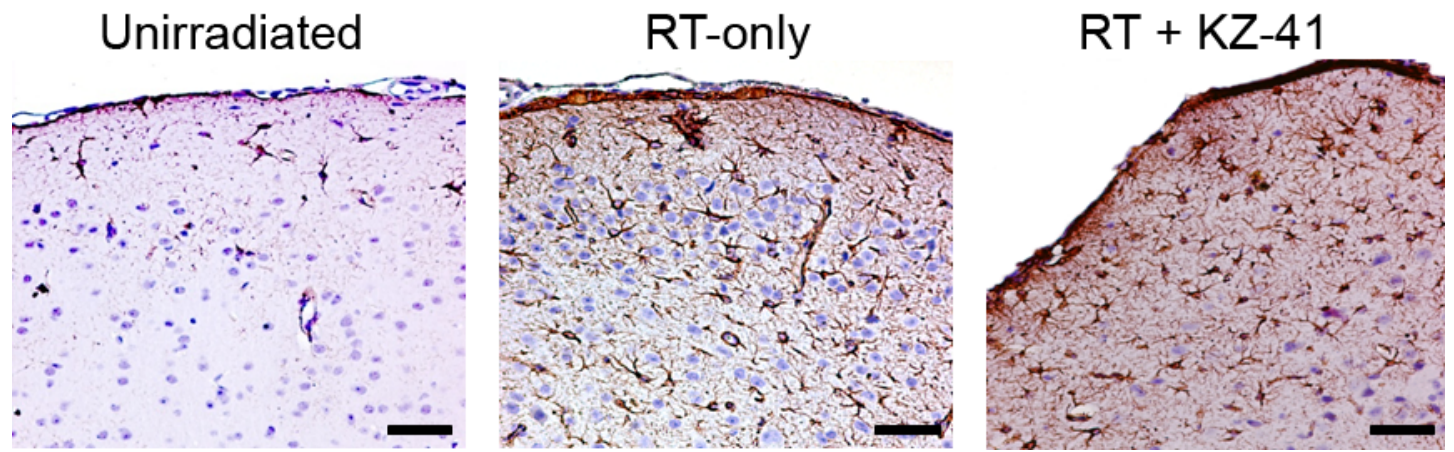

Figure 5.6: GFAP staining for activated astrocytes.

Representative images of GFAP staining at 24-hrs post-radiation with and without treatment with KZ-41. There is a significant difference in activated astrocytes in the radiation and KZ-41 treated group versus the unirradiated and a significant difference between RT-only and radiation and KZ-41. Images were taken at the same exposure settings and scaled the same. Magnification 200x. Each image presented is representative of $\mathrm{n}=3$ animals. Scale bar $=100 \mu \mathrm{m}$. 
counts were $21.92 \pm 0.24(\mathrm{P}<0.001)$ and $22.92 \pm 0.36(\mathrm{P}<0.001)$ activated astrocytes $/ \mathrm{mm}^{2}$, respectively. In animals treated with radiation and $\mathrm{KZ}-41$, the number of activated astrocytes was significantly greater than both the unirradiated group and the radiation-only group. At 24- and 48-hrs post-radiation, the activated astrocytes counts were $33.69 \pm 0.55$ activated astrocytes $/ \mathrm{mm}^{2}$ and $34.38 \pm 0.41$ activated astrocytes $/ \mathrm{mm}^{2}$, respectively.

To confirm that the observed increase of activated astrocytes in the cerebral cortex is consistent in other regions of the brain, activated astrocytes were counted in the dentate gyrus region of the hippocampus. In the unirradiated and radiation-only groups, the astrocytic response in the dentate gyrus region mimicked the response in the cerebral cortex. In the radiation and KZ-41 treated group, however, the significant increase in activated astrocytes over the radiation-only group is lost. There are still significantly more astrocytes in the radiation and KZ-41 group than in the unirradiated brain, but it is no longer significantly different than the radiation-only group, Figure 5.7 .

\subsection{Inhibition of protein expression using KZ-41}

Immunofluorescence staining can be used to visualize protein expression. In the previous section, it was shown that in the unirradiated brain there is virtually no expression of TNF or ICAM-1 at the protein level, while there is expression in the irradiated brain at 24-hrs post-irradiation. When TNF antibody was administered in conjunction with radiation, immunofluorescence staining showed that both TNF and ICAM-1 protein expression was inhibited. As expected, when ICAM-1 antibody was administered in conjunction with radiation, ICAM-1 expression was inhibited, but TNF expression was not different than irradiated-only, Figure 5.8. Similarly in the radiation and KZ-41 treated animals, there is minimal expression of ICAM-1 while TNF expression is most similar to the irradiated-only group. This staining pattern is not unexpected as $\mathrm{KZ}-41$ is thought to work through the NF- $\kappa$ B/ICAM-1 pathway, and not directly inhibit TNF. Observations were made in images at the same imaging settings.

\subsection{Discussion}

The goal of this work was to evaluate the effectiveness of the novel anti-inflammatory agent KZ-41 to limit the inflammatory-mediated radiation response in the brain. The specific questions investigated were:

- Is KZ-41 able to inhibit the radiation-induced vascular changes known to be effected by TNF and ICAM-1?

When KZ-41 was administered in conjunction with radiation, it was able to abrogate the radiation-induced changes to the vasculature. It inhibited: permeability increases in the BBB, leukocyte adhesion in the pial venules, and prevented the vasoconstriction of the arterioles at 48-hrs post-irradiation. 


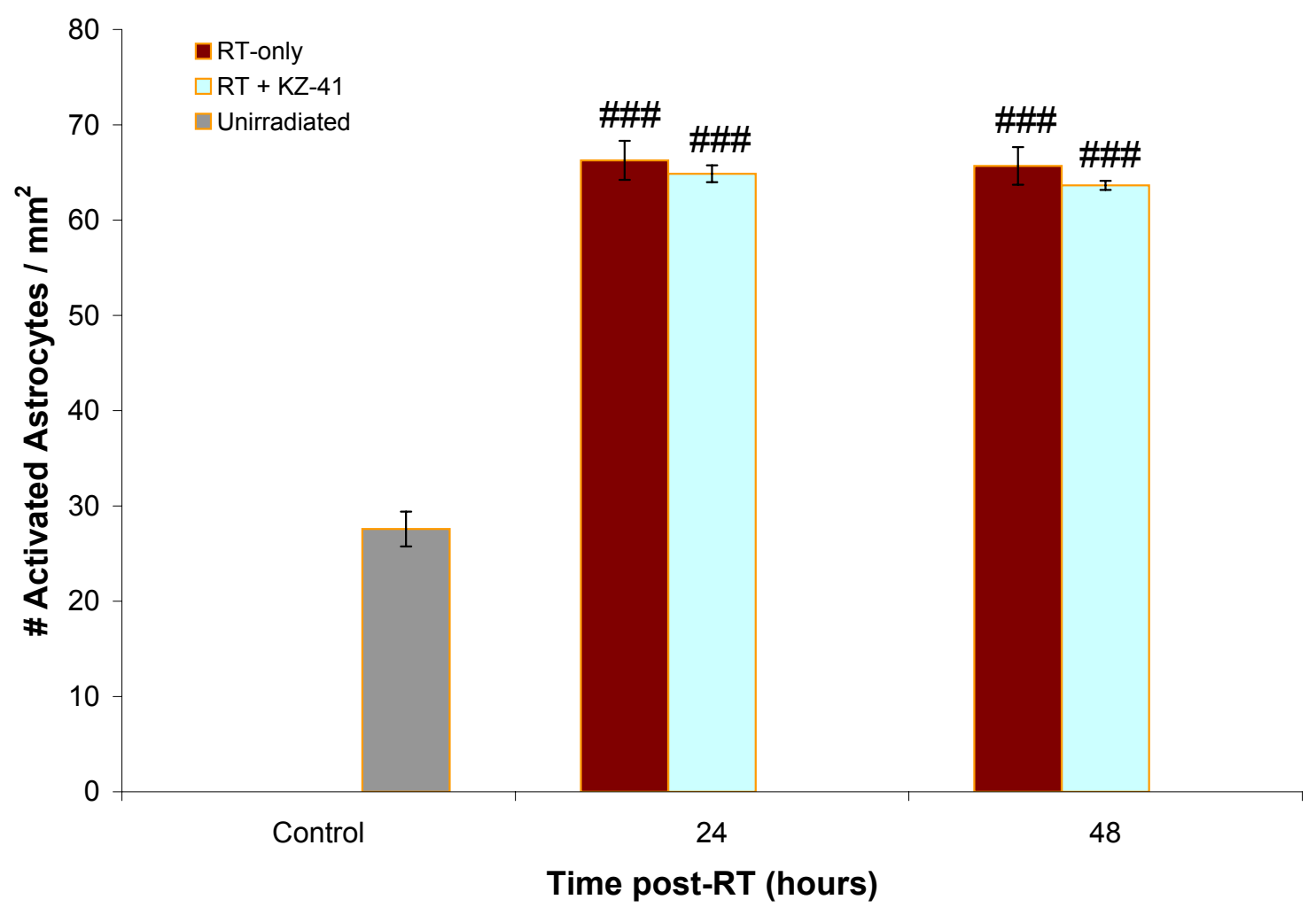

Figure 5.7: Activated astrocyte counts in the dentate gyrus region following radiation and KZ-41.

To confirm that the response of the asrocytes following radiation was not limited to the cerebral cortex, counts of astrocyte activation were also made in the dentate gyrus region of the hippocampus. The overall response was consistent with the cerebral cortex, however the overal counts were higher. However, the significant difference between the number of activated astrocytes in the radiation-only and radiation and KZ-41 treated group was not observed. \#\#\# ( $<<0.001)$ indicates a significant difference versus the unirradiated group. 


\section{TNF}
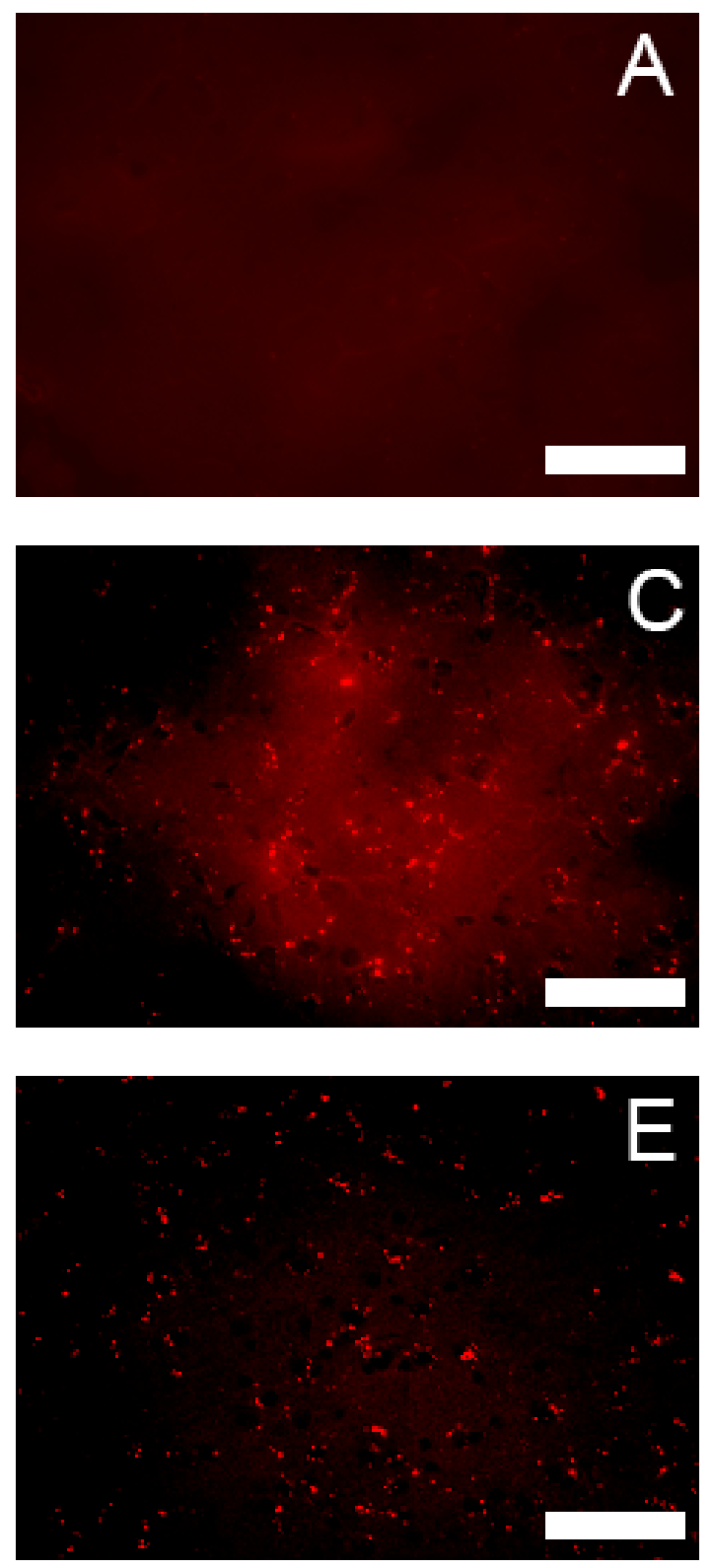

ICAM-1
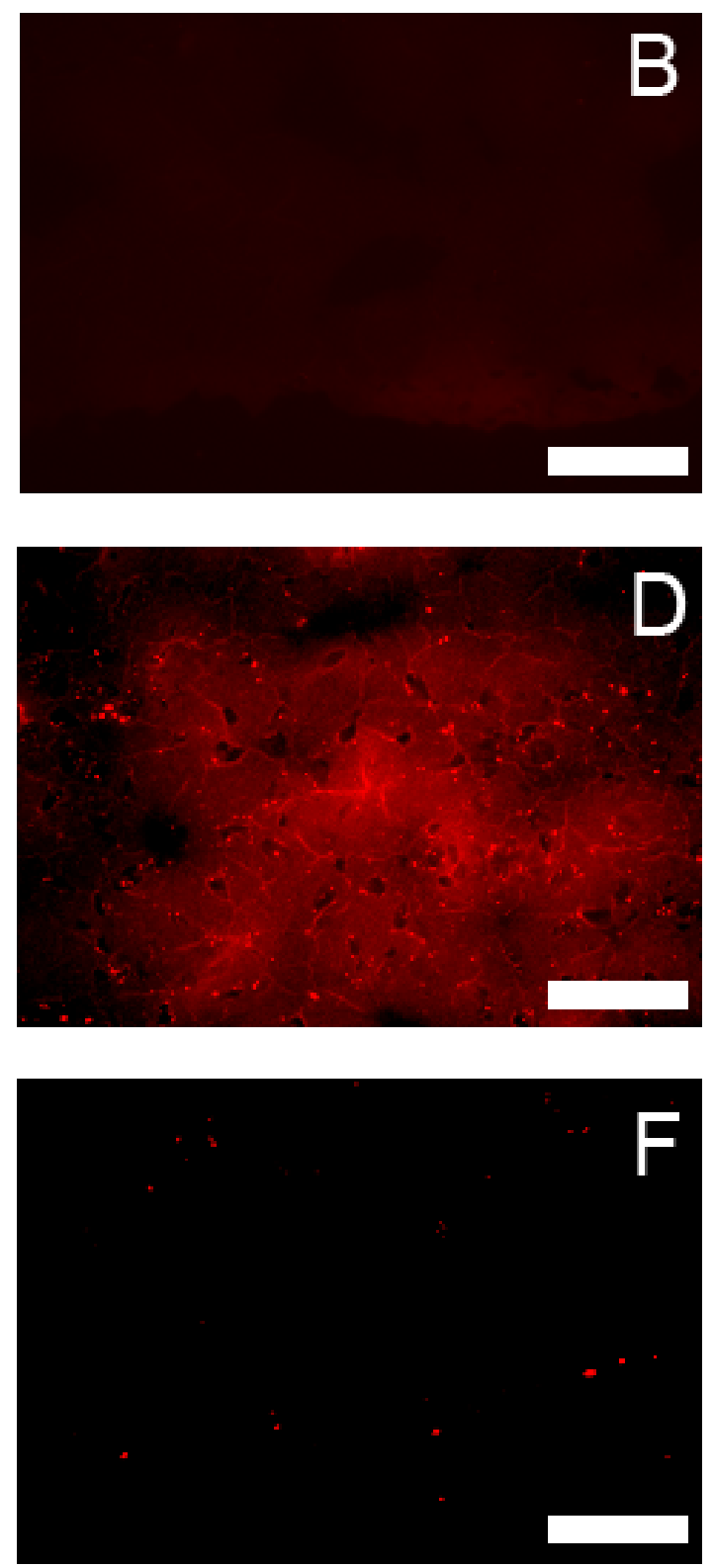

Figure 5.8: Protein inhibition via KZ-41 administration.

Representative images of the protein expression of TNF and ICAM-1 at 24-hrs post-RT. (A-B) unirradiated, (C-D) irradiated only, (E-F) radiation and KZ-41, Z-41. Magnification 400x. Images were taken by first focusing on a DAPI image to ensure that the field was in focus. Each image presented is representative of $n=3$ animals. Scale bar $=50 \mu \mathrm{m}$ 
- Does KZ-41 affect the levels of TNF or ICAM-1 following localized cranial radiation?

It is thought that the anti-inflammatory properties of $K Z-41$, a derivative of quinic acid, are through the inhibition of NF- $\kappa \mathrm{B}$. When KZ-41 was administered in conjunction with cranial irradiation, ICAM-1 protein expression was inhibited while TNF protein expression was not different than irradiation-only. The observed inhibition of ICAM-1 does support the hypothesis that KZ-41 work through the inhibition of NF- $\kappa \mathrm{B}$.

- If KZ-41 works through the ICAM-1 pathway only, does it decouple the vascular response from the astrocytic response?

When KZ-41 was administered in conjunction with radiation, there was an inhibition in the vascular response that was observed at 24- and 48-hrs post-irradiation. However, when activated astrocytes were counted, the inhibition of the vascular changes did not prevent the astrocytic response. In fact, the addition of KZ-41 exacerbaed the astrocyte activation, significantly increasing it compared to radiation alone. This differs from the response seen when specific antibodies were used against the inflammatory molecules TNF and ICAM. When antibodies were administered, both the vascular and astrocytic responses were inhibited. 


\section{Chapter 6}

\section{Early Markers of Radiation-Induced Damage}

Genes are the genetic instructions used in the development and function of all known living organisms. Identifying and understanding genes is the key to understanding how many diseases affect the body, and how to effectively treat these diseases. In October 1990, a 13-year international effort was initiated to sequence the complete human genome. This was known as the Human Genome Project (HGP). With the HGP, 20,000 to 25,000 genes were identified, with approximately 3 million base pairs. Work continues today understanding how genes and gene expression is altered during different disease states.

Figure 6.1 shows a schematic representation of the series of acute response events that occur following localized whole brain radiation. There are three main types of response: protein, serum cytokine, and gene expression. The protein response has been characterized in the previous chapter and includes: increased expression of ICAM-1 and increased cell adhesion and infiltration leading to damage of the microvascular network. Serum cytokines are proteins secreted by various cells, which regulate immunity, inflammation, and hematopoiesis, measured in serum rather than in whole blood. Cytokines act by binding to specific receptors, which then trigger a cell to alter its behavior. The third type of response is from changes in gene expression. This work begins to understand the changes in the gene profile of the irradiated brain in an attempt to better understand radiation damage.

Hypothesis: The gene profile of the acute damage following radiation can give an insight into the inflammatory, vascular, and parenchymal pathways that could be targeted for interventional therapy to abrogate radiation-induced late effects.

Specific Aim: Investigate the acute inflammatory, vascular, parenchymal, and neurostructural changes in the brain following radiation damage by characterizing the gene response using microarray gene expression analysis.

The specific questions being investigated are:

- Is there a paracrine response that occurs?

- Can we distinguish between parenchymal and vascular damage?

- Are there early signs of neurostructural damage? 


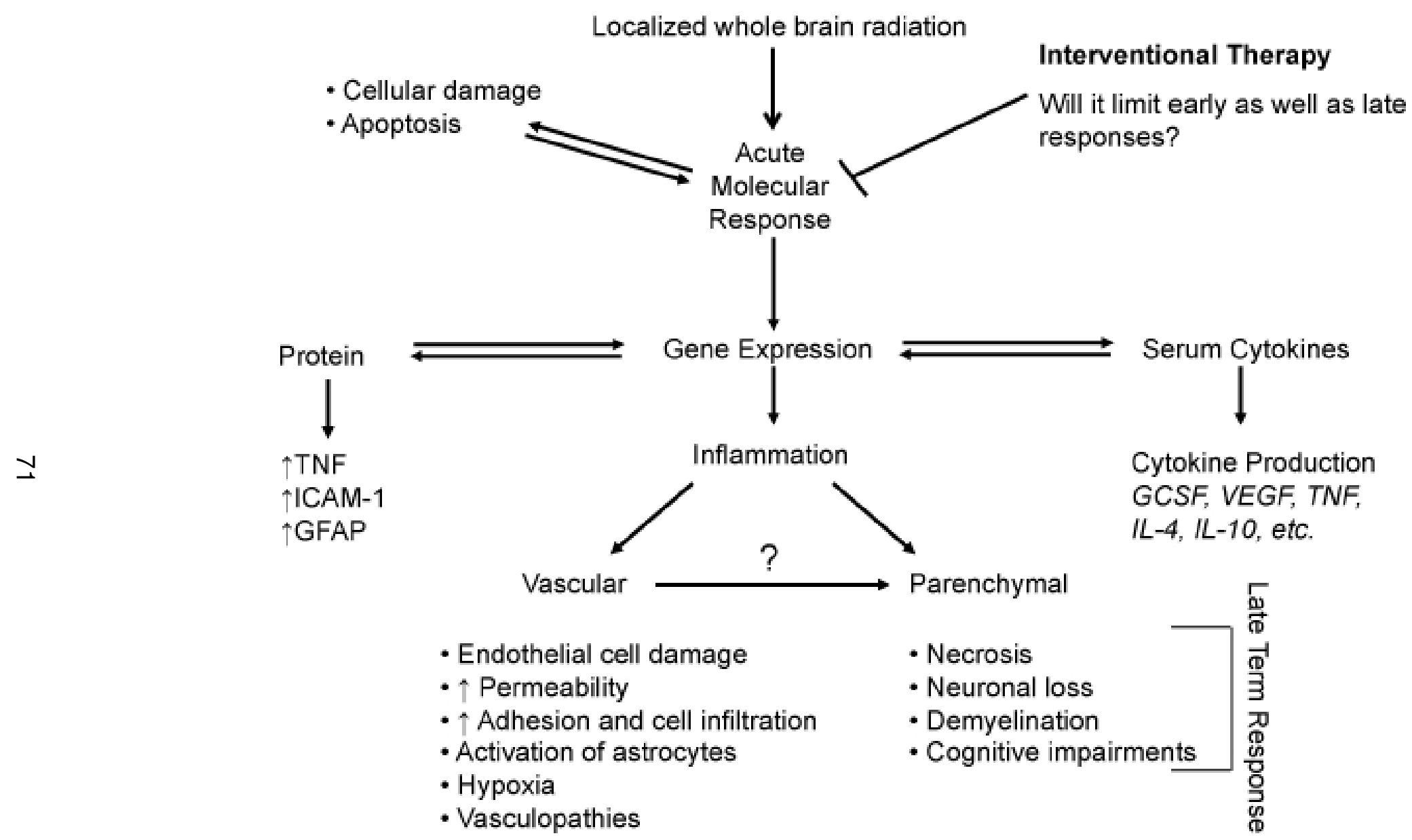

Figure 6.1: Schematic of the acute molecular responses following radiation. 


\subsection{Microarray analysis}

The changes in gene expression following a single dose of 20-Gy radiation localized to the brain, were evaluated to extend our in vivo analysis to the most basic level of change. At 2-hrs following a single dose of 20-Gy localized cranial irradiation there were 2514 genes significantly different versus control at $\mathrm{P}<0.05$, and 656 genes significantly different than control at $\mathrm{P}<0.01$, Table 6.1. Of the 2514 genes significantly different at the $\mathrm{P}<0.05$ level, 64 showed a fold change of 2 or greater $(26$ genes up-regulated and 38 genes downregulated). In addition, 409 genes showed a fold change of 1.5 or greater with 141 genes up-regulated and 268 genes down-regulated. The time point of 2-hrs post-irradiation was chosen because previous work by Sabek et al. showed that radiation-induced changes in TNF and ICAM-1 mRNA peaked at this time [57].

When all of the significant genes (with $\mathrm{P}<0.05$ ) are considered, the most significantly altered pathways are: MAPK signaling, focal adhesion, regulation of actin cytoskeleton, cell cycle, and tight junction. MAPK signaling has been linked to many different cellular events including, proliferation, senescence (a type of cell death), differentiation, and apoptosis. Focal adhesions are altered in response to changes in the molecular composition in a cells extracellular matrix environment, and have been linked to pathways that regulate cell proliferation, survival, migration and invasion. Actin is a component of every cells cytoskeleton, and regulates many cellular functions including muscle contraction, cell motility, division and signaling, cytokinesis, vesicle movement, and the maintenance of cell junctions and cell shape. The cell cycle is a series of events leading to cells replication. It has been shown that the inhibition of the cell cycle is critical for many disease including cancer and inflammation, however a proper cell cycle process is necessary for the recovery of tissue following radiation. The tight junction pathway regulates the production of tight junction proteins, including claudin- 5 and occludin, that are responsible for maintaining the firm adherence of consecutive endothelial cells along the vasculature of the brain, a prominent characteristic of the blood-brain barrier. The disruption of the tight junctions is a known cause for the observed increase in BBB permeability following cranial irradiation.

\subsection{Paracrine response}

A paracrine response is when a substance secreted by one cell acts on adjacent cells. A common example of a paracrine response in the brain is the inflammatory/immune response. Following radiation with a single dose of 20-Gy radiation localized to the brain, there are 36 genes that are significantly modulated (Table 6.2) and involved in the inflammatory/immune response. From these genes, it can be seen that the cytokine-cytokine receptor interaction pathway (Table 6.3) is the most altered, with seven genes being altered. All seven of the genes are classified as cytokines, with six classified as chemotactic cytokines, or chemokines. A main responsibility of chemokines is to guide the migration of response cells to the source of the chemokine. This is done by creating a chemotaxic chemokine gradient. In other words, the chemokines that are released by the damaged cells form a concentration gradient of chemokines, allowing the responding cells to use the gradient to find the source of damage. The chemokines that are modulated are proinflammatory, and are known to be induced during an immune response in order to recruit 
Table 6.1: Significant genes by function/pathway.

\begin{tabular}{ll}
\hline \multirow{2}{*}{ System } & \multicolumn{2}{c}{$\#$ of Genes } \\
\cline { 2 - 3 } & $\mathrm{P}<0.05 \quad \mathrm{P}<0.01$ \\
\hline
\end{tabular}

\section{Genetic Information Processing}

\section{Transcription}

RNA polymerase

Basal transcription factors

30

42

\section{Translation}

Ribosome

Aminoacyl-tRNA biosynthesis

$3 \quad 0$

10

Folding, Sorting and Degradation

Protein export

SNARE interactions in vesicular transport

Regulation of autophagy

Proteasome

\section{Environmental Information Processing}

Membrane Transport

ABC transporters

4

2

Signal Transduction

MAPK signaling pathway

Wnt signaling pathway

2

Notch signaling pathway

Hedgehog signaling pathway

$17 \quad 4$

92

TGF-beta signaling pathway

$3 \quad 1$

VEGF signaling pathway Jak-STAT signaling pathway

93

Calcium signaling pathway

$5 \quad 1$

$14 \quad 5$

Phosphatidylinositol signaling system mTOR signaling pathway

$17 \quad 2$

$6 \quad 2$

$7 \quad 3$

Signaling Molecules and Interaction

Neuroactive ligand-receptor interaction

Cytokine-cytokine receptor interaction

ECM-receptor interaction

Cell adhesion molecules (CAMs)

$\begin{array}{cc}12 & 4 \\ 16 & 7 \\ 3 & 0 \\ 14 & 7\end{array}$


Table 6.1: (continued).

\begin{tabular}{lll}
\hline \multirow{2}{*}{ System } & \multicolumn{2}{c}{ \# of Genes } \\
\cline { 2 - 3 } & $\mathrm{P}<0.05$ & $\mathrm{P}<0.01$ \\
\hline
\end{tabular}

\section{Cellular Processes}

Cell Motility

Regulation of actin cytoskeleton

$21 \quad 2$

Cell Growth and Death

Cell cycle

$19 \quad 4$

Apoptosis

$10 \quad 2$

Cell Communication

Focal adhesion

$21 \quad 6$

Adherens junction

$12 \quad 2$

Tight junction

$17 \quad 5$

Gap junction

120

Endocrine System

Insulin signaling pathway

Adipocytokine signaling pathway

$14 \quad 2$

PPAR signaling pathway

92

$\mathrm{GnRH}$ signaling pathway

$6 \quad 1$

$11 \quad 1$

\section{Immune System}

Hematopoietic cell lineage $\quad 3 \quad 1$

Complement and coagulation cascades $\quad 4 \quad 1$

$\begin{array}{lll}\text { Toll-like receptor signaling pathway } & 12 & 7\end{array}$

Natural killer cell mediated cytotoxicity $10 \quad 3$

Antigen processing and presentation $\quad 9 \quad 4$

T cell receptor signaling pathway $\quad 12 \quad 5$

$\begin{array}{lll}\text { B cell receptor signaling pathway } & 7 & 3\end{array}$

$\begin{array}{lll}\text { Fc epsilon RI signaling pathway } & 7 & 1\end{array}$

Leukocyte transendothelial migration $\quad 9 \quad 3$

Nervous System

Long-term potentiation $\quad 9 \quad 3$

Long-term depression

$9 \quad 1$

Sensory System

Olfactory transduction $\quad 2 \quad 0$

Taste transduction

30


Table 6.1: (continued).

\begin{tabular}{|c|c|c|}
\hline \multirow[b]{2}{*}{ System } & \multicolumn{2}{|c|}{ \# of Genes } \\
\hline & $P<0.05$ & $\mathrm{P}<0.01$ \\
\hline \multicolumn{3}{|l|}{ Development } \\
\hline Dorso-ventral axis formation & 3 & 0 \\
\hline Axon guidance & 16 & 9 \\
\hline \multicolumn{3}{|l|}{ Behavior } \\
\hline Circadian rhythm & 2 & 0 \\
\hline \multicolumn{3}{|l|}{ Diseases } \\
\hline \multicolumn{3}{|l|}{ Cancers } \\
\hline Colorectal cancer & 15 & 4 \\
\hline Pancreatic cancer & 8 & 1 \\
\hline Glioma & 15 & 3 \\
\hline Chronic myeloid leukemia & 14 & 3 \\
\hline \multicolumn{3}{|l|}{ Neurodegenerative Diseases } \\
\hline Alzheimer's disease & 3 & 3 \\
\hline Parkinson's disease & 1 & 0 \\
\hline Amyotrophic lateral sclerosis (ALS) & 3 & 0 \\
\hline Huntington's disease & 7 & 2 \\
\hline Dentatorubropallidoluysian atrophy & 1 & 0 \\
\hline Prion diseases & 2 & 0 \\
\hline \multicolumn{3}{|l|}{ Metabolic Disorders } \\
\hline Type I diabetes mellitus & 4 & 3 \\
\hline Type II diabetes mellitus & 3 & 0 \\
\hline Maturity onset diabetes of the young & 2 & 1 \\
\hline Total Number of Genes & 532 & 142 \\
\hline
\end{tabular}


Table 6.2: Paracrine response genes.

\begin{tabular}{|c|c|c|c|c|}
\hline $\begin{array}{l}\text { Entrez } \\
\text { Gene ID }\end{array}$ & Symbol & FC & P-value & Name \\
\hline 103406 & $\begin{array}{l}9130206 \\
\text { N08Rik }\end{array}$ & 1.26 & 0.0428 & RIKEN cDNA 9130206N08 gene \\
\hline 12526 & Cd8b1 & -1.35 & 0.00791 & CD8 antigen, beta chain 1 \\
\hline 12611 & Cebpg & -1.35 & 0.00249 & $\begin{array}{l}\text { CCAAT/enhancer binding protein (C/EBP), } \\
\text { gamma }\end{array}$ \\
\hline 12946 & Crry & 1.31 & 0.0298 & complement receptor related protein \\
\hline 13610 & Edg3 & -1.28 & 0.0336 & $\begin{array}{l}\text { endothelial differentiation, sphingolipid } \\
\text { G-protein-coupled receptor, } 3\end{array}$ \\
\hline 14825 & Cxcl1 & 4.84 & 0.00188 & chemokine (C-X-C motif) ligand 1 \\
\hline 14964 & H2-D1 & 1.53 & 0.00849 & histocompatibility 2, D region locus 1 \\
\hline 14972 & $\mathrm{H} 2-\mathrm{K} 1$ & 1.25 & 0.00245 & histocompatibility $2, \mathrm{~K} 1, \mathrm{~K}$ region \\
\hline 14980 & $\mathrm{H} 2-\mathrm{L}$ & 1.24 & 0.0156 & histocompatibility 2, D region \\
\hline 15216 & $\mathrm{Hfe}$ & 1.18 & 0.022 & hemochromatosis \\
\hline 15285 & Hlxb9 & 1.31 & 0.0449 & homeobox gene HB9 \\
\hline 15531 & Ndst1 & 1.19 & 0.0205 & $\begin{array}{l}\mathrm{N} \text {-deacetylase/ } \mathrm{N} \text {-sulfotransferase (heparan } \\
\text { glucosaminyl) } 1\end{array}$ \\
\hline 15900 & Irf8 & -1.41 & 0.0263 & interferon regulatory factor 8 \\
\hline 15945 & Cxcl10 & 2.07 & 0.00616 & chemokine (C-X-C motif) ligand 10 \\
\hline 16071 & lgk-C & 1.52 & 0.00281 & $\begin{array}{l}\text { immunoglobulin kappa chain, constant } \\
\text { region }\end{array}$ \\
\hline 16160 & $\| 12 b$ & 1.30 & 0.00497 & interleukin 12b \\
\hline 17084 & Ly86 & -1.41 & 0.011 & lymphocyte antigen 86 \\
\hline 17319 & Mif & 1.78 & 0.00832 & macrophage migration inhibitory factor \\
\hline 17857 & $\mathrm{M} \times 1$ & 1.53 & 0.0168 & myxovirus (influenza virus) resistance 1 \\
\hline 17969 & Ncf1 & 1.23 & 0.00332 & neutrophil cytosolic factor 1 \\
\hline 18636 & Cfp & -2.04 & 0.00102 & complement factor properdin \\
\hline 20293 & Ccl12 & 6.96 & 0.00148 & chemokine (C-C motif) ligand 12 \\
\hline 20296 & Ccl2 & 1.6 & 0.00123 & chemokine (C-C motif) ligand 2 \\
\hline 20300 & Ccl25 & -1.19 & 0.0373 & chemokine (C-C motif) ligand 25 \\
\hline 20315 & Cxcl12 & -1.56 & 0.00135 & chemokine (C-X-C motif) ligand 12 \\
\hline 22637 & Zap70 & 1.18 & 0.0471 & zeta-chain (TCR) associated protein kinase \\
\hline 22695 & Zfp36 & 1.63 & $8.39 \mathrm{e}-05$ & zinc finger protein 36 \\
\hline 24088 & TIr2 & 1.65 & 0.00744 & toll-like receptor 2 \\
\hline 27205 & Podxl & -1.12 & 0.0156 & podocalyxin-like \\
\hline 330122 & Gm1960 & 4.09 & 0.00683 & gene model 1960, (NCBI) \\
\hline 56298 & Arl6ip2 & 1.22 & 0.0201 & $\begin{array}{l}\text { ADP-ribosylation factor-like } 6 \text { interacting } \\
\text { protein } 2\end{array}$ \\
\hline 56480 & Tbk1 & 1.50 & 0.0305 & TANK-binding kinase 1 \\
\hline 630509 & $\mathrm{H} 2-\mathrm{Q} 7$ & 1.49 & 0.0186 & $\begin{array}{l}\text { similar to } \mathrm{H}-2 \text { class I histocompatibility } \\
\text { antigen, Q7 alpha chain precursor }\end{array}$ \\
\hline 71735 & $\begin{array}{c}1200011 \\
\text { O22Rik }\end{array}$ & -1.67 & 0.00539 & RIKEN cDNA 1200011022 gene \\
\hline 72699 & Lime1 & -1.25 & 0.00332 & Lck interacting transmembrane adaptor 1 \\
\hline 80859 & Nfkbiz & 1.68 & 0.019 & $\begin{array}{l}\text { nuclear factor of kappa light polypeptide } \\
\text { gene enhancer in B-cells inhibitor, zeta }\end{array}$ \\
\hline
\end{tabular}


Table 6.3: Paracrine response pathways.

\begin{tabular}{|c|c|c|}
\hline KEGG Pathway & \# of Genes & Entrez Gene IDs \\
\hline $\begin{array}{l}\text { Cytokine-cytokine receptor } \\
\text { interaction }\end{array}$ & 7 & $\begin{array}{l}1482515945161602029320296 \\
2030020315\end{array}$ \\
\hline $\begin{array}{l}\text { Toll-like receptor signaling } \\
\text { pathway }\end{array}$ & 4 & 15945161602408856480 \\
\hline Type I diabetes mellitus & 3 & 149641497216160 \\
\hline $\begin{array}{l}\text { Natural killer cell mediated } \\
\text { cytotoxicity }\end{array}$ & 3 & 149641497222637 \\
\hline $\begin{array}{l}\text { Cell adhesion molecules } \\
\text { (CAMs) }\end{array}$ & 3 & 125261496414972 \\
\hline $\begin{array}{l}\text { Antigen processing and } \\
\text { presentation }\end{array}$ & 3 & 125261496414972 \\
\hline $\begin{array}{l}\text { Leukocyte transendothelial } \\
\text { migration }\end{array}$ & 2 & 1796920315 \\
\hline $\begin{array}{l}\text { T cell receptor signaling } \\
\text { pathway }\end{array}$ & 2 & 1252622637 \\
\hline Hematopoietic cell lineage & 1 & 12526 \\
\hline Jak-STAT signaling pathway & 1 & 16160 \\
\hline $\begin{array}{l}\text { Complement and coagulation } \\
\text { cascades }\end{array}$ & 1 & 12946 \\
\hline Axon guidance & 1 & 20315 \\
\hline $\begin{array}{l}\text { Neuroactive ligand-receptor } \\
\text { interaction }\end{array}$ & 1 & 13610 \\
\hline $\begin{array}{l}\text { Glycan structures - } \\
\text { biosynthesis } 1\end{array}$ & 1 & 15531 \\
\hline Heparan sulfate biosynthesis & 1 & 15531 \\
\hline $\begin{array}{l}\text { Maturity onset diabetes of the } \\
\text { young }\end{array}$ & 1 & 15285 \\
\hline Tyrosine metabolism & 1 & 17319 \\
\hline Phenylalanine metabolism & 1 & 17319 \\
\hline
\end{tabular}


immune cells to a site of damage. The chemokines are known to be chemoattractants for leukocytes, monocytes, and neutrophils, a well as other effector cells.

Of the six chemokines, Ccl12 (Chemokine ( $\mathrm{C}-\mathrm{C}$ motif) ligand 12) and $\mathrm{Cxcl} 1$ (Chemokine (C-X-C motif) ligand 1) have the largest increase at 2-hrs after radiation. Ccl12 showed a fold change of 6.96 , while Cxcl1 showed a fold change of 4.84 over the unirradiated. It is thought, that $\mathrm{Ccl} 12$ coordinates movement of eosinophils, monocytes, and lymphocytes during an immune response [135]. Cxcl1 has been shown to be a chemoattractant for neutrophils, and to be involved in the processes of inflammation, wound healing, and angiogenesis. In addition, $\mathrm{Cxcl} 1$ has been shown to control the positioning of oligodendrocyte precursors in the developing spinal cord by stopping controlling their migration [136].

\subsection{Vascular versus parenchymal}

Distinguishing the difference between vascular and parenchymal response is very difficult because ultimately they are entwined with one effecting the other. However, there are specific responses that are predominantly observed in each of the two populations. Tables 6.4 and 6.5 show genes that are directly related to vessels/vasculature and neurons, respectively. The breakdown of these genes into functional KEGG pathways is shown in Tables 6.6 and 6.7. There were significantly altered 18 genes identified as part of the vascular response and 36 identified as part of the parenchymal response. Of the genes identified for the vascualr response, the predominate pathways altered were cell adhesion molecules and leukocyte transendothelial migration. In the genes identified as part of the parenchymal response, the most altered pathways were: neuroactive ligand-receptor interaction, MAPK signaling, and axon guidance. Two genes of interest from these groups are Pdgfra (platelet derived growth factor receptor, alpha polypeptide) from the vascular response and Hes5 (hairy and enhancer of split 5) from the parenchymal response.

Pdgfra is a growth factor that regulates cell growth and division. It also been shown to be important in the formation of blood vessels (angiogenesis), as well as cell migration and cell proliferation. When pdgfra is activated, it has been shown to activate several signal transduction pathways including MAPK and PI3K. Following cranial radiation, MAPK has the largest number of significantly altered genes. At 2-hrs after cranial radiation, pdgfra had a fold change of 1.48 .

Hes 5, along with Hes 1 and Hes 3, has been shown to affect the differentiation patterns of neuronal stem cells. Hatakeyama et al have shown that the Hes genes are critical to the proper development (i.e., size, shape, and cell arrangement) of brain structures by controlling when neuronal stem cells differentiate. At 2-hrs after cranial radiation, the Hes 5 gene had a fold change of -2.22 . If this gene is down-regulated, it could have a significant impact in a developing brain.

\subsection{Early signs of neurostructural changes}

Numerous studies have shown that years out from cranial radiation, patients experience neurological deficits. This is in part due to necrosis of both the white and gray matter, 
Table 6.4: Genes related to the response of the vasculature.

\begin{tabular}{|c|c|c|c|c|}
\hline $\begin{array}{l}\text { Entrez } \\
\text { Gene ID }\end{array}$ & Symbol & $\mathrm{FC}$ & P-value & Name \\
\hline 107746 & Rapgef1 & 1.21 & 0.0207 & $\begin{array}{l}\text { Rap guanine nucleotide exchange factor } \\
\text { (GEF) } 1\end{array}$ \\
\hline 11998 & Avp & -1.15 & 0.0347 & arginine vasopressin \\
\hline 12387 & Ctnnb1 & 1.63 & 0.0249 & catenin (cadherin associated protein), beta 1 \\
\hline 15214 & Hey2 & -1.35 & 0.0146 & $\begin{array}{l}\text { hairy/enhancer-of-split related with YRPW } \\
\text { motif } 2\end{array}$ \\
\hline 16007 & Cyr61 & 2.76 & 0.000207 & cysteine rich protein 61 \\
\hline 17123 & Madcam1 & -1.43 & 0.0215 & $\begin{array}{l}\text { mucosal vascular addressin cell adhesion } \\
\text { molecule } 1\end{array}$ \\
\hline 17967 & Ncam1 & -1.67 & 0.003 & neural cell adhesion molecule 1 \\
\hline 18007 & Neo1 & -1.32 & 0.0363 & neogenin \\
\hline 18186 & Nrp1 & -1.41 & 0.00267 & neuropilin 1 \\
\hline 18212 & Ntrk2 & -1.47 & 0.00595 & $\begin{array}{l}\text { neurotrophic tyrosine kinase, receptor, type } \\
2\end{array}$ \\
\hline 18595 & Pdgfra & 1.48 & 0.0192 & $\begin{array}{l}\text { platelet derived growth factor receptor, alpha } \\
\text { polypeptide }\end{array}$ \\
\hline 19317 & Qk & -1.64 & 0.00963 & quaking \\
\hline 20315 & Cxcl12 & -1.56 & 0.00135 & chemokine ( $\mathrm{C}-\mathrm{X}-\mathrm{C}$ motif) ligand 12 \\
\hline 218397 & Rasa1 & -1.22 & 0.0276 & RAS p21 protein activator 1 \\
\hline 22329 & Vcam1 & 1.26 & 0.0365 & vascular cell adhesion molecule 1 \\
\hline 56458 & Foxo1 & 1.19 & 0.00517 & forkhead box $\mathrm{O} 1$ \\
\hline 56632 & Sphk2 & -1.33 & 0.0133 & sphingosine kinase 2 \\
\hline 67916 & Ppap2b & -1.37 & 0.018 & phosphatidic acid phosphatase type $2 \mathrm{~B}$ \\
\hline
\end{tabular}


Table 6.5: Genes related to the response of the parenchyma.

\begin{tabular}{|c|c|c|c|c|}
\hline $\begin{array}{l}\text { Entrez } \\
\text { Gene ID }\end{array}$ & Symbol & FC & P-value & Name \\
\hline 107934 & Celsr3 & -1.23 & 0.0245 & $\begin{array}{l}\text { cadherin EGF LAG seven-pass G-type } \\
\text { receptor } 3\end{array}$ \\
\hline 109648 & Npy & -1.33 & 0.0449 & neuropeptide $Y$ \\
\hline 11441 & Chrna7 & 1.39 & 0.0376 & $\begin{array}{l}\text { cholinergic receptor, nicotinic, alpha } \\
\text { polypeptide } 7\end{array}$ \\
\hline 116837 & Rims1 & -1.28 & 0.0107 & $\begin{array}{l}\text { regulating synaptic membrane } \\
\text { exocytosis } 1\end{array}$ \\
\hline 11820 & App & -2.08 & 0.00772 & amyloid beta $(\mathrm{A} 4)$ precursor protein \\
\hline 11906 & Atbf1 & -1.25 & 0.0259 & AT motif binding factor 1 \\
\hline 11998 & Avp & -1.12 & 0.0347 & arginine vasopressin \\
\hline 12064 & Bdnf & -1.45 & 0.0278 & brain derived neurotrophic factor \\
\hline 12394 & Runx1 & 1.32 & 0.042 & runt related transcription factor 1 \\
\hline 12426 & Cckbr & -1.35 & 0.0092 & cholecystokinin B receptor \\
\hline 12608 & Cebpb & 1.38 & 0.0408 & $\begin{array}{l}\text { CCAAT/enhancer binding protein } \\
\text { (C/EBP), beta }\end{array}$ \\
\hline 12704 & Cit & -1.19 & 0.0355 & citron \\
\hline 13175 & Dcamkl1 & 1.54 & 0.0436 & $\begin{array}{l}\text { doublecortin and } \\
\text { calcium/calmodulin-dependent protein } \\
\text { kinase-like } 1\end{array}$ \\
\hline 13176 & Dcc & -1.64 & 0.00485 & deleted in colorectal carcinoma \\
\hline 13385 & Dlgh4 & 1.84 & 0.0027 & discs, large homolog 4 (Drosophila) \\
\hline 13797 & Emx2 & -1.23 & 0.0212 & $\begin{array}{l}\text { empty spiracles homolog } 2 \\
\text { (Drosophila) }\end{array}$ \\
\hline 140919 & Slc17a6 & 1.51 & 0.0371 & $\begin{array}{l}\text { solute carrier family } 17 \\
\text { (sodium-dependent inorganic } \\
\text { phosphate cotransporter), member } 6\end{array}$ \\
\hline 14291 & Fpr-rs4 & 1.17 & 0.0474 & $\begin{array}{l}\text { formyl peptide receptor, related } \\
\text { sequence } 4\end{array}$ \\
\hline 14395 & Gabra2 & -1.47 & 0.0329 & $\begin{array}{l}\text { gamma-aminobutyric acid (GABA-A) } \\
\text { receptor, subunit alpha } 2\end{array}$ \\
\hline 15208 & Hes5 & -2.22 & 0.000678 & $\begin{array}{l}\text { hairy and enhancer of split } 5 \\
\text { (Drosophila) }\end{array}$ \\
\hline 170757 & Eltd1 & 1.12 & 0.0382 & $\begin{array}{l}\text { EGF, latrophilin seven transmembrane } \\
\text { domain containing } 1\end{array}$ \\
\hline 18189 & Nrxn1 & 1.72 & 0.033 & neurexin I \\
\hline 18222 & Numb & -1.75 & 0.00514 & numb gene homolog ( \\
\hline 18227 & $\mathrm{Nr} 4 \mathrm{a} 2$ & -1.61 & 0.037 & $\begin{array}{l}\text { nuclear receptor subfamily } 4 \text {, group } \mathrm{A} \text {, } \\
\text { member } 2\end{array}$ \\
\hline 192678 & Rassf3 & 1.23 & 0.0319 & $\begin{array}{l}\text { Ras association (RalGDS/AF-6) } \\
\text { domain family } 3\end{array}$ \\
\hline 19732 & Rgl2 & -1.19 & 0.0302 & $\begin{array}{l}\text { ral guanine nucleotide dissociation } \\
\text { stimulator-like } 2\end{array}$ \\
\hline 19877 & Rock1 & -1.25 & 0.0459 & $\begin{array}{l}\text { Rho-associated coiled-coil containing } \\
\text { protein kinase } 1\end{array}$ \\
\hline
\end{tabular}


Table 6.5: (continued).

\begin{tabular}{ccccl}
\hline $\begin{array}{c}\text { Entrez } \\
\text { Gene ID }\end{array}$ & Symbol & FC & P-value & Name \\
\hline 20320 & Nptn & -1.27 & 0.0479 & neuroplastin \\
20979 & Syt1 & -1.33 & 0.0128 & synaptotagmin I \\
215690 & Nav1 & -1.23 & 0.00842 & neuron navigator 1 \\
21812 & Tgfbr1 & 1.25 & 0.0273 & transforming growth factor, beta \\
& & & & receptor I \\
218397 & Rasa1 & -1.22 & 0.0276 & RAS p21 protein activator 1 \\
21974 & Top2b & 1.70 & 0.0344 & topoisomerase (DNA) II beta \\
225870 & Rin1 & -1.14 & 0.0401 & Ras and Rab interactor 1 \\
319387 & Lphn3 & 1.36 & 0.00477 & latrophilin 3 \\
381677 & Vgf & -1.25 & 0.0053 & VGF nerve growth factor inducible \\
\hline
\end{tabular}

Table 6.6: Pathway classification of the genes related to the vascular response.

\begin{tabular}{lcl}
\hline KEGG pPathway & \# of Genes & Entrez Gene IDs \\
\hline $\begin{array}{l}\text { Cell adhesion molecules } \\
\text { (CAMs) }\end{array}$ & 4 & 17123179671800722329 \\
$\begin{array}{l}\text { Leukocyte transendothelial } \\
\text { migration }\end{array}$ & 3 & 123872031522329 \\
$\begin{array}{l}\text { MAPK signaling pathway } \\
\text { Focal adhesion }\end{array}$ & 3 & 1821218595218397 \\
$\begin{array}{l}\text { Axon guidance } \\
\text { Colorectal cancer }\end{array}$ & 3 & 1077461238718595 \\
$\begin{array}{l}\text { Cytokine-cytokine receptor } \\
\text { interaction }\end{array}$ & 2 & 1818620315218397 \\
$\begin{array}{l}\text { Sphingolipid metabolism } \\
\text { Insulin signaling pathway }\end{array}$ & 2 & 1859520315 \\
Calcium signaling pathway & 2 & 5663267916 \\
Huntington's disease & 2 & 10774656458 \\
Regulation of actin & 2 & 1859556632 \\
cytoskeleton & 1 & 218397 \\
Gap junction & 1 & 18595 \\
$\begin{array}{l}\text { Tight junction } \\
\text { Adherens junction }\end{array}$ & 1 & 18595 \\
VEGF signaling pathway & 1 & 12387 \\
Wnt signaling pathway & 1 & 12387 \\
Neuroactive ligand-receptor & 1 & 56632 \\
interaction & 1 & 12387 \\
Ether lipid metabolism & 11998 \\
\hline
\end{tabular}


Table 6.7: Pathway classification of the genes related to the parenchymal response.

\begin{tabular}{lcl}
\hline KEGG Pathway & \# of Genes & Entrez Gene IDs \\
\hline Neuroactive ligand-receptor interaction & 4 & 109648119981242614395 \\
MAPK signaling pathway & 3 & 1206421812218397 \\
Axon guidance & 3 & 1317619877218397 \\
Chronic myeloid leukemia & 2 & 1239421812 \\
Calcium signaling pathway & 2 & 1144112426 \\
TGF-beta signaling pathway & 2 & 1987721812 \\
Huntington's disease & 2 & 12064218397 \\
Colorectal cancer & 2 & 1317621812 \\
Notch signaling pathway & 2 & 1520818222 \\
Pancreatic cancer & 1 & 21812 \\
Alzheimer's disease & 1 & 11820 \\
Adipocytokine signaling pathway & 1 & 109648 \\
Regulation of actin cytoskeleton & 1 & 19877 \\
Leukocyte transendothelial migration & 1 & 19877 \\
Adherens junction & 1 & 21812 \\
Wnt signaling pathway & 1 & 19877 \\
Cytokine-cytokine receptor interaction & 1 & 21812 \\
Neurodegenerative Disorders & 1 & 11820 \\
Focal adhesion & 1 & 19877 \\
\hline
\end{tabular}


vasculopathy, and the inhibition of neurogenesis. Clinically, signs are not seen until months to years following radiation therapy, however by looking at the changes in gene expression acutely after radiation, we can see that there are neurostructural changes in the acute phase that could potentially be a cause for the late changes. By evaluating these changes further, there is the potential for therapeutic intervention in the early stage that could reduce the long-term effects. Table 6.8 shows the genes that are significantly different 2 -hrs after radiation and are known to be related to developmental and neurodegenerative diseases. There are 34 genes that are significantly altered compared to the unirradiated.

One gene of interest related to neurodegenerative diseases, is amyloid precursor protein (APP). At 2-hrs after a single dose of 20-Gy radiation, APP has a fold change of -2.08. APP generates amyloid beta amino acid peptides, a main component of the amyloid plaques found in Alzheimer's disease. APP regulates many neuronal functions including synapse formation, cell excitability, synaptic transmission, long-perm potentiation and cell adhesion [137, 138, 139, 140, 141]. In Alzheimer's disease, the amount of APP in a cell decreases in proximity to an amyloid plaque and the decrease may contribute to dimentia, a hallmark of Alzheimer's disease. Recent work has also suggested that the level of APP affects behavioral learning and memory[139, 140, 141].

\subsection{Genes of interest}

Out of the many genes evaluated at 2-hrs after cranial irradiation, there are some genes of interest that were not included in the analysis above, but are of interest in their relation to inflammation and/or radiation response. ICAM-1, which was investigated in this work as a radiation-induced inflammatory molecule, showed a fold change of 2.53, while GFAP (measured to evaluate astrocyte activation) had a fold change of 1.09. The fold change of GFAP is not unexpected, as immunohistochemistry staining at 2-hrs after radiation showed no significant increase in activated astrocytes. HIF- $1 \alpha$, a hypoxia marker that is known to be up-regulated following radiation [142, 143, 144], showed a fold increase of 1.89, while BCL-2 (B-cell leukemia/lymphoma 2) an anti-apoptotic molecule[145, 146], was shown to be down-regulated (fold change of -1.43) at 2-hrs post-irradiation, which correlates with the increase in p53 apoptosis observed following radiation. ATM (ataxia telangiectasia mutated) is a gene that is commonly associated with radiation damage and it has been shown that cellular radiosensitivity is increased in its absence, only showed a fold changes of 1.07 [147, 148, 149, 150]. This is not unexpected as the effects of ATM have been seen following radiation without an actual alteration in the expression of ATM $[148,150]$. Another gene of interest is Arc (activity-regulated cytoskeletal-related gene), which has been associated with learning and memory formation, and showed a fold change of -3.97 . Studies have shown that radiation can alter Arc expression and that this may correlate with the learning deficits observed following cranial irradiation [151, 152, 153]. 
Table 6.8: Modulated genes related to development and neurodegenerative diseases.

\begin{tabular}{|c|c|c|c|c|}
\hline $\begin{array}{l}\text { Entrez } \\
\text { Gene ID }\end{array}$ & Symbol & FC & P-value & Name \\
\hline 108100 & Baiap2 & -1.25 & 0.0328 & $\begin{array}{l}\text { brain-specific angiogenesis inhibitor } \\
\text { 1-associated protein } 2\end{array}$ \\
\hline 11820 & App & -2.08 & 0.00772 & amyloid beta (A4) precursor protein \\
\hline 12043 & $\mathrm{Bcl} 2$ & -1.43 & 0.0259 & B-cell leukemia/lymphoma 2 \\
\hline 12064 & Bdnf & -1.45 & 0.0278 & brain derived neurotrophic factor \\
\hline 12914 & Crebbp & -1.69 & 0.0125 & CREB binding protein \\
\hline 13176 & Dcc & -1.64 & 0.00485 & deleted in colorectal carcinoma \\
\hline 13649 & Egfr & -1.23 & 0.0342 & epidermal growth factor receptor \\
\hline 13835 & Epha1 & 1.31 & 0.0152 & Eph receptor A1 \\
\hline 13841 & Epha7 & 1.48 & 0.00446 & Eph receptor A7 \\
\hline 140499 & Ube2j2 & 1.20 & 0.0125 & $\begin{array}{l}\text { ubiquitin-conjugating enzyme E2, J2 } \\
\text { homolog (yeast) }\end{array}$ \\
\hline 14270 & Srgap2 & -1.20 & 0.0143 & SLIT-ROBO Rho GTPase activating protein 2 \\
\hline 14828 & Hspa5 & -1.47 & 0.0119 & $\begin{array}{l}\text { heat shock } 70 \mathrm{kD} \text { protein } 5 \\
\text { (glucose-regulated protein) }\end{array}$ \\
\hline 15194 & Hdh & 1.25 & 0.0319 & Huntington disease gene homolog \\
\hline 16956 & Lpl & 1.41 & 0.00548 & lipoprotein lipase \\
\hline 16971 & Lrp1 & -1.39 & 0.00421 & $\begin{array}{l}\text { low density lipoprotein receptor-related } \\
\text { protein } 1\end{array}$ \\
\hline 17973 & Nck1 & -1.33 & 0.00739 & $\begin{array}{l}\text { non-catalytic region of tyrosine kinase } \\
\text { adaptor protein } 1\end{array}$ \\
\hline 18176 & Nras & -1.19 & 0.0484 & neuroblastoma ras oncogene \\
\hline 18186 & Nrp1 & -1.41 & 0.00267 & neuropilin 1 \\
\hline 20315 & Cxcl12 & -1.56 & 0.00135 & chemokine (C-X-C motif) ligand 12 \\
\hline 20346 & Sema3a & 1.49 & 0.00176 & $\begin{array}{l}\text { sema domain, immunoglobulin domain (Ig), } \\
\text { short basic domain, secreted, (semaphorin) } \\
\text { 3A }\end{array}$ \\
\hline 20348 & Sema3c & 1.19 & 0.0182 & $\begin{array}{l}\text { sema domain, immunoglobulin domain (Ig), } \\
\text { short basic domain, secreted, (semaphorin) } \\
3 \mathrm{C}\end{array}$ \\
\hline 20564 & Slit3 & -1.54 & 0.00242 & slit homolog 3 (Drosophila) \\
\hline 20662 & Sos 1 & 2.37 & 0.0227 & Son of sevenless homolog 1 (Drosophila) \\
\hline 20832 & Ssr4 & -1.11 & 0.0393 & signal sequence receptor, delta \\
\hline 215114 & Hip1 & -1.19 & 0.00738 & huntingtin interacting protein 1 \\
\hline 218397 & Rasa1 & -1.22 & 0.0276 & RAS p21 protein activator 1 \\
\hline 22253 & Unc5c & -1.23 & 0.0265 & unc-5 homolog C (C. elegans) \\
\hline 23871 & Ets1 & -1.61 & 0.0289 & E26 avian leukemia oncogene 1,5 ' domain \\
\hline 268902 & Robo2 & -1.23 & 0.0316 & roundabout homolog 2 (Drosophila) \\
\hline 319713 & Ablim3 & -1.37 & 0.0121 & actin binding LIM protein family, member 3 \\
\hline 53323 & Hip2 & 1.36 & 0.0447 & huntingtin interacting protein 2 \\
\hline 66725 & Lrrk2 & -1.19 & 0.0109 & leucine-rich repeat kinase 2 \\
\hline 67300 & Cltc & -1.30 & 0.00303 & clathrin, heavy polypeptide (Hc) \\
\hline 74018 & Als2 & -1.20 & 0.0421 & $\begin{array}{l}\text { amyotrophic lateral sclerosis } 2 \text { (juvenile) } \\
\text { homolog (human) }\end{array}$ \\
\hline
\end{tabular}




\subsection{Cytokine analysis}

The serum cytokine G-CSF (granulocyte colony-stimulating factor) showed a significant difference at 15-days post-RT $(P<0.05)$ compared to unirradiated, but not at any other time point measured, see Table 6.9. TNF, VEGF, IL-6, and IL-10 did not show any significant difference at any time point. In a recent study by Merchant et al, cytokines were measured in an attempt to find a clinical biomarker that would predict normal tissue and tumor response to post-operative radiation therapy, in children with ependymoma's [154]. Figure 6.2 shows the correlation between treatment success and treatment failure by looking at the levels of VEGF. This study by Merchant et al [154]shows that cytokines could make a viable marker for evaluating radiation response even though the current study did not show significant changes at the expected time points.

\subsection{Discussion}

This study generated the data to look at the gene expression profile in the mouse brain at 2-hrs after 20-Gy localized cranial irradiation. The large amount of data generated provides the opportunity for further analysis to be carried out, in addition to providing insight into specific questions regarding early markers of radiation damage.

- Is there a paracrine response that occurs?

There is a paracrine response observed following cranial irradiation that is primarily initiated by chemotactic cytokines through the cytokine-cytokine receptor interaction pathway. It is also possible that serum cytokine measurements can be used in the future as a clinical marker of radiation damage/outcome/prognosis.

- Can we distinguish between parenchymal and vascular damage?

The gene expression profiles show that there is damage to both vascular and parenchymal damage following cranial irradiation, and thru the analysis performed using the known functions of the genes, the separate response of the two regions can be distinguished.

- Are there early signs of neurostructural damage?

The results identify 34 genes that were significantly altered at 2-hrs post-irradiation that are known to be related to neurological/neurodegenerative diseases. By identifying these genes, we may begin to understand the underlying reason for the observed cognitive deficits following irradiation that may be the key to limiting these deficits, if properly treated. 
Table 6.9: Serum cytokine results.

\begin{tabular}{ccccccc}
\hline & Control & $24 \mathrm{hrs}$ & $48 \mathrm{hrs}$ & $72 \mathrm{hrs}$ & 8 days & 15 days \\
\hline IL6 & 79 & 111 & 65 & 55 & 141 & 264 \\
p-value & & .9951 & 1.0000 & .9993 & .9610 & .4909 \\
\hline IL10 & 66 & 102 & 60 & 56 & 33 & 66 \\
p-value & & .8242 & .9992 & .9975 & .6430 & 1.0000 \\
\hline GCSF & 133 & 776 & 81 & 160 & 784 & 1656 \\
p-value & & .5257 & 1.0000 & 1.0000 & .5926 & $.0269^{\star}$ \\
\hline VEFG & 99 & 99 & 106 & 107 & 93 & 114 \\
p-value & & 1.0000 & .9684 & .9552 & .9722 & .6191 \\
\hline TNF & 171 & 168 & 169 & 171 & 176 & 191 \\
p-value & 171 & .9100 & .9772 & 1.0000 & .9311 & .1449 \\
\hline
\end{tabular}

Following RT, there was not a significant change in serum cytokines measured up to 15days post-RT, except in GCSF. * indicates a significant difference compared to control.

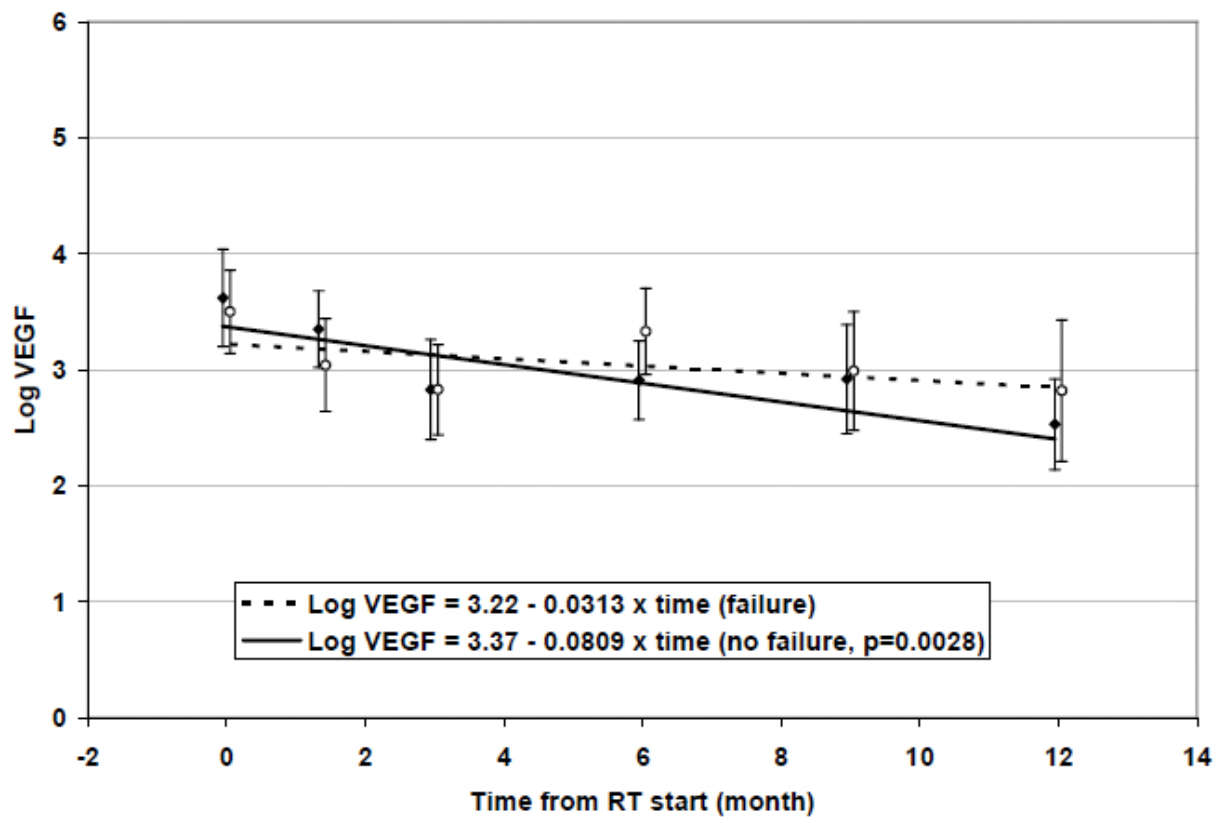

Figure 6.2: Use of cytokine levels as a clinical biomarker.

It was observed tht there is an association between the lack of treatment failure and declining serum VEGF concentration. The graph is a plot of the log VEGF by treatment failure. The observations plotted are local sample mean and standard error. Graph from Merchant et al. "Cytokine and Growth Factor Responses After Radiotherapy for Localized Ependymoma", Int J Radiat Oncol Biol Phys, in print. Copyright (c) 2008 Elsevier, with permission from the publisher. 


\section{Chapter 7}

\section{Discussion}

In this work, we investigated the role of the inflammatory molecules TNF and ICAM-1 in the acute response to localized cranial radiation and investigated the use of an interventional therapy and its ability to abrogate this acute inflammatory response. We also evaluate the changes in the complete gene expression profile to verify a paracrine response, to delineate the damage to the vasculature versus the parenchyma, and to identify early signs of neurostructural damage following cranial radiation.

Our study shows that localized cranial radiation causes a measurable increase in astrogliosis, vessel permeability, leukocyte adhesion, and arteriole vasoconstriction up to 48-hrs after a single dose of 20-Gy radiation that is abrogated when TNF or ICAM-1 is inhibited. We also show that KZ-41 has the ability to abrogate the vascular changes following radiation, but does not have an effect on the astrocytic response. By evaluating the gene expression profile, we can show that there is a paracrine response following radiation that is primarily driven by chemokines, that there is both a vascular and parenchymal response, and that there are multiple genes up-regulated by radiation that are known to be related to neurodegenerative diseases.

\subsection{Radiation-induced vascular changes}

In the current work, we observed an increase in permeability of the BBB and in leukocyte adhesion following radiation that is abrogated when treated with an anti-inflammatory agent (Figures 4.1 and 4.2). Further, arteriole vasoconstriction was observed at 48-hrs post-radiation that was inhibited with treatment (Figure 4.4). Previous studies, in rats, have shown that irradiation induces an increase in BBB permeability that was abrogated following treatment with ICAM-1 mAb [9]. In addition, Panes et al observed an increase in extravasation of FITC-albumin at 6-hrs after radiation in a rat messenteric model [26]. Our present study shows that this increase in BBB permeability following radiation can be abrogated if the inflammatory cytokine TNF or the adhesion molecule ICAM-1 is inhibited. This increased permeability is caused in part by endothelial cell damage, as expressed by changes in tight junction integrity, and by vesicle formation post-irradiation. Radiation-induced damage to endothelial cells has been examined by several investigators $[49,50,51]$ aiming to elucidate the role of this damage in initiating and/or sustaining radiation side effects. In previous studies using electron microscopy we show that radiation causes damage to the tight junctions [70], which is also connected to the observed increase in BBB permeability. In addition, several studies, including our own, have shown an increase in BBB permeability and an increase in the number of vesicles following fractionated cranial irradiation $[70,71,72]$. 
Leukocyte adhesion is known to be up-regulated following single dose radiation [9, 16, 26] and has been shown to be accompanied by an increase in BBB permeability. Yuan et al have shown, using antibody-coated microspheres, that ICAM-1 is up-regulated following cranial radiation and reduced when treated with the anti-inflammatory drug dexamethasone [16]. The number of adherent anti-ICAM1 microspheres was five and nine times that of IgG at 24- and 48-hrs post-radiation, respectively, and returned to baseline 7 days post-radiation [16]. It has also been shown that E-selectin, and P-selectin are up-regulated following cranial irradiation $[16,26]$, which, in addition to ICAM-1, have been shown to be up-regulated by TNF. In our study, we show that when treated with anti-TNF or anti-ICAM1 , radiation-induced leukocyte adhesion can be abrogated, which shows that both of these molecules play an active role in this process. We also show that there is a significant correlation between the permeability of the BBB and leukocyte adhesion and that if one effect is limited, the other will be reduced as well.

Our study also shows that radiation induces a decrease in arteriole diameter that is mediated by TNF, as our rTNF results demonstrated. The mechanisms of vasoconstriction are not completely understood, but include: alterations in calcium signaling from the astrocytes, changes in nitric oxide levels, constriction of the smooth muscle cells, hematocrit variations, and over-proliferation of endothelial cells [81, 17, 82, 83, 84]. The reduction in arteriole diameter observed correlates with a study by Ansari et al [155] who shows that at 72-hrs post-20-Gy radiation there is an increase in hypoxia, which can be inhibited when treated with TNF mAb. The observed decrease in arteriole diameter at 48-hrs post- radiation in our study could play a role in initiating the hypoxia that Ansari et al observed at 72-hrs post-radiation [155]. In addition, it has been shown that ionizing radiation can induce changes in microvascular structure and function $[10,11,156]$. Previous studies have shown $[10,156]$ that radiation significantly changes the structure and microhemodynamics of capillaries in the hamster cremaster muscle: compared to age-matched controls at early time points $(3,7$, and 30 days post-irradiation). Vessel diameter significantly decreased with age, red blood cell velocity was significantly less, capillary surface area decreased and vessel hematocrit increased, and finally blood flow was significantly lower. At late time points $(60,120$, and 180 days post-irradiation) red blood cell flux, red blood cell velocity and capillary blood flow in networks at the 180 day time point were significantly larger than age-matched controls. This indicates that the radiation-induced microvascular changes are different in the late-term versus the acute, however, we believe that there is persistent acute damage that eventually leads to the late-term effects.

To investigate the specific role of TNF in the observed irradiation-induced leukocyte adhesion and changes in vessel diameter, rTNF was administered systemically. At 7-hrs post-injection, there was a significant increase in leukocyte adhesion in both the venules and arterioles, while at 24-hrs there was only a significant increase in arteriole adhesion. Carvalho-Tavares et al observed similar increases in leukocyte adhesion in brain vasculature with diameters between 50 and $120 \mu \mathrm{m}$ following intraperitoneal administration of TNF [157], while Wright et al observed an increase in leukocyte infiltration at the site of an intracerebral injection of human rTNF in rats [158]. In our study we did not observe any leukocyte adhesion in the cerebral arterioles following radiation treatment. This is in contrast to a study by Acker et al who, in rats, indicates that arterioles show more leukocyte adhesion than venules following collimated cranial irradiation [159]. The difference in results between the present study, and that by Acker et al [159] could be explained by the difference in the size of the arterioles measured. The diameter of the arterioles mea- 
sured by Acker et al was almost twice the size measured in the current study, as it was in rats rather than mice. Outside of the brain, adhesion activity is often seen in arterioles, but with a difference observed between diameters, with larger arterioles having more adhesion than smaller arterioles [160, 161]. In addition, Mayhan used a rat open window model, to show that the presence of TNF, via topical suffusion, did not affect arteriole diameters [162]. Our study disagrees with this finding. The difference in findings is most likely due to topical versus systemic administration of rTNF. We believe that systemic administration is more relevant for comparison to elucidate the role of TNF in the observed radiation-induced arteriole diameter changes.

By inhibiting TNF and ICAM-1, we are attempting to modulate the initial inflammatory response that is created following radiotherapy, in an attempt to reduce the vascular damage and activated astrocytes associated with radiation treatment. By successfully abrogating these changes when treated, we show that these two molecules are at least partly responsible for radiation-induced permeability, leukocyte adhesion changes, and arteriole diameter changes, and are good targets for limiting this damage.

\subsection{Histology}

Our data shows that there is a significant increase in activated astrocytes up to 48hrs post-radiation and that when treated with radiation and anti-TNF or radiation and antiICAM-1, the amount of activated astrocytes is significantly lower (Figure 4.6). In addition, we show that there is a significant difference in the amount of activated astrocytes following radiation when treated with anti-TNF versus anti-ICAM-1. This could imply that there is a signal, possibly NF- $\kappa \mathrm{B}$, above ICAM-1 in the TNF pathway that stimulates the activation of the astrocytes. Others have also shown that radiation causes an inflammatory response in the parenchyma through the activation [47] of astrocytes and microglial cells [163, 152, 69]. With a single dose of 15-Gy, Hwang et al. showed that signs of radiation induced activated astrocytes could be seen as early as 6-hrs post-radiation, though not significant until 24hrs post-radiation [69]. In vitro, Kyrkandies et al show that the irradiating of astrocytes can significantly induce astrocyte activation as early as 4-hrs post-radiation accompanied by an increase in TNF and IL-1 $\beta$ mRNA [58]. In a fractionated radiation model (2-Gy/day for 4 weeks for a total of 40-Gy), astrocyte activation was delayed until 60 days from the start of radiation, but remained significantly increased through 180 days [70]. Activated astrocytes have been shown to have both positive and negative effects in the brain. It has been shown that reactive astrocytes protect neurons from oxidative stress and play a crucial role in the antioxidant defense in the brain via a glutathione dependent mechanism, but also inhibit CNS regeneration and neurogenesis. Previous studies have shown that after CNS injury, the presence of activated astrocytes can inhibit cell proliferation and neuroregeneration [164, 165], and that prolonged activation of astrocytes can create glial scar sites which has been theorized to inhibit axonal regeneration or remyelination [166, 167]. Whether radiation-induced astrocyte activation is caused by direct ionization damage or due to the breach in the BBB exposing the parenchyma to noxious substances and inflammatory cytokines is unclear. However, activated astrocytes have been shown to secrete inflammatory agents $[98,97]$ which, in turn, could amplify microvascular damage. Protect- 
ing the astrocytes from radiation-induced damage may be a key factor in limiting long-term damage in the brain.

We and others have shown that radiation induces the gene expression of TNF and ICAM-1 in the brain $[56,57,168]$. Binding of TNF to its receptors on the endothelial cell surface has been implicated in the activation of NF- $\kappa \mathrm{B}$, which in turn switches on the synthesis of adhesion glycoproteins such as ICAM-1 [80]. ICAM-1 up-regulation can cause increased leukocyte endothelial cell interactions triggering cytoskeletal remodeling, which can be accompanied by paracellular transport [169, 170, 26, 9]. Our immunofluorescence studies show that TNF protein levels are elevated at 24- and 48-hrs post-radiation accompanied by an increase in ICAM-1 protein levels. Treatment with radiation and anti-TNF resulted in no expression of TNF protein and minimal expression of ICAM-1 protein. Treatment with radiation and anti-ICAM resulted in no expression of ICAM-1, but some expression of TNF. This is because TNF expression can be stimulated by molecules other than ICAM-1, such as NF- $\kappa$ B. Therefore, blocking ICAM-1 does not imply that TNF expression levels will be affected. In addition, by administering rTNF, we show that TNF can induce the protein expression of both TNF and ICAM-1. The detection of the increase in protein levels of these two inflammatory molecules has an added significance over the previous findings which were measurements of mRNA levels. Further, these results could offer a mechanistic explanation for the observed increase in BBB permeability and the subsequent inhibition of this increase following treatment with anti-TNF or anti-ICAM-1 and the resulting protein expression levels.

\subsection{The role of TNF}

We have shown that TNF is involved in and is capable of producing the observed acute effects following radiation. We achieve this in two different ways: first, by inducing the effects of TNF by administering recombinant TNF and second, by irradiating and treating with an antibody to TNF hence inhibiting the effect of TNF. The recombinant TNF showed that leukocyte adhesion, arteriole diameter changes and astrogliosis can be induced by TNF as early as 7-hrs after $0.5 \mu \mathrm{g}$ rTNF. By irradiating and treating with anti-TNF, we show that the observed effects induced by irradiation (BBB permeability, leukocyte adhesion, arteriole diameter changes, and astrogliosis) can be abrogated if TNF is inhibited. This shows that there is an acute inflammatory response initiated when localized cranial irradiation is given, and that TNF is a key factor in this response. Currently in the clinic, there are inhibitors to TNF being used to treat chronic inflammatory diseases and autoimmune disorders such as, rheumatoid arthritis, Crohn's disease, psoriasis, and ankylosing spondylitis. Two of these inhibitors are monoclonal antibody-based, infliximab and adalimumab, while the other is a receptor fusion protein, etanercept $[171,172]$. In addition, several anti-TNF agents have been studied for their anti-tumor activity including infliximab, thalidomide, and lenalidomide [173, 174, 175]. Infliximab has been used in rheumatoid arthritis and has recently showed clinical efficacy in a phase II trial against renal cell carcinoma [173]. Thalidomide is a derivative of glutamic acid which has anti-inflammatory and angiogenic activities; it inhibits the production of TNF, VEGF, and decreases the binding activity of NF- $\kappa \mathrm{B}[176,177]$ and is currently in a phase II trial for the treatment of recurrent glioblastoma. Groves et al, show that the combination of temozolomide plus thalidomide 
was safe but with little improvement over temozolomide alone [178]. Lenalidomide or CC5013 is an analog of thalidomide that has shown increased potency in preclinical trials against myelomas and fewer nonhematologic side effects [174, 175]. Both thalidomide and lenalidomide show increased efficacy when used in combination with dexamethasone albeit with higher risk of thrombosis that can be prevented with the use of aspirin [174, 179]. Thalidomide and lenalidomide are small molecules [180] that can cross the BBB in therapeutic quantities and have been shown to significantly increase survival and to decrease motor neuron cell death in transgenic mice with amyotrophic lateral sclerosis symptoms [181].

It is quite practical and probable that introducing the use of an anti-TNF drug to help limit the radiation-induced damage will be evaluated in the future. This work shows that TNF is a main component in the acute vascular damage following cranial radiation, and that there is an inflammatory component to radiation damage that can be easily addressed. With the numerous inhibitors of TNF already available, and the re-evaluation of anti-TNF drugs as tumor treatments, the combination could prove promising. However, the length of time in which TNF is inhibited must be controlled, as Daigle et al have shown that the lack of the TNF p75 receptor following cranial radiation can exacerbate radiation-induced demyelination, in both severity and in location of myelin loss [66].

\section{$7.4 \quad \mathrm{KZ}-41$}

$\mathrm{KZ}-41$ is a novel drug derived from quinic acid that preliminary studies have shown to be a potent anti-inflammatory agent. In vitro studies have indicated that KZ-41 acts through the NF- $\kappa$ B pathway. Our in vivo studies show that $K Z-41$ has the ability to reduce radiation-induced inflammation in the brain following localized cranial irradiation having a similar effect to that of pure antibodies to TNF and ICAM-1. KZ-41 limits radiation-induced increases in BBB permeability, leukocyte adhesion, and arteriole diameter changes. It also limits the expression of TNF and ICAM-1 protein. It does not however inhibit radiationinduced astrogliosis. In fact it actually appears to act as a radiosensitizer to the astrocytes making them more sensitive to the damage from radiation. This is a very interesting characteristic. This is a drug that can protect the vasculature from radiation-induced damage while at the same time increase the sensitivity of parenchymal cells to radiation.

Other studies have shown that nonsteroidal anti-inflammatory drugs (NSAIDs), such as ibuprofen, enhance the sensitivity of cells to radiation [182, 183, 184]. The mechanism by which NSAIDs act as radiosensitizers is not fully understood, however, Palayoor et al have shown that ibuprofen can reduce hypoxia-inducible factors HIF- $1 \alpha$ and HIF-2 $\alpha$ [183]. The state of hypoxia creates a shortage of oxygen free radicals, which is primarily how ionizing radiation damages DNA, so by inhibiting the hypoxic state, there is more of a chance for the cells to be damaged by radiation. At this time, it is unclear why only the astrocytes responded to the radiosensitizing effects of KZ-41.

Treatment with KZ-41 in conjunction with radiation was different than specific antibody treatments with radiation. This indicates that a broad spectrum anti-inflammatory inhibitor may not be the optimum approach as an interventional therapy for radiation damage, and a molecule/paathway specific approach should be considered. In addition, as shown using 
$\mathrm{KZ}-41$, the in vivo and histological methods that have been developed in this work can be used to screen drugs aimed at limiting the acute inflammatory response following cranial radiation.

\subsection{Early markers of radiation-induced damage}

\subsubsection{Gene expression}

Each microarray chip that was analyzed contained 45,101 different genes. By evaluating the changes in expression of these genes we have begun a comprehensive analysis of radiation effects at the very basic level. There is a wealth of information that has been generated and there is much analysis to be carried out, but as can be seen from the genes presented, having this data will allow us to investigate the changes occurring at the mRNA level to better understand where the observed damage is coming from. A better understanding of the initiation of the damage, will enable more focused studies into the presentation of the damage as well as a method to prevent the damage from happening.

Our results show that there is a paracrine response to radiation that is initiated primarily by chemotactic cytokines through the cytokine-cytokine receptor interaction pathway. These are pro-inflammatory chemokines that are known to recruit immune cells to sites of damage. The identification of an inflammatory response at the gene level indicates that there is an acute inflammatory state that is created following a single dose of cranial irradiation, which could be a target for interventional therapy. Our results also allow us to delineate the damage to the vasculature from the parenchymal damage. Since our overall hypothesis is that the vasculature is a major source of damage (early and lateterm), identifying markers of this damage will provide a method for a targeted approach for monitoring vasculature damage following radiation. Our results show that there are genes that are known to be related to neurological/neurodegenerative diseases that become upregulated in the acute phase following radiation. It is possible that the alteration in these genes may be the underlying reason for the cognitive deficits following radiation, and if treated properly, may be the key to limiting these deficits.

A main hypothesis of this work in general is that limiting/eliminating the acute damage will minimize long-term damage following radiation, especially the cognitive damage. One gene that was down-regulated (fold change -2.08) following radiation and also to be related to a neurodegenerative condition is APP. As previously stated, APP (amyloid precursor protein) is known to generate amyloid beta amino acid peptides, a main component of the amyloid plaques found in Alzheimer's disease. There is currently an agent (memantine) being explored to limit the radiation-induced cognitive deficits, a neurodegenerative effect, that has been shown effective in improving cognitive impairment in Alzheimer's patients. Memantine is a non-competitive $\mathrm{N}$-methyl-D-aspartate receptor antagonist, that works by inhibiting the overstimulation of glutamate [185]. In Alzheimer's patients with mild to moderate vascular dementia or moderate to severe Alzheimer's type dementia, memantine has been shown to improve cognition $[186,187,188]$. Other treatments that have been tested for neuronal protection following cranial radiation include lithium and podophyllum hexandrum. If steps can be taken to replace the levels of APP this could potentially be one way to limit cognitive deficits following radiation. 


\subsubsection{Serum cytokines}

Damage to the blood-brain barrier is most likely to trigger a systemic response in the body's efforts to recover. Circulating cytokines could indicate the level of such response and might allow us to elucidate the mechanisms triggered by the localized damage induced by radiation. In our experiments, the serum cytokine GCSF was the only cytokine that showed a significant increase following irradiation treatment localized to the brain. The cytokine GCSF is involved in stimulating the production of and the recruitment of neutrophils, which then migrate to the site of inflammation. GCSF was only elevated at 15-days postRT indicating a late response to irradiation damage. This might indicate that the majority of the irradiation-induced inflammatory response is limited to the immediate vicinity, rather than circulating in the serum. In addition, as shown by Merchant et al [154], cytokine levels can be used to predict the outcome of patients following CNS radiation, and if explored further could provide a way to monitor patients following radiation and give interventional therapies accordingly. 


\section{Chapter 8}

\section{Conclusions and Future Work}

\subsection{Conclusions}

The goal of the current work was to elucidate the role of the inflammatory response in the initiation and propagation of the acute microvasculature changes, following cranial irradiation, by evaluating the role of TNF. This work also investigated a novel anti-inflammatory agent, KZ-41, for use as a potential interventional therapy in preventing the radiationinduced inflammatory response. In addition, this work generated the gene profile of the mouse brain in order to characterize the acute damage following radiation. The main conclusions from this work are the following:

- A single dose of 20-Gy cranial irradiation induces the expression of the inflammatory molecules TNF and ICAM-1 in the brain at 24- and 48-hrs after irradiation.

- The inhibition of TNF or ICAM-1 via antibodies given in conjunction with radiation can abrogate the radiation-induced inflammatory response.

- TNF plays a bigger role in radiation-induced inflammation than ICAM-1, and as such, its inhibition is more potent.

- The anti-inflammatory agent KZ-41 can inhibit radiation-induced vascular effects, but acts as a sensitizer to astrocytes similar to other NSAID's.

- There is a significant increase in astrogliosis following radiation that could persist and be a key factor in long-term damage, and that appears to be related to the inflammatory state in the brain.

- There are early markers of inflammation and damage in the brain occurring at the gene level that may be responsible for the late effects observed following radiation.

- Techniques were established to evaluate mRNA and protein expression that, in conjunction with our in vivo measurements, gives us the tools to begin answering mechanistic questions about what is happening in the brain following cranial irradiation and ways to intervene.

\subsection{Future Work}

Understanding the effects that radiation has on the vasculature of the brain, and the role that the inflammatory response plays is key to limiting late-term radiation damage. The current work investigated the role of TNF and ICAM-1 as the mechanisms thru which radiation-induced inflammation occurs. The modulation of either molecule, via specific an- 
tibodies, inhibited the inflammation-associated radiation-induced vascular responses. A novel anti-inflammatory agent, KZ-41 was investigated as an interventional therapy, but showed that the broad inhibition of the inflammatory response may not be optimal. In addition, gene expression data was generated that will provide more, comprehensive insight to the changes that are occurring immediately following radiation. Although the current studies have identified some mechanisms thru which radiation-induced damage happens, further work needs to be done. Below are some of the potential future studies to continue to understand radiation damage to the brain.

The mechanism of increases in BBB permeability following cranial irradiation is thought to be alterations in the tight junctions and vesicle formation. In previous studies, electron microscopy and histology were used to evaluate the endothelial cells and their response following radiation. The current study showed that inhibiting the expression of TNF or ICAM-1 abrogated the radiation-induced changes in BBB permeability, however the mechanism by which this occurs is unclear. To elucidate the mechanism by which this is happening, one could look at the expression of cytoskeletal proteins before and after irradiation, with and without treatment. One could also evaluate the changes in cytoskeletal protein expression at early time points and late time points, to relate the early vascular changes to late-term changes.

The current work focused on the acute changes to the vasculature in response to radiation, but did not address the late-term changes. There exists a controversy in the field of radiation research about whether a relationship exists between the early effects from radiation and the long-term effects of radiation. We hypothesize that abrogating the early damage will limit the late-term damage, but to test this, one needs to treat during the acute phase, and then evaluate the vascular parameters at the late time points. The current work shows that TNF would be an effective target to evaluate this hypothesis.

$\mathrm{KZ}-41$ is a novel agent that is thought to work thru the NF- $\kappa \mathrm{B}$ pathway, which is known to up-regulate ICAM-1. When KZ-41 was administered, the vascular effects of radiation were inhibited, but the astrocytic response was amplified. The mechanism by which KZ-41 affected the astrocytes is unknown, and is a point of interest for further research. A future study could evaluate the effectiveness of a known NF- $\kappa \mathrm{B}$ inhibitor in comparison to KZ41. This would help elucidate the true effects of inhibiting NF- $\kappa \mathrm{B}$. If the specific inhibitor showed the same response as KZ-41, it could imply that the role NF- $\kappa$ B plays in radiation damage is separate from its activation by the TNF pathway, and is not an effective target for limiting radiation-induced damage.

Microarray analysis was used to evaluate the changes in gene expression at 2-hrs after a single dose of cranial irradiation. This study looked at the significantly altered genes that are involved in the inflammatory, vascular and parenchymal responses, as well as gene related to neurostructural alterations. The genes evaluated are but a small subset of the genes observed to be significantly altered, so further analysis is needed to better understand the changes occurring. In addition, the generated data is from animals with radiation only. Future work could evaluate the gene expression changes in animals that are treated with radiation and TNF or ICAM-1 antibodies. The would allow for the evaluation of the shift in gene expression induced by the inhibition of the acute inflammatory response following radiation, to better understand what role inflammation is playing in radiation-induced damage. In addition, evaluating the alterations in the gene expression in both radiation-only 
and radiation and TNF or ICAM-1 antibody treated groups at late time points could help relate the acute radiation changes to late radiation changes.

The current study administered radiation locally to a strip of brain, with lead body shielding to ensure that other areas did not receive a measurable radiation dose. It is possible to control radiation exposure, but the biological response to radiation may not be limited to the irradiated tissue, especially in the case of cytokines, which are released into the blood stream. This work measured serum cytokine levels at various time points after 20-Gy radiation, but only showed a significant difference in one cytokine (GCSF) at one time point (15-days post-irradiation). It is possible that there were experimental errors (such as the time of day the blood was collected and time between collection and serum extraction) in the collection and analysis of this data, and suggest it be repeated to either confirm these results or generate different results. It is also suggested that other cytokines be considered for analysis.

The animal model used in this work employed a cranial window to visualize the vasculature of the brain. This allows access to the superficial pial vessels of the brain. These vessels are sufficient for evaluating the vasculature parameters we are interested in, as they have been shown to possess the characteristics of the blood-brain barrier, but they cannot provide a complete picture of what is happening in the brain. It would be very beneficial if future studies could employ whole brain imaging technologies to evaluate the effects of radiation on the brain as a whole rather that a small part, and be more clinically relevant.

Understanding the effects of radiation on normal tissue is critical in limiting radiation toxicity during treatment for brain tumors. However, it is known that the presence of a tumor alters the well-controlled microenvironment of the brain, and as such, may effect the way that normal tissue responds to radiation damage. It is essential that future studies include a tumor to mimic the environment in the brain that is created with tumor presence. Any treatment that has the ability to protect the normal tissue, including anti-TNF treatments, must be evaluated with a tumor present to ensure that is does not reduce the efficacy of radiation therapy. 


\section{List of References}

[1] "Report of the brain tumor progress review group," pp. 6-22, 2000, Accessed on: 09/06/2008. Available: http://prg.nci.nih.gov/pdfprgreports/2000braintumor.pdf

[2] M. T. Truong, "Current role of radiation therapy in the managment of malignant brain tumors," Hematol Oncol Clin North Am, vol. 20, pp. 431-453, 2006.

[3] T. J. FitzGerald, J. Aronowitz, M. Giulia Cicchetti, G. Fisher, S. Kadish, Y. C. Lo, C. Mayo, S. McCauley, J. Meyer, R. Pieters, and A. Sherman, "The effect of radiation therapy on normal tissue function," Hematol Oncol Clin North Am, vol. 20, no. 1, pp. 141-63, 2006.

[4] N. D. Doolittle, C. P. Anderson, W. A. Bleyer, J. G. Cairncross, T. Cloughesy, S. L. Eck, P. Guastadisegni, W. A. Hall, L. L. Muldoon, S. J. Patel, D. Peereboom, T. Siegal, and E. A. Neuwelt, "Importance of dose intensity in neuro-oncology clinical trials: summary report of the sixth annual meeting of the blood-brain barrier disruption consortium," Neuro-oncol, vol. 3, no. 1, pp. 46-54, 2001.

[5] "Primary brain tumors in the United States statistical report," Central Brain Tumor Registry of US, Hinsdale, IL, Tech. Rep., 2007.

[6] E. B. Morris, A. Gajjar, J. O. Okuma, Y. Yasui, D. Wallace, L. E. Kun, T. E. Merchant, M. Fouladi, A. Broniscer, L. L. Robison, and M. M. Hudson, "Survival and late mortality in long-term survivors of pediatric cns tumors," J Clin Oncol, vol. 25, no. 12, pp. 1532-8, 2007.

[7] P. J. Tofilon and J. R. Fike, "The radioresponse of the central nervous system: a dynamic process," Radiat Res, vol. 153, no. 4, pp. 357-370, 2000.

[8] T. E. Schultheiss, L. E. Kun, K. K. Ang, and L. C. Stephens, "Radiation response of the central nervous system," Int J Radiat Oncol Biol Phys, vol. 31, no. 5, pp. 10931112, 1995.

[9] H. Yuan, M. W. Gaber, T. McColgan, M. D. Naimark, M. F. Kiani, and T. E. Merchant, "Radiation-induced permeability and leukocyte adhesion in the rat blood- brain barrier: modulation with anti-icam-1 antibodies," Brain Res, vol. 969, no. 1-2, pp. 59-69, 2003.

[10] N. M. Roth, M. R. Sontag, and M. F. Kiani, "Early effects of ionizing radiation on the microvascular networks in normal tissue," Radiat Res, vol. 151, no. 3, pp. 270-277, 1999.

[11] H. B. Stone, C. N. Coleman, M. S. Anscher, and W. H. McBride, "Effects of radiation on normal tissue: consequences and mechanisms," Lancet Oncol, vol. 4, no. 9, pp. 529-536, 2003. 
[12] H. B. Stone, W. H. McBride, and C. N. Coleman, "Modifying normal tissue damage postirradiation. Report of a workshop sponsored by the Radiation Research Program, National Cancer Institute, Bethesda, Maryland, September 6-8, 2000," Radiat Res, vol. 157, no. 2, pp. 204-23, 2002.

[13] J. W. Denham, M. Hauer-Jensen, and L. J. Peters, "Is it time for a new formalism to categorize normal tissue radiation injury?" Int J Radiat Oncol Biol Phys, vol. 50, no. 5, pp. 1105-6, 2001.

[14] H. Kim, J. M. Lee, J. S. Park, S. A. Jo, Y.-O. Kim, C.-W. Kim, and I. Jo, "Dexamethasone coordinately regulates angiopoietin-1 and vegf: a mechanism of glucocorticoidinduced stabilization of blood-brain barrier." Biochem Biophys Res Commun, vol. 372, no. 1, pp. 243-248, 2008.

[15] C. Paul and C. Bolton, "Inhibition of blood-brain barrier disruption in experimental allergic encephalomyelitis by short-term therapy with dexamethasone or cyclosporin a." Int J Immunopharmacol, vol. 17, no. 6, pp. 497-503, 1995.

[16] H. Yuan, D. J. Goetz, M. W. Gaber, A. C. Issekutz, T. E. Merchant, and M. F. Kiani, "Radiation-induced up-regulation of adhesion molecules in brain microvasculature and their modulation by dexamethasone," Radiat Res, vol. 163, no. 5, pp. 544-551, 2005.

[17] E. J. Hall and A. J. Giaccia, Radiobiology for the Radiologist, 6th ed. Philadelphia, PA: Lippincott Williams \& Wilkins, 2006.

[18] J. T. Bushberg, J. A. Seibert, E. M. Leidholdt, and J. M. Boone, The Essential Physics of Medical Imaging, 2nd ed. Philadelphia, PA: Lippincott Williams, \& Wilkins, 2002.

[19] R. K. Hobbie, Intermediate physics for medicine and biology. New York, NY: Springer-Verlag Inc., 1997.

[20] S. Otsuka, J. A. Coderre, P. L. Micca, G. M. Morris, J. W. Hopewell, R. Rola, and J. R. Fike, "Depletion of neural precursor cells after local brain irradiation is due to radiation dose to the parenchyma, not the vasculature," Radiat Res, vol. 165, no. 5, pp. 582-91, 2006.

[21] B. W. Schuller, P. J. Binns, K. J. Riley, L. Ma, M. F. Hawthorne, and J. A. Coderre, "Selective irradiation of the vascular endothelium has no effect on the survival of murine intestinal crypt stem cells," Proc Natl Acad Sci U S A, vol. 103, no. 10, pp. 3787-92, 2006.

[22] N. Lyubimova and J. W. Hopewell, "Experimental evidence to support the hypothesis that damage to vascular endothelium plays the primary role in the development of late radiation-induced cns injury," Br J Radiol, vol. 77, no. 918, pp. 488-92, 2004.

[23] J. A. Coderre, G. M. Morris, P. L. Micca, J. W. Hopewell, I. Verhagen, B. J. Kleiboer, and A. J. van der Kogel, "Late effects of radiation on the central nervous system: role of vascular endothelial damage and glial stem cell survival," Radiat Res, vol. 166, no. 3, pp. 495-503, 2006. 
[24] B. W. Schuller, A. B. Rogers, K. S. Cormier, K. J. Riley, P. J. Binns, R. Julius, M. F. Hawthorne, and J. A. Coderre, "No significant endothelial apoptosis in the radiationinduced gastrointestinal syndrome," Int J Radiat Oncol Biol Phys, vol. 68, no. 1, pp. 205-10, 2007.

[25] A. R. Pries, T. W. Secomb, and P. Gaehtgens, "Design principles of vascular beds." Circ Res, vol. 77, no. 5, pp. 1017-1023, 1995.

[26] J. Panes, D. C. Anderson, M. Miyasaka, and D. N. Granger, "Role of leukocyteendothelial cell adhesion in radiation-induced microvascular dysfunction in rats," Gastroenterology, vol. 108, no. 6, pp. 1761-1769, 1995.

[27] D. J. Acheson, Elementary fluid dynamics. Cary, NC: Oxford Press, 1992.

[28] Y. C. Fung, Biomechanics: circulation. New York, NY: Springer-Verlag Inc., 1999.

[29] C. C. Michel and C. R. Neal, "Openings through endothelial cells associated with increased microvascular permeability," Microcirculation, vol. 6, no. 1, pp. 45-54, 1999.

[30] S. Quarmby, P. Kumar, and S. Kumar, "Radiation-induced normal tissue injury: role of adhesion molecules in leukocyte-endothelial cell interactions," Int J Cancer, vol. 82, no. 3, pp. 385-395, 1999.

[31] M. W. Bradbury, "The blood-brain barrier. transport across the cerebral endothelium," Circ Res, vol. 57, no. 2, pp. 213-222, 1985.

[32] W. H. Oldendorf, M. E. Cornford, and W. J. Brown, "The large apparent work capability of the blood-brain barrier: a study of the mitochondrial content of capillary endothelial cells in brain and other tissues of the rat," Ann Neurol, vol. 1, no. 5, pp. 409-417, 1977.

[33] D. W. Miller, "Immunobiology of the blood-brain barrier," J Neurovirol, vol. 5, no. 6, pp. 570-578, 1999.

[34] K. Kacem, P. Lacombe, J. Seylaz, and G. Bonvento, "Structural organization of the perivascular astrocyte endfeet and their relationship with the endothelial glucose transporter: a confocal microscopy study," Glia, vol. 23, no. 1, pp. 1-10, 1998.

[35] H. K. Kimelberg and M. D. Norenberg, "Astrocytes," Sci Am, vol. 260, no. 4, pp. 66-72, 1989.

[36] W. G. Mayhan, "Regulation of blood-brain barrier permeability," Microcirculation, vol. 8, no. 2, pp. 89-104, 2001.

[37] W. A. Banks, "Physiology and pathology of the blood-brain barrier: implications for microbial pathogenesis, drug delivery and neurodegenerative disorders," J Neurovirol, vol. 5, no. 6, pp. 538-555, 1999.

[38] H. Davson and M. B. Segal, Physiology of the CSF and blood-brain barriers. Boca Raton, FL: CRC Press, 1996.

[39] P. Rubin, D. M. Gash, J. T. Hansen, D. F. Nelson, and J. P. Williams, "Disruption of the blood-brain barrier as the primary effect of cns irradiation," Radiother Oncol, vol. 31, no. 1, pp. 51-60, 1994. 
[40] R. A. Nordal and C. S. Wong, "Molecular targets in radiation-induced blood-brain barrier disruption," Int J Radiat Oncol Biol Phys, vol. 62, no. 1, pp. 279-287, 2005.

[41] M. Johansson, A. T. Bergenheim, A. Widmark, and R. Henriksson, "Effects of radiotherapy and estramustine on the microvasculature in malignant glioma," Br J Cancer, vol. 80, no. 1-2, pp. 142-148, 1999.

[42] R. J. Miller and O. Meucci, "Aids and the brain: is there a chemokine connection?" Trends Neurosci, vol. 22, no. 10, pp. 471-479, 1999.

[43] C. F. Brosnan, Introduction to the Blood-Brain Barrier Methodology, Biology, and Pathology. New York, NY: Cambridge University Press, 1998, ch. Brain microvasculature in multiple sclerosis, pp. 386-400.

[44] H. M. Wisniewski, Introduction to the blood brain barrier methodology, biology and pathology, 1st ed. New York, NY: Cambridge University Press, 1998, ch. Microvascular pathology in cerebrovascular ischemia.

[45] C. K. Petito, Introduction to the Blood-Brain Barrier Methodology, Biology, and Pathology. New York, NY: Cambridge University Press, 1998, ch. HIV infection and the blood-brain barrier, pp. 419-433.

[46] D. J. Stewart, "A critique of the role of the blood-brain barrier in the chemotherapy of human brain tumors," J Neurooncol, vol. 20, no. 2, pp. 121-139, 1994.

[47] H. S. Reinhold, W. Calvo, J. W. Hopewell, and A. P. van der Berg, "Development of blood vessel-related radiation damage in the fimbria of the central nervous system." Int J Radiat Oncol Biol Phys, vol. 18, no. 1, pp. 37-42, 1990.

[48] W. Calvo, J. W. Hopewell, H. S. Reinhold, and T. K. Yeung, "Time- and dose-related changes in the white matter of the rat brain after single doses of x rays," Br J Radiol, vol. 61 , no. 731 , pp. 1043-52, 1988.

[49] C. Belka, W. Budach, R. D. Kortmann, and M. Bamberg, "Radiation induced cns toxicity-molecular and cellular mechanisms," Br J Cancer, vol. 85, no. 9, pp. 12331239, 2001.

[50] F. Paris, Z. Fuks, A. Kang, P. Capodieci, G. Juan, D. Ehleiter, A. Haimovitz-Friedman, C. Cordon-Cardo, and R. Kolesnick, "Endothelial apoptosis as the primary lesion initiating intestinal radiation damage in mice," Science, vol. 293, no. 5528, pp. 293297, 2001.

[51] L. A. Pena, Z. Fuks, and R. N. Kolesnick, "Radiation-induced apoptosis of endothelial cells in the murine central nervous system: protection by fibroblast growth factor and sphingomyelinase deficiency," Cancer Res, vol. 60, no. 2, pp. 321-327, 2000.

[52] S. Quarmby, R. D. Hunter, and S. Kumar, "Irradiation induced expression of cd31, icam-1 and vcam-1 in human microvascular endothelial cells," Anticancer Res, vol. 20, no. 5B, pp. 3375-3381, 2000.

[53] L. F. Fajardo, "Morphologic patterns of radiation injury," Front Radiat Ther Oncol, vol. 23, pp. 75-84, 1989. 
[54] D. Gabrys, O. Greco, G. Patel, K. M. Prise, G. M. Tozer, and C. Kanthou, "Radiation effects on the cytoskeleton of endothelial cells and endothelial monolayer permeability," Int J Radiat Oncol Biol Phys, vol. 69, no. 5, pp. 1553-62, 2007.

[55] W. R. Brown, R. M. Blair, D. M. Moody, C. R. Thore, S. Ahmed, M. E. Robbins, and K. T. Wheeler, "Capillary loss precedes the cognitive impairment induced by fractionated whole-brain irradiation: a potential rat model of vascular dementia," $J$ Neurol Sci, vol. 257, no. 1-2, pp. 67-71, 2007.

[56] C. S. Chiang, J. H. Hong, A. Stalder, J. R. Sun, H. R. Withers, and W. H. McBride, "Delayed molecular responses to brain irradiation," Int J Radiat Biol, vol. 72, no. 1, pp. 45-53, 1997.

[57] M. W. Gaber, O. M. Sabek, K. Fukatsu, H. G. Wilcox, M. F. Kiani, and T. E. Merchant, "Differences in icam-1 and tnf-alpha expression between large single fraction and fractionated irradiation in mouse brain," Int J Radiat Biol, vol. 79, no. 5, pp. 359-366, 2003.

[58] S. Kyrkanides, J. A. Olschowka, J. P. Williams, J. T. Hansen, and M. K. O’Banion, "Tnf alpha and il-1beta mediate intercellular adhesion molecule-1 induction via microgliaastrocyte interaction in cns radiation injury," $J$ Neuroimmunol, vol. 95, no. 1-2, pp. 95-106, 1999.

[59] J. H. Tsai, S. Makonnen, M. Feldman, C. M. Sehgal, A. Maity, and W. M. Lee, "Ionizing radiation inhibits tumor neovascularization by inducing ineffective angiogenesis," Cancer Biol Ther, vol. 4, no. 12, pp. 1395-1400, 2005.

[60] M. W. Dewhirst, R. Richardson, I. Cardenas-Navia, and Y. Cao, "The relationship between the tumor physiologic microenvironment and angiogenesis," Hematol Oncol Clin North Am, vol. 18, no. 5, pp. 973-90, 2004.

[61] R. A. Nordal, A. Nagy, M. Pintilie, and C. S. Wong, "Hypoxia and hypoxia-inducible factor-1 target genes in central nervous system radiation injury: a role for vascular endothelial growth factor," Clin Cancer Res, vol. 10, no. 10, pp. 3342-3353, 2004.

[62] C. L. Limoli, E. Giedzinski, R. Rola, S. Otsuka, T. D. Palmer, and J. R. Fike, "Radiation response of neural precursor cells: linking cellular sensitivity to cell cycle checkpoints, apoptosis and oxidative stress," Radiat Res, vol. 161, no. 1, pp. 17-27, 2004.

[63] J. R. Fike, R. Rola, and C. L. Limoli, "Radiation response of neural precursor cells," Neurosurg Clin N Am, vol. 18, no. 1, pp. 115-27, 2007.

[64] S. Mizumatsu, M. L. Monje, D. R. Morhardt, R. Rola, T. D. Palmer, and J. R. Fike, "Extreme sensitivity of adult neurogenesis to low doses of x-irradiation," Cancer Res, vol. 63, no. 14, pp. 4021-7, 2003.

[65] L. F. Fajardo, "The pathology of ionizing radiation as defined by morphologic patterns," Acta Oncol, vol. 44, no. 1, pp. 13-22, 2005.

[66] J. L. Daigle, J. H. Hong, C. S. Chiang, and W. H. McBride, "The role of tumor necrosis factor signaling pathways in the response of murine brain to irradiation," Cancer Res, vol. 61, no. 24, pp. 8859-8865, 2001. 
[67] G. Di Chiro, E. Oldfield, D. C. Wright, D. De Michele, D. A. Katz, N. J. Patronas, J. L. Doppman, S. M. Larson, M. Ito, and C. V. Kufta, "Cerebral necrosis after radiotherapy and/or intraarterial chemotherapy for brain tumors: Pet and neuropathologic studies," Am J Roentgenol, vol. 150, no. 1, pp. 189-197, 1988.

[68] H. P. Schlemmer, P. Bachert, M. Henze, R. Buslei, K. K. Herfarth, J. Debus, and G. van Kaick, "Differentiation of radiation necrosis from tumor progression using proton magnetic resonance spectroscopy," Neuroradiology, vol. 44, no. 3, pp. 216-222, 2002.

[69] S. Y. Hwang, J. S. Jung, T. H. Kim, S. J. Lim, E. S. Oh, J. Y. Kim, K. A. Ji, E. H. Joe, K. H. Cho, and I. O. Han, "Ionizing radiation induces astrocyte gliosis through microglia activation," Neurobiol Dis, vol. 21, no. 3, pp. 457-67, 2006.

[70] H. Yuan, M. W. Gaber, K. Boyd, C. M. Wilson, M. F. Kiani, and T. E. Merchant, "Effects of fractionated radiation on the brain vasculature in a murine model: bloodbrain barrier permeability, astrocyte proliferation, and ultrastructural changes," Int $J$ Radiat Oncol Biol Phys, vol. 66, no. 3, pp. 860-6, 2006.

[71] P. L. Debbage, S. Seidl, A. Kreczy, P. Hutzler, M. Pavelka, and P. Lukas, "Vascular permeability and hyperpermeability in a murine adenocarcinoma after fractionated radiotherapy: an ultrastructural tracer study," Histochem Cell Biol, vol. 114, no. 4, pp. 259-275, 2000.

[72] D. d'Avella, R. Cicciarello, F. Albiero, M. Mesiti, M. E. Gagliardi, E. Russi, A. d'Aquino, F. Tomasello, and S. d'Aquino, "Quantitative study of blood-brain barrier permeability changes after experimental whole-brain radiation." Neurosurgery, vol. 30, no. 1, pp. 30-34, 1992.

[73] G. Eissner, F. Kohlhuber, M. Grell, M. Ueffing, P. Scheurich, A. Hieke, G. Multhoff, G. W. Bornkamm, and E. Holler, "Critical involvement of transmembrane tumor necrosis factor-alpha in endothelial programmed cell death mediated by ionizing radiation and bacterial endotoxin," Blood, vol. 86, no. 11, pp. 4184-4193, 1995.

[74] C. Michiels, “Endothelial cell functions," J Cell Physiol, vol. 196, no. 3, pp. 430-443, 2003.

[75] C. D. Buckley, R. G. Ed, G. B. Nash, and K. Raza, "Endothelial cells, fibroblasts and vasculitis," Rheumatology.(Oxford), vol. 44, no. 7, pp. 860-863, 2005.

[76] D. E. Hallahan, J. Kuchibhotla, and C. Wyble, "Cell adhesion molecules mediate radiation-induced leukocyte adhesion to the vascular endothelium," Cancer Res, vol. 56, no. 22, pp. 5150-5155, 1996.

[77] D. E. Hallahan and S. Virudachalam, "Accumulation of p-selectin in the lumen of irradiated blood vessels," Radiat Res, vol. 152, no. 1, pp. 6-13, 1999.

[78] H. Kimura, N. Z. Wu, R. Dodge, D. P. Spencer, B. M. Klitzman, T. M. Mclntyre, and M. W. Dewhirst, "Inhibition of radiation-induced up-regulation of leukocyte adhesion to endothelial cells with the platelet-activating factor inhibitor, bn52021," Int J Radiat Oncol Biol Phys, vol. 33, no. 3, pp. 627-33, 1995. 
[79] J. Panes and D. N. Granger, "Leukocyte-endothelial cell interactions: molecular mechanisms and implications in gastrointestinal disease," Gastroenterology, vol. 114, no. 5, pp. 1066-1090, 1998.

[80] J. Panes, M. Perry, and D. N. Granger, "Leukocyte-endothelial cell adhesion: avenues for therapeutic intervention," Br J Pharmacol, vol. 126, no. 3, pp. 537-50, 1999.

[81] G. R. J. Gordon, S. J. Mulligan, and B. A. MacVicar, "Astrocyte control of the cerebrovasculature." Glia, vol. 55, no. 12, pp. 1214-1221, 2007.

[82] D. Fukumura, F. Yuan, M. Endo, and R. K. Jain, "Role of nitric oxide in tumor microcirculation. blood flow, vascular permeability, and leukocyte-endothelial interactions," Am J Pathol, vol. 150, no. 2, pp. 713-725, 1997.

[83] J. P. Muizelaar, G. J. Bouma, J. E. Levasseur, and H. A. Kontos, "Effect of hematocrit variations on cerebral blood flow and basilar artery diameter in vivo," Am J Physiol, vol. 262, no. 4 Pt 2, pp. H949-H954, 1992.

[84] D. G. Baker and R. J. Krochak, "The response of the microvascular system to radiation: a review," Cancer Invest, vol. 7, no. 3, pp. 287-94, 1989.

[85] K. R. Stenmark, K. A. Fagan, and M. G. Frid, "Hypoxia-induced pulmonary vascular remodeling: cellular and molecular mechanisms," Circ Res, vol. 99, no. 7, pp. 67591, 2006.

[86] S. Ogawa, M. Clauss, K. Kuwabara, R. Shreeniwas, C. Butura, S. Koga, and D. Stern, "Hypoxia induces endothelial cell synthesis of membrane-associated proteins," Proc Natl Acad Sci U S A, vol. 88, no. 21, pp. 9897-901, 1991.

[87] S. Fischer, M. Wobben, H. H. Marti, D. Renz, and W. Schaper, "Hypoxia-induced hyperpermeability in brain microvessel endothelial cells involves vegf-mediated changes in the expression of zonula occludens-1," Microvasc Res, vol. 63, no. 1, pp. 70-80, 2002.

[88] A. Hunter, A. Hendrikse, M. Renan, and R. Abratt, "Does the tumor microenvironment influence radiation-induced apoptosis?" Apoptosis, vol. 11, no. 10, pp. 172735, 2006.

[89] M. S. Anscher, L. Chen, Z. Rabbani, S. Kang, N. Larrier, H. Huang, T. V. Samulski, M. W. Dewhirst, D. M. Brizel, R. J. Folz, and Z. Vujaskovic, "Recent progress in defining mechanisms and potential targets for prevention of normal tissue injury after radiation therapy," Int J Radiat Oncol Biol Phys, vol. 62, no. 1, pp. 255-259, 2005.

[90] M. Hockel and P. Vaupel, "Tumor hypoxia: definitions and current clinical, biologic, and molecular aspects," J Natl Cancer Inst, vol. 93, no. 4, pp. 266-76, 2001.

[91] M. Pekny and M. Pekna, "Astrocyte intermediate filaments in cns pathologies and regeneration," J Pathol, vol. 204, no. 4, pp. 428-37, 2004.

[92] M. Nedergaard, B. Ransom, and S. A. Goldman, "New roles for astrocytes: redefining the functional architecture of the brain," Trends Neurosci, vol. 26, no. 10, pp. 523-30, 2003. 
[93] J. Schummers, H. Yu, and M. Sur, "Tuned responses of astrocytes and their influence on hemodynamic signals in the visual cortex," Science, vol. 320, no. 5883, pp. 163843, 2008.

[94] B. Ransom, T. Behar, and M. Nedergaard, "New roles for astrocytes (stars at last)," Trends Neurosci, vol. 26, no. 10, pp. 520-2, 2003.

[95] A. Bonni, Y. Sun, M. Nadal-Vicens, A. Bhatt, D. A. Frank, I. Rozovsky, N. Stahl, G. D. Yancopoulos, and M. E. Greenberg, "Regulation of gliogenesis in the central nervous system by the jak-stat signaling pathway," Science, vol. 278, no. 5337, pp. 477-83, 1997.

[96] N. J. Abbott, L. Ronnback, and E. Hansson, "Astrocyte-endothelial interactions at the blood-brain barrier," Nat Rev Neurosci, vol. 7, no. 1, pp. 41-53, 2006.

[97] S. H. Kim, D. J. Lim, Y. G. Chung, T. H. Cho, S. J. Lim, W. J. Kim, and J. K. Suh, "Expression of tnf-alpha and tgf-beta 1 in the rat brain after a single high-dose irradiation," J Korean Med Sci, vol. 17, no. 2, pp. 242-248, 2002.

[98] G. I. Botchkina, M. E. I. I. I. Meistrell, I. L. Botchkina, and K. J. Tracey, "Expression of tnf and tnf receptors (p55 and p75) in the rat brain after focal cerebral ischemia," Mol Med, vol. 3, no. 11, pp. 765-781, 1997.

[99] J. F. Lubar, "Neocortical dynamics: implications for understanding the role of neurofeedback and related techniques for the enhancement of attention," Appl Psychophysiol Biofeedback, vol. 22, no. 2, pp. 111-26, 1997.

[100] S. Atkinson, Y. Q. Li, and C. S. Wong, "Changes in oligodendrocytes and myelin gene expression after radiation in the rodent spinal cord," Int $J$ Radiat Oncol Biol Phys, vol. 57, no. 4, pp. 1093-100, 2003.

[101] C. S. Chiang, W. H. McBride, and H. R. Withers, "Myelin-associated changes in mouse brain following irradiation," Radiother Oncol, vol. 27, no. 3, pp. 229-236, 1993.

[102] C. S. Chiang, K. A. Mason, H. R. Withers, and W. H. McBride, "Alteration in myelinassociated proteins following spinal cord irradiation in guinea pigs," Int $J$ Radiat Oncol Biol Phys, vol. 24, no. 5, pp. 929-37, 1992.

[103] F. L. Mastaglia, W. I. McDonald, J. V. Watson, and K. Yogendran, "Effects of Xradiation on the spinal cord: an experimental study of the morphological changes in central nerve fibres," Brain, vol. 99, no. 1, pp. 101-22, 1976.

[104] P. A. Felts and K. J. Smith, "Blood-brain barrier permeability in astrocyte-free regions of the central nervous system remyelinated by schwann cells," Neuroscience, vol. 75, no. 2, pp. 643-55, 1996.

[105] G. Panagiotakos, G. Alshamy, B. Chan, R. Abrams, E. Greenberg, A. Saxena, M. Bradbury, M. Edgar, P. Gutin, and V. Tabar, "Long-term impact of radiation on the stem cell and oligodendrocyte precursors in the brain," PLOS ONE, vol. 2, no. 7, p. e588, 2007. 
[106] M. W. Epperly, C. A. Sikora, S. J. DeFilippi, J. E. Gretton, D. Bar-Sagi, H. Archer, T. Carlos, H. Guo, and J. S. Greenberger, "Pulmonary irradiation-induced expression of vcam-i and icam-i is decreased by manganese superoxide dismutaseplasmid/liposome (mnsod-pl) gene therapy," Biol Blood Marrow Transplant, vol. 8, no. 4, pp. 175-187, 2002.

[107] G. Hildebrandt, L. Maggiorella, F. Rodel, V. Rodel, D. Willis, and K. R. Trott, "Mononuclear cell adhesion and cell adhesion molecule liberation after x-irradiation of activated endothelial cells in vitro," Int J Radiat Biol, vol. 78, no. 4, pp. 315-325, 2002.

[108] F. Roedel, N. Kley, H. U. Beuscher, G. Hildebrandt, L. Keilholz, P. Kern, R. Voll, M. Herrmann, and R. Sauer, "Anti-inflammatory effect of low-dose x-irradiation and the involvement of a tgf-beta1-induced down-regulation of leukocyte/endothelial cell adhesion," Int J Radiat Biol, vol. 78, no. 8, pp. 711-719, 2002.

[109] S. A. van de and P. T. van der Saag, "Intercellular adhesion molecule-1," J Mol Med, vol. 74, no. 1, pp. 13-33, 1996.

[110] T. Imaizumi, H. Itaya, K. Fujita, D. Kudoh, S. Kudoh, K. Mori, K. Fujimoto, T. Matsumiya, H. Yoshida, and K. Satoh, "Expression of tumor necrosis factor-alpha in cultured human endothelial cells stimulated with lipopolysaccharide or interleukin1alpha," Arterioscler Thromb Vasc Biol, vol. 20, no. 2, pp. 410-415, 2000.

[111] M. E. I. I. I. Meistrell, G. I. Botchkina, H. Wang, E. Di Santo, K. M. Cockroft, O. Bloom, J. M. Vishnubhakat, P. Ghezzi, and K. J. Tracey, "Tumor necrosis factor is a brain damaging cytokine in cerebral ischemia," Shock, vol. 8, no. 5, pp. 341-348, 1997.

[112] J. Friedl, M. Puhlmann, D. L. Bartlett, S. K. Libutti, E. N. Turner, M. F. Gnant, and H. R. Alexander, "Induction of permeability across endothelial cell monolayers by tumor necrosis factor (tnf) occurs via a tissue factor-dependent mechanism: relationship between the procoagulant and permeability effects of tnf," Blood, vol. 100, no. 4, pp. 1334-9, 2002.

[113] F. Bazzoni and B. Beutler, "The tumor necrosis factor ligand and receptor families," N Engl J Med, vol. 334, no. 26, pp. 1717-1725, 1996.

[114] P. R. Clark, T. D. Manes, J. S. Pober, and M. S. Kluger, "Increased icam-1 expression causes endothelial cell leakiness, cytoskeletal reorganization and junctional alterations," J Invest Dermatol, vol. 127, no. 4, pp. 762-74, 2007.

[115] J. K. Min, Y. M. Kim, S. W. Kim, M. C. Kwon, Y. Y. Kong, I. K. Hwang, M. H. Won, J. Rho, and Y. G. Kwon, "Tnf-related activation-induced cytokine enhances leukocyte adhesiveness: induction of icam-1 and vcam-1 via tnf receptor-associated factor and protein kinase c-dependent nf-kappab activation in endothelial cells," J Immunol, vol. 175, no. 1, pp. 531-40, 2005.

[116] N. A. Essani, G. M. McGuire, A. M. Manning, and H. Jaeschke, "Endotoxin-induced activation of the nuclear transcription factor kappa $b$ and expression of e-selectin messenger rna in hepatocytes, kupffer cells, and endothelial cells in vivo," J Immunol, vol. 156, no. 8, pp. 2956-63, 1996. 
[117] Y. Sheng, C. Akesson, K. Holmgren, C. Bryngelsson, V. Giamapa, and R. W. Pero, "An active ingredient of cat's claw water extracts identification and efficacy of quinic acid," J Ethnopharmacol, vol. 96, no. 3, pp. 577-84, 2005.

[118] C. Akesson, H. Lindgren, R. W. Pero, T. Leanderson, and F. Ivars, "Quinic acid is a biologically active component of the uncaria tomentosa extract c-med 100." Int Immunopharmacol, vol. 5, no. 1, pp. 219-229, 2005.

[119] I. Lemaire, V. Assinewe, P. Cano, D. V. Awang, and J. T. Arnason, "Stimulation of interleukin-1 and -6 production in alveolar macrophages by the neotropical liana, uncaria tomentosa (una de gato)," J Ethnopharmacol, vol. 64, no. 2, pp. 109-15, 1999.

[120] M. Wurm, L. Kacani, G. Laus, K. Keplinger, and M. P. Dierich, "Pentacyclic oxindole alkaloids from uncaria tomentosa induce human endothelial cells to release a lymphocyte-proliferation-regulating factor," Planta Med, vol. 64, no. 8, pp. 701-4, 1998.

[121] R. Rizzi, F. Re, A. Bianchi, V. De Feo, F. de Simone, L. Bianchi, and L. A. Stivala, "Mutagenic and antimutagenic activities of uncaria tomentosa and its extracts," $J$ Ethnopharmacol, vol. 38, no. 1, pp. 63-77, 1993.

[122] R. Aquino, V. De Feo, F. De Simone, C. Pizza, and G. Cirino, "Plant metabolites. new compounds and anti-inflammatory activity of uncaria tomentosa," J Nat Prod, vol. 54, no. 2, pp. 453-9, 1991.

[123] R. Aquino, F. De Simone, C. Pizza, C. Conti, and M. L. Stein, "Plant metabolites. structure and in vitro antiviral activity of quinovic acid glycosides from uncaria tomentosa and guettarda platypoda," J Nat Prod, vol. 52, no. 4, pp. 679-85, 1989.

[124] S. Lamm, Y. Sheng, and R. W. Pero, "Persistent response to pneumococcal vaccine in individuals supplemented with a novel water soluble extract of uncaria tomentosa, c-med-100," Phytomedicine, vol. 8, no. 4, pp. 267-74, 2001.

[125] Y. Sheng, L. Li, K. Holmgren, and R. W. Pero, "Dna repair enhancement of aqueous extracts of uncaria tomentosa in a human volunteer study," Phytomedicine, vol. 8, no. 4, pp. 275-82, 2001.

[126] Y. Sheng, C. Bryngelsson, and R. W. Pero, "Enhanced dna repair, immune function and reduced toxicity of c-med-100, a novel aqueous extract from uncaria tomentosa," J Ethnopharmacol, vol. 69, no. 2, pp. 115-26, 2000.

[127] A. S. Mahmoud-Ahmed, S. Atkinson, and C. S. Wong, "Early gene expression profile in mouse brain after exposure to ionizing radiation," Radiat Res, vol. 165, no. 2, pp. 142-54, 2006.

[128] M. W. Gaber, H. Yuan, J. T. Killmar, M. D. Naimark, M. F. Kiani, and T. E. Merchant, "An intravital microscopy study of radiation-induced changes in permeability and leukocyte-endothelial cell interactions in the microvessels of the rat pia mater and cremaster muscle," Brain Res Brain Res Protoc, vol. 13, no. 1, pp. 1-10, 2004. 
[129] N. Z. Wu, B. Klitzman, G. Rosner, D. Needham, and M. W. Dewhirst, "Measurement of material extravasation in microvascular networks using fluorescence videomicroscopy," Microvasc Res, vol. 46, no. 2, pp. 231-253, 1993.

[130] F. Yuan, M. Leunig, D. A. Berk, and R. K. Jain, "Microvascular permeability of albumin, vascular surface area, and vascular volume measured in human adenocarcinoma Is174t using dorsal chamber in scid mice," Microvasc Res, vol. 45, no. 3, pp. 269-289, 1993.

[131] S. D. House and H. H. Lipowsky, "Microvascular hematocrit and red cell flux in rat cremaster muscle," Am J Physiol, vol. 252, no. 1 Pt 2, pp. H211-H222, 1987.

[132] Y. Hong, "Effects of ionizing radiation on normal cerebral microvasculature," Ph.D. dissertation, University of Tennessee Health Science Center, 2003.

[133] H. Baatz, M. Steinbauer, A. G. Harris, and F. Krombach, "Kinetics of white blood cell staining by intravascular administration of rhodamine 6g," Int $J$ Microcirc Clin Exp, vol. 15, no. 2, pp. 85-91, 1995.

[134] B. Zhang, S. Kirov, and J. Snoddy, "Webgestalt: an integrated system for exploring gene sets in various biological contexts." Nucleic Acids Res, vol. 33, no. Web Server issue, pp. W741-8, 2005 Jul 1.

[135] G. Q. Jia, J. A. Gonzalo, C. Lloyd, L. Kremer, L. Lu, C. Martinez-A, B. K. Wershil, and J. C. Gutierrez-Ramos, "Distinct expression and function of the novel mouse chemokine monocyte chemotactic protein-5 in lung allergic inflammation." $J$ Exp Med, vol. 184, no. 5, pp. 1939-1951, 1996.

[136] H.-H. Tsai, E. Frost, V. To, S. Robinson, C. Ffrench-Constant, R. Geertman, R. M. Ransohoff, and R. H. Miller, "The chemokine receptor cxcr2 controls positioning of oligodendrocyte precursors in developing spinal cord by arresting their migration." Cell, vol. 110, no. 3, pp. 373-383, 2002.

[137] F. Bard, C. Cannon, R. Barbour, R. L. Burke, D. Games, H. Grajeda, T. Guido, K. Hu, J. Huang, K. Johnson-Wood, K. Khan, D. Kholodenko, M. Lee, I. Lieberburg, R. Motter, M. Nguyen, F. Soriano, N. Vasquez, K. Weiss, B. Welch, P. Seubert, D. Schenk, and T. Yednock, "Peripherally administered antibodies against amyloid beta-peptide enter the central nervous system and reduce pathology in a mouse model of alzheimer disease," Nat Med, vol. 6, no. 8, pp. 916-919, 2000.

[138] M. P. Mattson, S. W. Barger, K. Furukawa, A. J. Bruce, T. Wyss-Coray, R. J. Mark, and L. Mucke, "Cellular signaling roles of tgf beta, tnf alpha and beta app in brain injury responses and alzheimer's disease," Brain Res Brain Res Rev, vol. 23, no. 1-2, pp. 47-61, 1997.

[139] P. L. McGeer and E. G. McGeer, "Anti-inflammatory drugs in the fight against alzheimer's disease," Ann N Y Acad Sci, vol. 777, pp. 213-220, 1996.

[140] C. Scali, C. Prosperi, M. G. Vannucchi, G. Pepeu, and F. Casamenti, "Brain inflammatory reaction in an animal model of neuronal degeneration and its modulation by an anti-inflammatory drug: implication in alzheimer's disease," Eur J Neurosci, vol. 12, no. 6, pp. 1900-1912, 2000. 
[141] F. Zipp and O. Aktas, "The brain as a target of inflammation: common pathways link inflammatory and neurodegenerative diseases," Trends Neurosci, vol. 29, no. 9, pp. 518-27, 2006.

[142] D. M. Aebersold, P. Burri, K. T. Beer, J. Laissue, V. Djonov, R. H. Greiner, and G. L. Semenza, "Expression of hypoxia-inducible factor-1alpha: a novel predictive and prognostic parameter in the radiotherapy of oropharyngeal cancer," Cancer Res, vol. 61, no. 7, pp. 2911-2916, 2001.

[143] C. G. Lee, M. Heijn, E. di Tomaso, G. Griffon-Etienne, M. Ancukiewicz, C. Koike, K. R. Park, N. Ferrara, R. K. Jain, H. D. Suit, and Y. Boucher, "Anti-vascular endothelial growth factor treatment augments tumor radiation response under normoxic or hypoxic conditions," Cancer Res, vol. 60, no. 19, pp. 5565-70, 2000.

[144] Y. Q. Li, J. R. Ballinger, R. A. Nordal, Z. F. Su, and C. S. Wong, "Hypoxia in radiationinduced blood-spinal cord barrier breakdown," Cancer Res, vol. 61, no. 8, pp. 33483354, 2001.

[145] M. Garcia-Barros, F. Paris, C. Cordon-Cardo, D. Lyden, S. Rafii, A. HaimovitzFriedman, Z. Fuks, and R. Kolesnick, "Tumor response to radiotherapy regulated by endothelial cell apoptosis," Science, vol. 300, no. 5622, pp. 1155-9, 2003.

[146] B. A. Rupnow, A. D. Murtha, R. M. Alarcon, A. J. Giaccia, and S. J. Knox, "Direct evidence that apoptosis enhances tumor responses to fractionated radiotherapy," Cancer Res, vol. 58, no. 9, pp. 1779-1784, 1998.

[147] K. H. Herzog, M. J. Chong, M. Kapsetaki, J. I. Morgan, and P. J. McKinnon, "Requirement for atm in ionizing radiation-induced cell death in the developing central nervous system," Science, vol. 280, no. 5366, pp. 1089-1091, 1998.

[148] M. B. Kastan, D. S. Lim, S. T. Kim, and D. Yang, "Atm-a key determinant of multiple cellular responses to irradiation," Acta Oncol, vol. 40, no. 6, pp. 686-688, 2001.

[149] S. E. Morgan and M. B. Kastan, "p53 and atm: cell cycle, cell death, and cancer," Adv Cancer Res, vol. 71, pp. 1-25, 1997.

[150] E. M. Rosen, S. Fan, I. D. Goldberg, and S. Rockwell, "Biological basis of radiation sensitivity. part 2: Cellular and molecular determinants of radiosensitivity," Oncology (Huntingt), vol. 14, no. 5, pp. 741-757, 2000.

[151] V. Lemaire, M. Koehl, M. Le Moal, and D. N. Abrous, "Prenatal stress produces learning deficits associated with an inhibition of neurogenesis in the hippocampus," Proc Natl Acad Sci U S A, vol. 97, no. 20, pp. 11032-7, 2000.

[152] M. L. Monje and T. Palmer, "Radiation injury and neurogenesis," Curr Opin Neurol, vol. 16, no. 2, pp. 129-34, 2003.

[153] H. van Praag, A. F. Schinder, B. R. Christie, N. Toni, T. D. Palmer, and F. H. Gage, "Functional neurogenesis in the adult hippocampus," Nature, vol. 415, no. 6875, pp. 1030-4, 2002. 
[154] T. Merchant, C. Li, X. Xiong, and M. Gaber, "Cytokine and growth factor responses after radiotherapy for localized ependymoma." Int $J$ Radiat Oncol Biol Phys, 2008 in print.

[155] R. Ansari, M. W. Gaber, B. Wang, C. B. Patillo, C. Miyamoto, and M. F. Kiani, "Antitnfa (tnf-a) treatment abrogates radiation-induced changes in vascular density and tissue oxygenation," Radiat Res, vol. 167, pp. 80-86, 2007.

[156] V. Nguyen, M. W. Gaber, M. R. Sontag, and M. F. Kiani, "Late effects of ionizing radiation on the microvascular networks in normal tissue," Radiat Res, vol. 154, no. 5, pp. 531-536, 2000.

[157] J. Carvalho-Tavares, M. J. Hickey, J. Hutchison, J. Michaud, I. T. Sutcliffe, and P. Kubes, "A role for platelets and endothelial selectins in tumor necrosis factoralpha-induced leukocyte recruitment in the brain microvasculature," Circ Res, vol. 87, no. 12, pp. 1141-8, 2000.

[158] J. L. Wright and R. E. Merchant, "Histopathological effects of intracerebral injections of human recombinant tumor necrosis factor-alpha in the rat," Acta Neuropathol (Berl), vol. 85, no. 1, pp. 93-100, 1992.

[159] J. C. Acker, L. B. Marks, D. P. Spencer, W. Yang, M. A. Avery, R. K. Dodge, G. L. Rosner, and M. W. Dewhirst, "Serial in vivo observations of cerebral vasculature after treatment with a large single fraction of radiation," Radiat Res, vol. 149, no. 4, pp. 350-359, 1998.

[160] E. J. Kunkel, U. Jung, and K. Ley, "Tnf-alpha induces selectin-mediated leukocyte rolling in mouse cremaster muscle arterioles," Am J Physiol, vol. 272, no. 3 Pt 2, pp. H1391-400, 1997.

[161] H. Thorlacius, L. Lindbom, and J. Raud, "Cytokine-induced leukocyte rolling in mouse cremaster muscle arterioles in p-selectin dependent," Am J Physiol, vol. 272, no. 4 Pt 2, pp. H1725-9, 1997.

[162] W. G. Mayhan, "Cellular mechanisms by which tumor necrosis factor-alpha produces disruption of the blood-brain barrier," Brain Res, vol. 927, no. 2, pp. 144-152, 2002.

[163] C. S. Chiang, W. H. McBride, and H. R. Withers, "Radiation-induced astrocytic and microglial responses in mouse brain," Radiother Oncol, vol. 29, no. 1, pp. 60-68, 1993.

[164] A. Larsson, U. Wilhelmsson, M. Pekna, and M. Pekny, "Increased cell proliferation and neurogenesis in the hippocampal dentate gyrus of old gfap(-/-)vim(-/-) mice," Neurochem Res, vol. 29, no. 11, pp. 2069-73, 2004.

[165] U. Wilhelmsson, C. Eliasson, R. Bjerkvig, and M. Pekny, "Loss of gfap expression in high-grade astrocytomas does not contribute to tumor development or progression," Oncogene, vol. 22, no. 22, pp. 3407-11, 2003.

[166] V. Balasingam, T. Tejada-Berges, E. Wright, R. Bouckova, and V. W. Yong, "Reactive astrogliosis in the neonatal mouse brain and its modulation by cytokines," J Neurosci, vol. 14, no. 2, pp. 846-856, 1994. 
[167] K. W. Selmaj, M. Farooq, W. T. Norton, C. S. Raine, and C. F. Brosnan, "Proliferation of astrocytes in vitro in response to cytokines. a primary role for tumor necrosis factor," J Immunol, vol. 144, no. 1, pp. 129-35, 1990.

[168] J. H. Hong, C. S. Chiang, I. L. Campbell, J. R. Sun, H. R. Withers, and W. H. McBride, "Induction of acute phase gene expression by brain irradiation," Int $J$ Radiat Oncol Biol Phys, vol. 33, no. 3, pp. 619-626, 1995.

[169] A. Del Maschio, A. Zanetti, M. Corada, Y. Rival, L. Ruco, M. G. Lampugnani, and E. Dejana, "Polymorphonuclear leukocyte adhesion triggers the disorganization of endothelial cell-to-cell adherens junctions," J Cell Biol, vol. 135, no. 2, pp. 497-510, 1996.

[170] S. Etienne, P. Adamson, J. Greenwood, A. D. Strosberg, S. Cazaubon, and P. O. Couraud, "Icam-1 signaling pathways associated with rho activation in microvascular brain endothelial cells,” J Immunol, vol. 161, no. 10, pp. 5755-5761, 1998.

[171] D. Tweedie, K. Sambamurti, and N. H. Greig, "Tnf-alpha inhibition as a treatment strategy for neurodegenerative disorders: new drug candidates and targets," Curr Alzheimer Res, vol. 4, no. 4, pp. 378-85, 2007.

[172] P. E. Lipsky, D. M. van der Heijde, E. W. St Clair, D. E. Furst, F. C. Breedveld, J. R. Kalden, J. S. Smolen, M. Weisman, P. Emery, M. Feldmann, G. R. Harriman, and R. N. Maini, "Infliximab and methotrexate in the treatment of rheumatoid arthritis. anti-tumor necrosis factor trial in rheumatoid arthritis with concomitant therapy study group," N Engl J Med, vol. 343, no. 22, pp. 1594-602, 2000.

[173] M. L. Harrison, E. Obermueller, N. R. Maisey, S. Hoare, K. Edmonds, N. F. Li, D. Chao, K. Hall, C. Lee, E. Timotheadou, K. Charles, R. Ahern, D. M. King, T. Eisen, R. Corringham, M. DeWitte, F. Balkwill, and M. Gore, "Tumor necrosis factor alpha as a new target for renal cell carcinoma: two sequential phase ii trials of infliximab at standard and high dose," J Clin Oncol, vol. 25, no. 29, pp. 4542-9, 2007.

[174] S. V. Rajkumar, S. R. Hayman, M. Q. Lacy, A. Dispenzieri, S. M. Geyer, B. Kabat, S. R. Zeldenrust, S. Kumar, P. R. Greipp, R. Fonseca, J. A. Lust, S. J. Russell, R. A. Kyle, T. E. Witzig, and M. A. Gertz, "Combination therapy with lenalidomide plus dexamethasone (rev/dex) for newly diagnosed myeloma," Blood, vol. 106, no. 13, pp. 4050-3, 2005.

[175] P. J. Hayden, C. S. Mitsiades, K. C. Anderson, and P. G. Richardson, "Novel therapies in myeloma," Curr Opin Hematol, vol. 14, no. 6, pp. 609-15, 2007.

[176] P. Richardson, T. Hideshima, and K. Anderson, "Thalidomide: emerging role in cancer medicine," Annu Rev Med, vol. 53, pp. 629-57, 2002.

[177] B. E. Turk, H. Jiang, and J. O. Liu, "Binding of thalidomide to alpha1-acid glycoprotein may be involved in its inhibition of tumor necrosis factor alpha production," Proc Natl Acad Sci U S A, vol. 93, no. 15, pp. 7552-6, 1996.

[178] M. D. Groves, V. K. Puduvalli, S. M. Chang, C. A. Conrad, M. R. Gilbert, I. W. Tremont-Lukats, T. J. Liu, P. Peterson, D. Schiff, T. F. Cloughesy, P. Y. Wen, H. Greenberg, L. E. Abrey, L. M. DeAngelis, K. R. Hess, K. R. Lamborn, M. D. Prados, and 
W. K. Yung, "A north american brain tumor consortium (nabtc 99-04) phase ii trial of temozolomide plus thalidomide for recurrent glioblastoma multiforme," $J$ Neurooncol, vol. 81, no. 3, pp. 271-7, 2007.

[179] J. Hirsh, "Risk of thrombosis with lenalidomide and its prevention with aspirin," Chest, vol. 131, no. 1, pp. 275-7, 2007.

[180] N. H. Greig, M. P. Mattson, T. Perry, S. L. Chan, T. Giordano, K. Sambamurti, J. T. Rogers, H. Ovadia, and D. K. Lahiri, "New therapeutic strategies and drug candidates for neurodegenerative diseases: p53 and tnf-alpha inhibitors, and glp-1 receptor agonists," Ann N Y Acad Sci, vol. 1035, pp. 290-315, 2004.

[181] M. Kiaei, S. Petri, K. Kipiani, G. Gardian, D. K. Choi, J. Chen, N. Y. Calingasan, P. Schafer, G. W. Muller, C. Stewart, K. Hensley, and M. F. Beal, "Thalidomide and lenalidomide extend survival in a transgenic mouse model of amyotrophic lateral sclerosis," J Neurosci, vol. 26, no. 9, pp. 2467-73, 2006.

[182] S. T. Palayoor, M. A. Burgos, A. Shoaibi, P. J. Tofilon, and C. N. Coleman, "Effect of radiation and ibuprofen on normoxic renal carcinoma cells overexpressing hypoxiainducible factors by loss of von hippel-lindau tumor suppressor gene function." Clin Cancer Res, vol. 10, no. 12 Pt 1, pp. 4158-4164, 2004.

[183] S. T. Palayoor, P. J. Tofilon, and C. N. Coleman, "Ibuprofen-mediated reduction of hypoxia-inducible factors hif-1alpha and hif-2alpha in prostate cancer cells." Clin Cancer Res, vol. 9, no. 8, pp. 3150-3157, 2003.

[184] S. T. Palayoor, E. A. Bump, S. K. Calderwood, S. Bartol, and C. N. Coleman, "Combined antitumor effect of radiation and ibuprofen in human prostate carcinoma cells." Clin Cancer Res, vol. 4, no. 3, pp. 763-771, 1998.

[185] P. N. Shevtsov, E. F. Shevtsova, and G. S. Burbaeva, "Effect of tacrine, amiridine, akatinol memantine, and triazolam on phosphorylation, structure, and assembly of microtubules from brain microtubular proteins in alzheimer diseases." Bull Exp Biol Med, vol. 145, no. 2, pp. 218-222, 2008.

[186] J. L. Cummings, J. Mackell, and D. Kaufer, "Behavioral effects of current alzheimer's disease treatments: a descriptive review." Alzheimers Dement, vol. 4, no. 1, pp. 4960, 2008.

[187] M. Emre, P. Mecocci, and K. Stender, "Pooled analyses on cognitive effects of memantine in patients with moderate to severe alzheimer's disease." J Alzheimers Dis, vol. 14, no. 2, pp. 193-199, 2008.

[188] D. B. Hogan, P. Bailey, S. Black, A. Carswell, H. Chertkow, B. Clarke, C. Cohen, J. D. Fisk, D. Forbes, M. Man-Son-Hing, K. Lanctot, D. Morgan, and L. Thorpe, "Diagnosis and treatment of dementia: 5. nonpharmacologic and pharmacologic therapy for mild to moderate dementia." CMAJ, vol. 179, no. 10, pp. 1019-1026, 2008. 


\section{Appendix A}

\section{Cranial Window Surgery Protocol}

\section{Surgical tools}

- Vannas Iridocapsulotomy Scissors

- Jeweler-Type Micro Forceps Curved

- Fine Scissors, Straight, S/S

- Dressing Forceps, Curved, Serr

\section{Procedure}

1. Anesthetize the animal.

2. Remove hair, using an electric shaver and/or hair removal lotion.

3. Apply eye ointment.

4. Secure the animal in the stereotaxic frame.

5. Disinfect the exposed skin using alcohol and betadine.

6. Use the Fine Scissors to remove the skin from the top of the head. Exposing the bregma and lambdoid sutures and an adequate area to the sides of the midsaggital suture for the window to be placed.

7. Use the Dressing Forceps and scissors to remove the fascia from the skull, leaving a dry surface for drilling.

8. Use a dental drill to create a rectangular opening in the skull bone

9. Once the skull is off, always keep aCSF on the brain, applying frequently as it will be absorbed.

10. Use a $30^{1 / 2}$ gauge needle and the Vannas Iridocapsulotomy Scissors to excise the dura mater.

11. Once the dura is removed, place the glass window over the opening (make sure aCSF is added to the exposed brain prior to window placement.

12. Apply super glue to the window, using a $301 / 2$ gauge needle, from glass to bone starting in the center of each side and then filling in.

13. Allow the glue to sit for a few minutes before removing the animal from the stereotaxic frame and placing in an isolated cage. 
Table A.1: Artificial cerebral spinal fluid.

\begin{tabular}{c|ccc}
\hline Solution $^{*}$ & Components & For 1liter $(\mathrm{g})$ & For 20X Stock \\
& $\mathrm{KCl}$ & 0.22 & 4.4 \\
& $\mathrm{MgCl} 2.6 \mathrm{H} 2 \mathrm{O}$ & 0.305 & 6.1 \\
$\mathrm{~A}$ & $\mathrm{CaCl}$ & 0.264 & 5.28 \\
& $\mathrm{NaCl}$ & 7.71 & 154.2 \\
& Urea & 0.402 & 8.04 \\
& Dextrose & 0.665 & 13.3 \\
\hline $\mathrm{B}$ & $\mathrm{NaHCO}$ & 2.066 & 41.32 \\
\hline
\end{tabular}

*- Prepare the solution A and B separately, and mix them together before experiments. The final solution is filtered using $0.22 \mu \mathrm{m}$ filter for perfusion.

**- For 20X stock solution, dilute 20 times and mix together. 


\section{Appendix B}

\section{Immunofluorescence Staining Protocol}

1. 2.5 minutes in $95 \%$ alcohol.

2. Wash in Distilled water.

3. Wash in PBS.

4. Incubate with $0.1 \mathrm{X}$ Triton for 5 minutes.

5. Wash in PBS.

6. Let slides air dry.

7. Use DAP pen to draw a circle around the tissue sections.

8. Wash in PBS $\times 5 \min \times 3$.

9. Incubate with $10 \%$ normal blocking serum in PBS for 20 minutes.

10. Wash in PBS $\times 5 \min \times 3$.

11. Incubate with primary antibody for 60 minutes (in $1.5 \%$ normal blocking serum).

12. Wash in PBS $\times 5$ min $\times 3$.

13. Incubate with secondary antibody for 45 minutes (in 3\% normal blocking serum).

14. Wash in PBS $\times 5$ min $\times 3$.

15. Mount coverslips with aqueous mounting medium or $90 \%$ glycerol in PBS.

16. Examine with fluorescence microscope.

17. Store at room temperature (if mounted with aqueous mounting medium) or $4 \mathrm{C}$ (if mounted with glycerol). 


\section{Vita}

Christy Marie Wilson was born in North Carolina in 1981. She attended Northwood High School in Pittsboro, North Carolina where she graduated in 1999. Following graduation, she studied Computer Science at the University of North Carolina at Charlotte in Charlotte, North Carolina. In 2003, Christy moved to Memphis, Tennessee to study Biomedical Engineering in the University of Tennessee Health Science Center and University of Memphis Joint Program in Biomedical Engineering. She obtained her PhD in Biomedical Engineering and Imaging in May 2009. 